University of Tennessee Health Science Center UTHSC Digital Commons

\title{
THE ROLE OF HSF1 PROTEIN REGULATION ON NEURODEGENERATION
}

\author{
Eunhee Kim \\ University of Tennessee Health Science Center
}

Follow this and additional works at: https://dc.uthsc.edu/dissertations

Part of the Neurosciences Commons

\section{Recommended Citation \\ Kim, Eunhee (http://orcid.org/0000-0002-9297-2848), "THE ROLE OF HSF1 PROTEIN REGULATION ON NEURODEGENERATION" (2017). Theses and Dissertations (ETD). Paper 442. http://dx.doi.org/10.21007/ etd.cghs.2017.0432.}

This Dissertation is brought to you for free and open access by the College of Graduate Health Sciences at UTHSC Digital Commons. It has been accepted for inclusion in Theses and Dissertations (ETD) by an authorized administrator of UTHSC Digital Commons. For more information, please contact jwelch30@uthsc.edu. 


\title{
THE ROLE OF HSF1 PROTEIN REGULATION ON NEURODEGENERATION
}

\begin{abstract}
Cellular protein homeostasis is achieved by a delicate network of molecular chaperones and various proteolytic processes such as ubiquitin-proteasome system (UPS) to avoid a build-up of misfolded protein aggregates. The latter is a common denominator of neurodegeneration. Neurons are found to be particularly vulnerable to toxic stress from aggregation-prone proteins such as $\alpha$-synuclein. Induction of heat-shock proteins (HSPs), such as through activated heat shock transcription factor 1 (HSF1) via Hsp90 inhibition, is being investigated as a therapeutic option for proteinopathic diseases. HSF1 is a master stress-protective transcription factor which activates genes encoding protein chaperones (e.g. iHsp70) and anti-apoptotic proteins. However, whether and how HSF1 is dysregulated during neurodegeneration has not been studied. Here, we discover aberrant HSF1 degradation by aggregated $\alpha$-synuclein (or $\alpha-$ synuclein-induced proteotoxic stress) in transfected neuroblastoma cells. HSF1 dysregulation via $\alpha$ synuclein was confirmed by in vivo assessment of mouse and in situ studies of human specimens with $\alpha$ synucleinopathy. We demonstrate that elevated NEDD4 is implicated as the responsible ubiquitin E3 ligase for HSF1 degradation through UPS. Furthermore, pharmacologically induced SIRT1-mediated deacetylation can attenuate aberrant NEDD4-mediated HSF1 degradation. Indeed, we define the acetylation status of the Lys 80 residue located in the DNA-binding domain of HSF1 as a critical factor in modulating HSF1 protein stability in addition to its previously identified role in the transcriptional activity. Together with the finding that preserving HSF1 can alleviate $\alpha$-synuclein toxicity, the first part of the study strongly suggests that aberrant HSF1 degradation is a key neurodegenerative mechanism underlying $\alpha$ synucleinopathy. Chronic activation of another cellular stress response, unfolded protein response in ER, has been implicated in tauopathy including Alzheimer's disease (AD). The unfolded protein response (UPR) in the endoplasmic reticulum (ER) and the cytoplasmic heat stress response are two major stress response systems necessary for maintaining proteostasis for cellular health. Failure of either of these systems, such as in sustained UPR activation or in insufficient heat shock response activation, can lead to the development of neurodegeneration. Alleviation of ER stress and enhancement of heat shock response through heat shock factor 1 (HSF1) activation have previously been considered as attractive potential therapeutic targets for AD-a prevalent and devastating tauopathy. The second part of the study concentrates on our attempts to understand the interplay of the two aforementioned systems and their cooperative role in AD. We provide compelling in vitro and in vivo evidence that strongly suggests an autopropagating interplay of UPR activation and HSF1 degradation being a common pathogenic feature in both human $A D$ and tau transgenic mouse $A D$ models. We identify aging-associated $A D$-like neuropathological changes in the hippocampus of HSF1 heterozygous knock-out mice. We speculate that HSF1 loss as an early (earliest) event which constitutes a mechanistic connection between ER stress and tau hyperphosphorylation in tau pathology. Finally, we demonstrate that aged mice lacking HSF1 gene exhibited deficits in hippocampal-dependent functions including short-term working memory, spatial learning and long-term memory. All together, our work supports a previously underappreciated importance of this master stress regulator HSF1 in neuronal functions and in maintaining brain homeostasis.
\end{abstract}

\section{Document Type}

Dissertation

Degree Name

Doctor of Philosophy (PhD)

Program

Biomedical Sciences 


\section{Research Advisor}

Francesca-Fang Liao, Ph.D.

\section{Keywords}

Neurodegenerative diseases, Protein degradation, Proteinopathies, Stress response

\section{Subject Categories}

Medicine and Health Sciences | Neurosciences 


\title{
THE ROLE OF HSF1 PROTEIN REGULATION ON NEURODEGENERATION
}

\author{
A Dissertation \\ Presented for \\ The Graduate Studies Council \\ The University of Tennessee \\ Health Science Center
}

In Partial Fulfillment

Of the Requirements for the Degree

Doctor of Philosophy

From The University of Tennessee

By

Eunhee Kim

May 2017 
Copyright (C) 2017 by Eunhee Kim. All rights reserved. 


\section{DEDICATION}

This dissertation is dedicated to my parents, my brother and friends for their love and support. 


\section{ACKNOWLEDGEMENTS}

I would like to give my deepest gratitude to my academic advisor, Dr. FrancescaFang Liao for accepting me into her group and her excellent guidance and training. She continuously showed wholehearted genuine support by providing not only all the valuable internal and external collaborative environment but also intellectual freedom in my work. I would like to thank my committee members, Dr. Edwards Park, Dr. Michael McDonald, Dr. Junmin Peng, and Dr. Ioannis Dragatsis for their generous guidance and encouraging support to pursue my research goals.

I would like to give my special thanks to my former and present labmates, especially, Ruishan Wang, Jingjing Li, Bin Wang, and Lubin Lan who have always been kind and supportive like family.

I would like to express my gratitude to Dr. Peter Nelson at University of Kentucky for enabling studies on human specimens which highly improved this thesis work. I am grateful to Dr. Dennis Thiele at Duke University who inspired me with his research and showed his interest in my work, sharing his scientific expertise during our collaboration.

I would like to acknowledge The Neuroscience Institute at UTHSC whom has provided an excellent professional development environment and resources for completion of research projects.

I would like to thank my classmates, Cameron Ogg and Nick Saites for their kindness during the coursework. Especially, I am very lucky to have met Taeyeon Kim and Andrea Balogh, and I thank them for being wonderful friends who are reliable and provide me with clear guidance whenever I am caught in difficult situations. First and foremost, I would like to thank my beloved family and friends who spiritually supported me during my time in Memphis. 


\begin{abstract}
Cellular protein homeostasis is achieved by a delicate network of molecular chaperones and various proteolytic processes such as ubiquitin-proteasome system (UPS) to avoid a build-up of misfolded protein aggregates. The latter is a common denominator of neurodegeneration. Neurons are found to be particularly vulnerable to toxic stress from aggregation-prone proteins such as $\alpha$-synuclein. Induction of heat-shock proteins (HSPs), such as through activated heat shock transcription factor 1 (HSF1) via Hsp90 inhibition, is being investigated as a therapeutic option for proteinopathic diseases. HSF1 is a master stress-protective transcription factor which activates genes encoding protein chaperones (e.g. iHsp70) and anti-apoptotic proteins. However, whether and how HSF1 is dysregulated during neurodegeneration has not been studied. Here, we discover aberrant HSF 1 degradation by aggregated $\alpha$-synuclein (or $\alpha$-synuclein-induced proteotoxic stress) in transfected neuroblastoma cells. HSF1 dysregulation via $\alpha$-synuclein was confirmed by in vivo assessment of mouse and in situ studies of human specimens with $\alpha-$ synucleinopathy. We demonstrate that elevated NEDD4 is implicated as the responsible ubiquitin E3 ligase for HSF1 degradation through UPS. Furthermore, pharmacologically induced SIRT1-mediated deacetylation can attenuate aberrant NEDD4-mediated HSF1 degradation. Indeed, we define the acetylation status of the Lys 80 residue located in the DNA-binding domain of HSF1 as a critical factor in modulating HSF1 protein stability in addition to its previously identified role in the transcriptional activity. Together with the finding that preserving HSF1 can alleviate $\alpha$-synuclein toxicity, the first part of the study strongly suggests that aberrant HSF1 degradation is a key neurodegenerative mechanism underlying $\alpha$-synucleinopathy.
\end{abstract}

Chronic activation of another cellular stress response, unfolded protein response in ER, has been implicated in tauopathy including Alzheimer's disease (AD). The unfolded protein response (UPR) in the endoplasmic reticulum (ER) and the cytoplasmic heat stress response are two major stress response systems necessary for maintaining proteostasis for cellular health. Failure of either of these systems, such as in sustained UPR activation or in insufficient heat shock response activation, can lead to the development of neurodegeneration. Alleviation of ER stress and enhancement of heat shock response through heat shock factor 1 (HSF1) activation have previously been considered as attractive potential therapeutic targets for $\mathrm{AD}$ - a prevalent and devastating tauopathy. The second part of the study concentrates on our attempts to understand the interplay of the two aforementioned systems and their cooperative role in AD. We provide compelling in vitro and in vivo evidence that strongly suggests an autopropagating interplay of UPR activation and HSF1 degradation being a common pathogenic feature in both human $\mathrm{AD}$ and tau transgenic mouse AD models. We identify aging-associated AD-like neuropathological changes in the hippocampus of HSF1 heterozygous knock-out mice. We speculate that HSF1 loss as an early (earliest) event which constitutes a mechanistic connection between ER stress and tau hyperphosphorylation in tau pathology. Finally, we demonstrate that aged mice lacking HSF1 gene exhibited deficits in hippocampal-dependent functions including short-term working memory, spatial learning and long-term memory. All together, our work 
supports a previously underappreciated importance of this master stress regulator HSF1 in neuronal functions and in maintaining brain homeostasis. 


\section{TABLE OF CONTENTS}

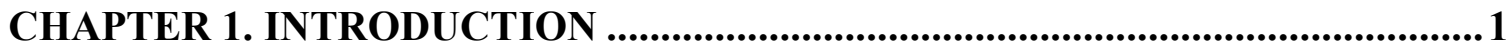

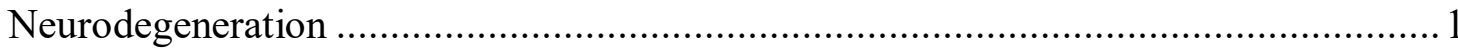

The Two Most Common Neurodegenerative Diseases: Alzheimer's Disease and

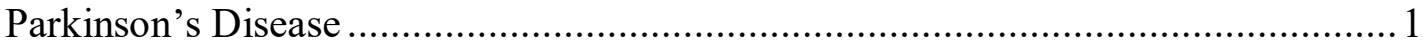

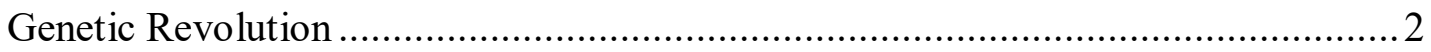

Protein Aggregates in Neurodegenerative Diseases: Proteinopathy .........................

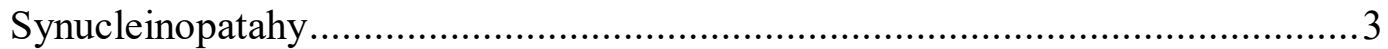

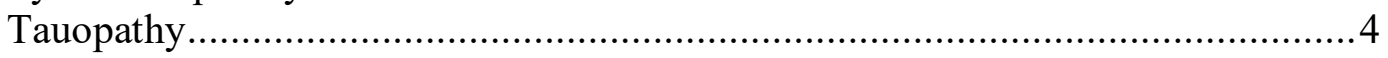

Protein Quality Control and Stress in Neurodegeneration ........................................ 7

Cellular Stress Response (Heat Shock Response) ……...................................... 8

Ubiquitin Proteasome System and Neurodegeneration ........................................ 8

Cellular Stress Response in ER and Neurodegeneration: Unfolded Protein

Response (UPR) as a Result of ER Stress......................................................... 9

Autophagy-Lysosomal Pathway and Neurodegeneration........................................ 11

Heat Shock Factor 1 Regulation and Neurodegeneration ........................................ 12

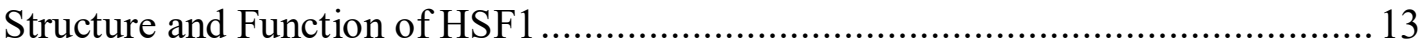

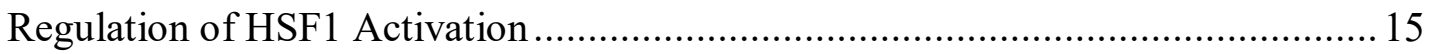

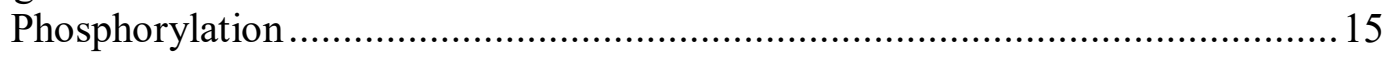

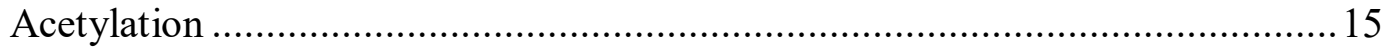

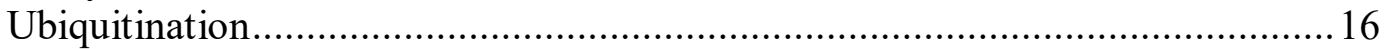

Heat Shock Proteins and Neurodegeneration....................................................... 16

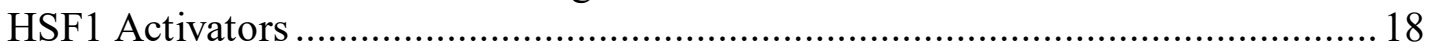

Aging-related Changes in HSF1 and SIRT1 Activity ……................................. 18

Hypothesis, Rationale, and Specific Aims ......................................................... 20

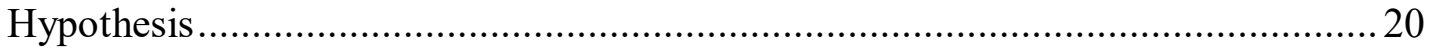

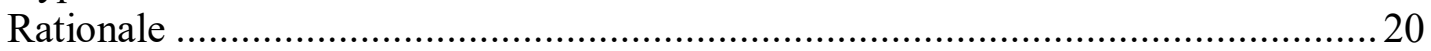

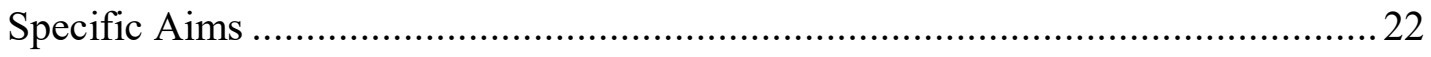

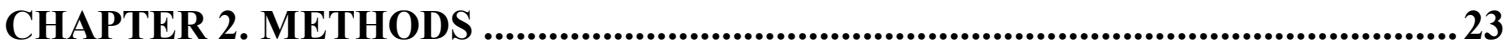

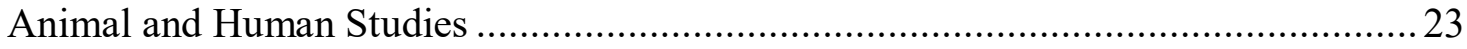

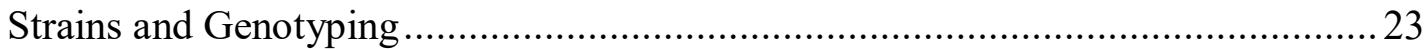

Intraperitoneal Injection and Intra Substantial Nigra Viral Microinjection..............23

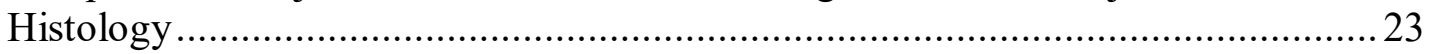

Synaptosomal Membrane Preparation and Sarkosyl Insolubility Assay ...................22

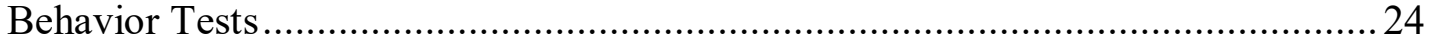

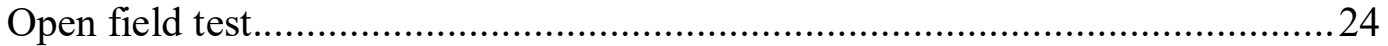

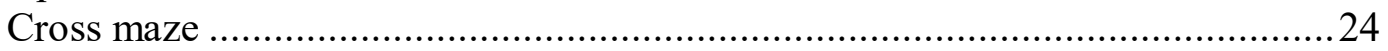

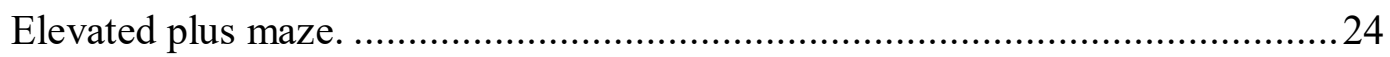

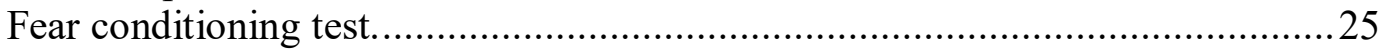

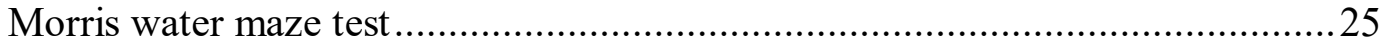

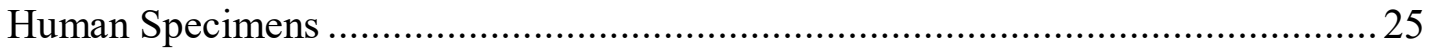

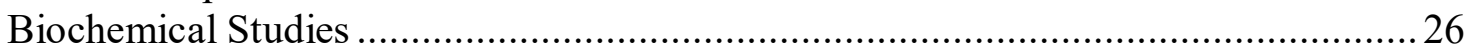

Cell Cultures and Generation of Stable Cell Lines................................................26 


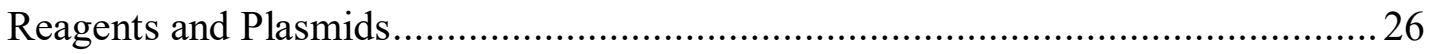

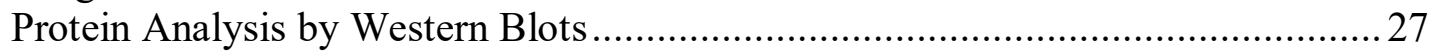

In Vivo Ubiquitination Assay and Cycloheximide Chase Assay ...........................22

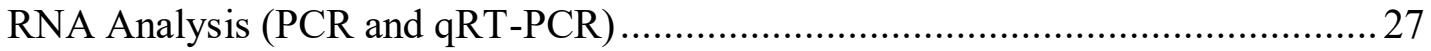

MTT (3-(4,5-dimethylthiazol-2-yl)-2,5-diphenyltetrazolium bromide) Assay.........22

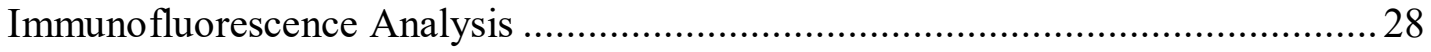

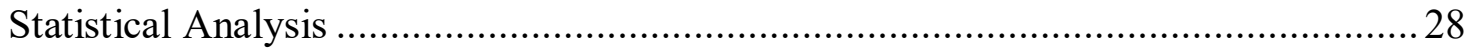

\section{CHAPTER 3. THE ROLE OF HSF1 PROTEIN REGULATION IN}

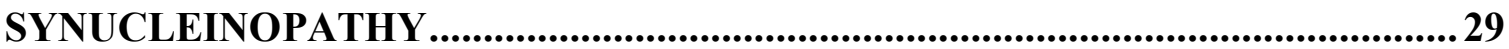

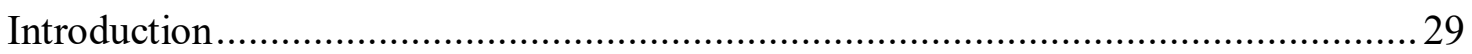

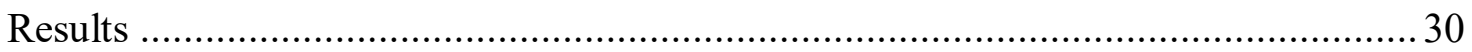

Overexpressed $\alpha$-syn Protein Promotes Ubiquitination and Degradation of HSF1

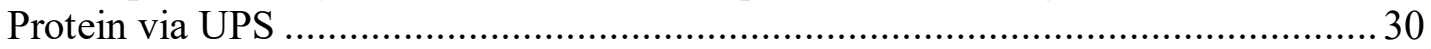

$\alpha$-Syn-induced HSF1 Degradation is Mediated by the E3 ligase NEDD4 ................ 30

Loss of HSF1 Protein Induced by $\alpha$-synucleinopathy in vivo ……………............... 35

The Role of Acetylation Status in NEDD4-mediated HSF1 Degradation via

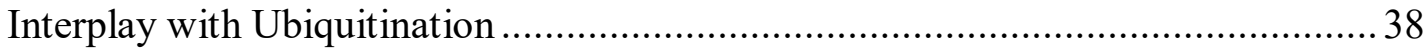

Downregulation of NEDD4 or Overexpression of HSF1 or RSV Provides

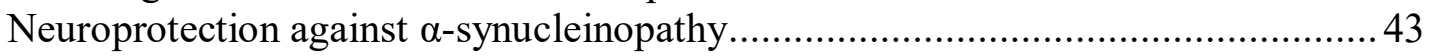

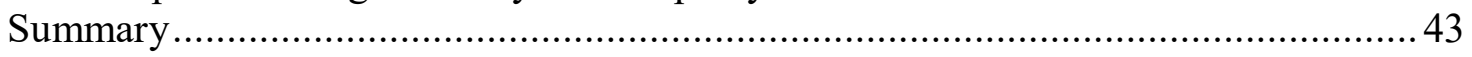

\section{CHAPTER 4. THE ROLE OF HSF1 PROTEIN REGULATION IN}

TAUOPATHY

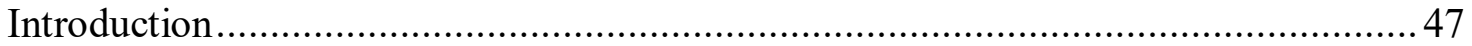

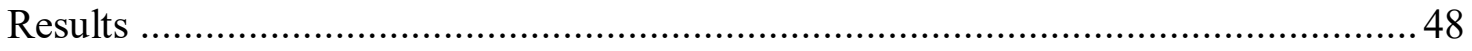

Loss of HSF1 Protein Is an Early Event That Precedes NFTs Formation in AD,

Which Is Further Exacerbated in the Presence of PERK (or UPR) Activation in the Brains of Mouse and Human Tauopathy ........................................................ 48

Haploinsufficiency of HSF1 Leads to Chronic PERK Activation and Causes

Aberrantly Phosphorylated Tau Aggregation and Its Mislocalization in the Aged

Mouse Hippocampus ……................................................................................5 50

eIF2 $\alpha$-CHOP Activation Is Involved in Autophagy-Lysosomal HSF1 Protein

Degradation during Chemically Induced ER Stress in Neurons ...............................56

Overexpressed Pro-Aggregation Mutant Tau ${ }_{R D} \Delta$ K280 Leads to HSF1

Degradation Which Is Further Exacerbated by eIF2 $\alpha$-CHOP Activation ..................56

Aberrant HSF1 Degradation and HSP70 a5 (BiP/GRP78) Attenuation Are

Associated with Tau Accumulation and Toxicity in Tauopathy ..............................58

Accumulation of Both $\alpha$-syn and Tau and Increased Activity of Tau Kinases

Were Detected in the Brainstem of HSF1-haploinsufficient Mice ……………........63 63

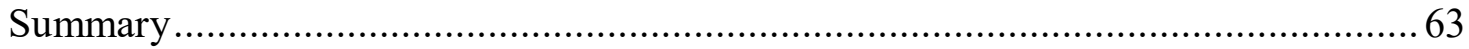

\section{CHAPTER 5. BEHAVIORAL CHARACTERIZATION OF HSF1

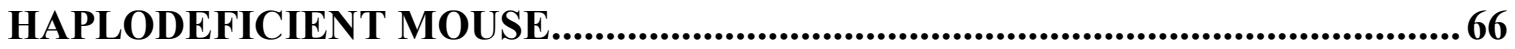

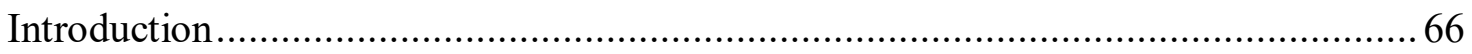




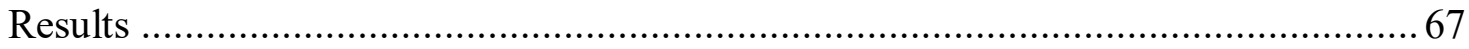

Aged HSF1-haploinsufficient Mice Exhibit Increased Anxiety Levels in the Elevated Plus Maze, Not in the Open Field Test

Aged HSF1-haploinsufficient Mice Exhibit Hippocampal-Dependent Learning

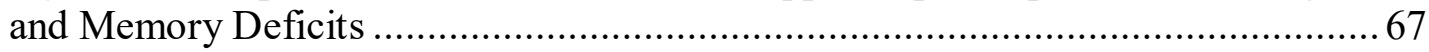

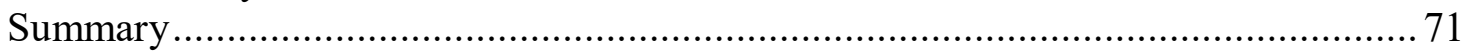

CHAPTER 6. DISCUSSIONS AND FURTHER DIRECTIONS ...............................75

Aberrant HSF1 Degradation in Synucleinopathy ………........................................ 75

HSF1 Degradation as a Mechanistic Link between UPR Activation and Tau

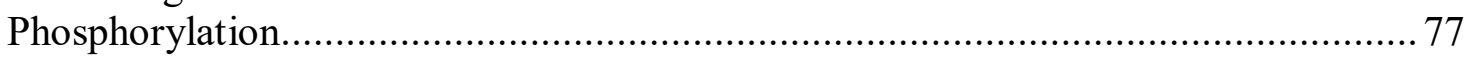

Synaptic Tau Pathology and HSF1 Dysregulation..................................................... 80

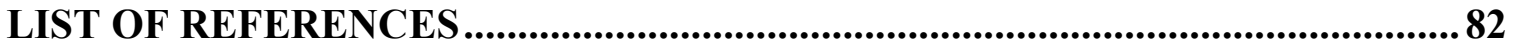

VITA 


\section{LIST OF FIGURES}

Figure 1-1. HSF1 Regulation by Post-Translational Modifications. ........................... 14

Figure 1-2. HSF1 Activation by an Hsp90 Inhibitor............................................... 19

Figure 1-3. Aberrant HSF1 Degradation as a Common and Important Key Molecular Mechanism Underlying Neurodegeneration......................................... 21

Figure 3-1. $\quad \alpha-$ Syn Aggregation Causes HSF1 Degradation via UPS......................... 31

Figure 3-2. NEDD4 Promotes Ubiquitination and Proteasomal Degradation of HSF1 in Neuroblastoma cells Overexpressing A53T $\alpha$-syn............................ 33

Figure 3-3. Reciprocal NEDD4 and HSF1 Levels in Mouse and Human Tissue of $\alpha$ -

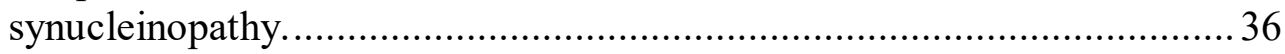

Figure 3-4. HSF1 Degradation Induced by AAV- $\alpha$-syn Viruses in Mouse Substantia Nigra.

Figure 3-5. HSF1 Acetylation/Deacetylation and Ubiquitination.

Figure 3-6. Effects of Down-regulated NEDD4 or Overexpressed HSF1 or RSV on $\alpha$-syn Toxicity.

Figure 4-1. HSF1 Protein Loss and Chronic UPR Activation in the Brain of rTg(tauP301L)4510, and Minor Loss of HSF1 Protein in the Brain of PS19 (tauP301S).

Figure 4-2. HSF1 Loss and UPR Activation in the Frontal Cortex of Human Brain at Different Stages of Alzheimer's Disease Progression (Braak Staging)...... 51

Figure 4-3. Chronic UPR Activation and Aberrantly Hyperphosphorylated Tau Aggregation in the Hippocampus of Aged HSF1 Haploinsufficient Mouse.

Figure 4-4. A Graph Shows Quantitative Measurement of the Relative Tau Phosphorylation Normalized to Total Tau (Tau46) in the Hippocampus of WT and HSF1+/- at 2, 6, and 9 Months of Age.

Figure 4-5. Double Staining with Thioflavin S (green, a) and Tau46 Antibody (red,

b) in the Hippocampus of 13 Month-old HSF1 +/-

Figure 4-6. Abnormal Mislocalization of Hyperphosphorylated Tau in the Hippocampus of HSF1 Haploinsufficient Mouse. 
Figure 4-7. Autophagy-Lysosomal HSF1 Protein Degradation Mediated by eIF2 $\alpha$ CHOP Activation during Chemically Induced Acute ER Stress in Neurons.

Figure 4-8. HSF1 Degradation and CHOP Activation in N2a-TauRD $\Delta$ K280 Stable Cell Line.

Figure 4-9. HSF1 Degradation Caused by Overexpressed Tau $\mathrm{RD}_{\Delta \mathrm{K}} \mathrm{K} 280$ and Further Facilitated by eIF2 $\alpha$-CHOP Activation. 60

Figure 4-10. The Involvement of Aberrant HSF1 Degradation and HSP70 a5 (BiP/GRP78) Attenuation in Tau Accumulation and Toxicity in Tauopathy.

Figure 4-11. Suppressed Induction of HSP70 a5 (BiP/GRP78) upon Chemical ER Stress in $\mathrm{N} 2 \mathrm{a}-\mathrm{Tau}_{\mathrm{RD}} \Delta \mathrm{K} 280$.

Figure 4-12. Accumulation of Both $\alpha$-syn and Tau and Increased Activity of Tau Kinases Were Detected in the Brainstem of HSF1+/-

Figure 5-1. Locomoter Activity and Explorative Behavior in the Open Field Test.

Figure 5-2. Increased Anxiety-related Behavior in HSF1-haploinsufficient Mice in the Elevated Plus Maze.

Figure 5-3. Impaired Working Memory in HSF1-haploinsufficient Mice in the Cross Maze.

Figure 5-4. Fear Acquisition and Contextual Fear Memory in the Contextual Fear Conditioning Test.

Figure 5-5. Defective Spatial Learning and Memory in HSF1-haploinsufficient Mice in the Water Maze.

Figure 6-1. Hypothetic Model on a Vicious Cycle of UPR Activation and HSF1 Degradation in Tauopathy. .78 


\section{LIST OF ABBREVIATIONS}

17-AAG

ALS

AMPK

APP

ATF6

$\alpha$-syn

BDNF

CaMKII

CDK5

CHIP

CHOP

CMA

CNS

DLB

DMSO

ER

ERK1

FBXW7

FTD

GSK3

HD

HSC70 17-allylamino-17-desmethoxygeldanamycin

Amyotrophic Lateral Sclerosis

5' AMP-activated protein kinase

Amyloid precursor protein

Activating transcription factor 6

$\alpha$-synuclein

Brain-derived neurotrophic factor

Ca2+/calmodulin-dependent protein kinase II

Cyclin-dependent kinase 5

C-terminus of Hsc 70-interacting protein

The transcription factor C/EBP homologous protein

Chaperone-mediated autophagy

Central nervous system

Dementia with Lewy Bodies

Dimethyl sulfoxide

Endoplasmic reticulum

Extracellular signal-regulated protein kinases 1

F-box/WD repeat-containing protein 7

Frontotemporal dementia

Glycogen synthase kinase 3

Huntington's disease

Heat shock cognate 70 


\begin{tabular}{|c|c|}
\hline HSE & Heat shock element \\
\hline HSF1 & Heat shock factor 1 \\
\hline HSP90 & Heat shock protein 90 \\
\hline HSPs & Heat shock proteins \\
\hline IRE1 & Inositol-requiring enzyme 1 \\
\hline JNK & c-Jun N-terminal kinase \\
\hline LAMP2A (or 1) & Lysosome-associated membrane glycoprotein 2A (or 1) \\
\hline LC3 & Microtubule-associated protein 1A/1B-light chain 3 \\
\hline LRRK2 & Leucine Rich Repeat Kinase 2 \\
\hline MAPK & Mitogen-activated protein kinase \\
\hline mTOR & The mechanistic target of rapamycin \\
\hline NMDA & N-Methyl-D-aspartic acid \\
\hline NFTs & Neurofibrillary tangles \\
\hline PD & Parkinson's disease \\
\hline PERK & Protein kinase $\mathrm{R}$ (PKR)-like endoplasmic reticulum kinase \\
\hline PI3K & Phosphoinositide 3-kinase \\
\hline PS & Presenilin \\
\hline TDP-43 & Transactive response DNA binding protein $43 \mathrm{kD}$ \\
\hline UPR & Unfolded protein response \\
\hline UPS & Ubiquitin proteasome system \\
\hline VCP & Valosin-containing protein \\
\hline
\end{tabular}




\section{CHAPTER 1. INTRODUCTION}

\section{Neurodegeneration}

Neurodegenerative diseases are chronic and progressive disorders characterized by selective and gradual neuronal loss in cognitive or motor systems in the brain. Such diseases are incurable fetal diseases. The etiology and pathogenesis of neurodegenerative disorders remain largely unknown. Characterization of disease specific markers and the patterns of neuronal loss enabled us to classify neurodegenerative disorders. Specific disorders include Alzheimer's disease (AD), Parkinson's disease (PD), Huntington's Disease (HD), Amyotrophic Lateral Sclerosis (ALS), Frontotemporal Dementia (FTD), Motor neurone diseases, Spinocerebellar ataxia, and Spinal muscular atrophy.

\section{The Two Most Common Neurodegenerative Diseases: Alzheimer's Disease and Parkinson's Disease}

$\mathrm{AD}$ known as the sixth leading cause of death in the United States is the only disease among the top ten causes of death that currently has no prevention and cure. By 2050, the number of people aged 65 and older suffering from AD is estimated to triple from 5.2 million in 2016 to 13.8 million (Alzheimers association, 2016). AD was first described in 1906 by Dr. Alois Alzheimer. The hippocampus is the primary severely affected area in AD undergoing drastic shrinkage due to neuronal loss. Numerous animal studies suggest that an intact hippocampus is critical for learning and memory. The most common initial symptom of AD is to have difficulty in acquiring new information. Symptoms at the fully developed final stage include impairment of cognitive and memory function, as well as deformed personality. Acetylcholinesterase inhibitors and NMDA receptor antagonists are the two FDA-approved drugs showing small benefits for treating cognitive problems.

$\mathrm{PD}$ is the second most prevalent neurodegenerative disease after AD. An estimated 1.5 million people in the United States alone have PD. PD was first described in 1817 by James Parkinson. The symptoms of PD include motor (e.g., treamor, bradykinesia, rigidity, postural instability) and non-motor dysfunction (cognitive impairment, depression, sensory dysfunction). The motor symptoms of PD are caused by the selective loss of neurons in the substatia nigra that produce dopamine (a neurotransmitter that helps purposeful movement), leading todepletion of dopamine in the striatum (Davie et al., 2008). L-DOPA, a direct precursor to dopamine, and dopamine agonists that have similar effects as L-DOPA can diminish (or alleviate) motor symptoms to large degrees. Monoamine oxidase inhibitors cause increased levels of L-DOPA in the striatum and therefore improve motor symptoms as well. 


\section{Genetic Revolution}

The identification of mutations in several genes has advanced our understanding of the pathogenesis of neurodegenerative diseases. HD is a hereditary neurodegenerative disorder of mid-life onset caused by aberrantly long poly-glutamine sequence as a result of expansion of CAG triplet repeats in the huntingtin gene on chromosome 4. HD is characterized by progressive cognitive decline and chorea. With the exceptions of polyglutamine diseases including HD, Spinocerebellar ataxia, and Spinal muscular atrophy, many other neurodegenerative diseases are predominantly sporadic in nature which cases involve multiple genetic and environmental factors playing key roles in their pathogenesis.

Most PD cases are sporadic and only less than $10 \%$ of patients are associated with familial forms of PD. Whereas mutations in SNCA and LRRK2 genes cause autosomal-dominant PD forms, mutations in Parkin, PINK, DJ-1 and ATP13A2 genes are responsible for autosomal-recessive forms of PD. Among these mutations, SNCA, the first gene identified in PD, encodes $\alpha$-synuclein, abundant cytosolic protein with 140 amino acids. Mutations in LRRK2 are found to be the most common genetic cause of both familiar and sporadic cases of PD. AD can be grouped into four subcategories by two major criterias, early onset or late-onset and sporadic or familial. People diagnosed in thirties, fourties, and fifties fall into early-onset disease (EOD). It is estimated that less than $5 \%$ of all AD cases are EOD. The majority of AD cases are sporadic and only less than $1 \%$ of all AD cases fall into familial AD (FAD). While the symptoms of early-onset FAD and late-onset AD (LOAD) appear to be similar to each other, they may have different underlying pathogenic mechanisms. Neurogenetists discovered from their studies on early-onset FAD that there are three distinct genes that cause familial AD. They are APP on chromosome 21, presenilin-1 on chromosome 14, and presenilin-2 on chromosome 1. Although FAD is rare, the discovery of genetic mutations has made it possible to identify and understand the pathogenic mechanism of $A D$. In addition to FAD mutations, there is a risk factor first identified with its great impact on $\mathrm{AD}$, a E4 genetic variant of a gene located on chromosome 17 called Apolipoprotein E-e4 (APOE4). APOE4 was first discovered in 1993 to be a genetic variant that increases the chance of developing AD. Sporadic AD with unknown cause is considered to be largely attributable to gradual accumulation of aging-associated malfunctioning in cellular system.

\section{Protein Aggregates in Neurodegenerative Diseases: Proteinopathy}

Proteinopathy refers to diseases caused by defects in protein synthesis, folding, trafficking, or degradation in cells. Protein aggregation is a common hallmark of neurodegeneration. Immunohistochemical staining of postmortem human brain, which was first developed in 1980s, has enabled us to understand cellular changes in disease states. One important discovery made by immunohistochemistry is the identification of protein aggregates in neurodegenerative diseases. Intraneuronal aggregates such as huntingtin, synuclein, and TDP-43 are found in PD, HD, and ALS, respectively, whereas $\mathrm{AD}$ is known for having both intra- and extracellular aggregates. The first purification of 
neurofibrillary tangles (NFTs) and amyloid beta (A $\beta$ ) in the brains of people suffering from AD was described by Ihara et al., 1986 and Glenner and Wong, 1984, respectively. As these proteins became the focus of $\mathrm{AD}$ research field, there were two groups of scientists who each claimed that either tau or $A \beta$ is more likely to cause AD. Now there is growing evidence that tau protein may be a key driver of AD pathogenesis (Roberson et al., 2007, Morris et al., 2011). Roberson and colleagues crossed A $\beta$-forming APP transgenic mice with Tau knock-out mice. Tau reduction prevented behavioral deficits and premature mortality in APP transgenic mice. In addition, they demonstrated that tau reduction increased resistance to excitotoxin-induced seizures.

As of the progressive characteristic of $\mathrm{AD}$, plaques and tangles gradually spread throughout the brain via synapses. It has been highlighted that proteinopathy may underlie the prime mechanism responsible for the spread of neurodegeneration. In order to appropriately target the toxic species, which forms of $\mathrm{A} \beta$ and tau are synaptotoxic species needs to be determined. The relative role of soluble protein versus insoluble aggregates needs to be accurately identified. Whether protein aggregates themselves are pathogenic or as incidental or beneficial response still remains as an open question in the field.

Synucleinopatahy. Monomeric $\alpha$-synuclein ( $\alpha$-syn) is intrinsically disordered protein that lacks a fixed stable structure with an approximate size of $14 \mathrm{kDa}$. This protein can acquire $\alpha$-helical secondary structure only upon binding to lipid vesicles. $\alpha$ syn mainly found in presynaptic terminals can interact with synaptic vesicles by binding to membrane via its $\mathrm{N}$-terminal domain consisting of seven of the 11-residue repeats (Jensen et al., 1998, McLean et al., 2000). The physiological function of $\alpha$-syn was demonstrated in $\alpha$-syn knock out mice to be involved in synaptic plasticity and synaptic vesicle homeostatsis (Cabin et al., 2002). Mice lacking $\alpha$-syn gene showed impaired spatial learning and memory (Kokhan et al., 2012). The aggregation propensity of $\alpha$-syn can be accelerated by three point mutations of human $\alpha$-syn, A53T, A30P, and E46K, that are known to be autosomal dominant and linked to early-onset PD (Polymeropoulos et al., 1997). These mutations are located in the $\mathrm{N}$-terminal repeats to which synaptic vesicles bind and suppress $\alpha$-syn binding to vesicles, leading to loss of $\alpha$-syn function (Jensen et al., 1998). $\alpha$-syn purified from mammalian cells exists as a stable helical tetramer with an apparent size of 58-60 kDa (Bartels et al., 2011). Various forms of physiological $\alpha$-syn (60-, 80-, 100- kDa multimers) were later found to exist in freshly biopsied normal human brain specimen (Dettmer et al., 2015). Of note, tetrameric $\alpha$-syn is more likely to resist aggregation than unfolded $\alpha$-syn monomer (Bartels et al., 2011, Dettmer et al., 2015). It turned out that expression of $\alpha$-syn multimers was decreased in hA53T mice and A53T iPSC neurons (Dettmer et al., 2015). As a result, $\alpha$-syn aggregation is expected to produce neurotoxicity through a gain of function mechanism.

Synucleinopathy refers to a class of diseases associated with abnormal deposition of $\alpha$-syn protein. Synucleinopathy is a major class of neurodegenerative diseases including PD, dementia with Lewy bodies (DLB), and multiple system atrophy. In synuclenopathy, $\alpha$-syn is found to abnormally polymerize into fibrils, forming the 
patholgocial inclusions such as Lewy body, and Lewy neurites. Lewy body represents intracellular spherical masses composed of not only $\alpha$-syn but also other proteins such as ubiquitin and neurofilament protein. Whereas PD primarily affects dopaminergic neurons in the midbrain, Diffuse Lewy body disease, also called Lewy body dementia, has Lewy body inclusions mainly in cortical neurons.

There is a hypothesis devised to explain the regional patterns of synuclein pathology, which is called Braak staging of PD. Based on the varying degrees of synuclein pathology, Braak and his group proposed a temporal sequence of events occurring in PD brain (Braak et al., 2002). In stage 1, synuclein pathology is limited to medulla oblongata or the olfactory bulb. In stage 2, rostrally adjacent structures including the caudal raphe nuclei and the locus cerulues are additionally included. Stage 3 includes the brain regions of amygdala and substantia nigra. Neuronal loss accompanied with synuclein pathology starts to be detected in substantia nigra from stage 3 or 4 . However, a pitfall in the Braak staging scheme is that it cannot define where PD begins. Patients with LRRK2 mutation, an autosomal-dominant PD mutation, do not display Lewy body pathology except for the cases of G2019S LRRK mutation (Burke et al., 2008). In addition, it is not tenable to assume that the presence of synuclein deposition necessarily correlates with neuronal death. Rather, an inverse relationship between aggregate formation and neuronal toxicity was demonstrated in human PD brain, Drosophila PD model and in vitro HD model (Arrasate et al., 2004, Chen et al., 2005). Taken together, it should be considered that Lewy body solely may not be a reliable indicator of cellular dysfunction manifested in PD.

Tauopathy. Tau, mostly located in axons of neurons, is a microtubule binding protein responsible for microtubule assembly and axonal transport in neurons. The MAPT gene in chromosome 17q21 encodes tau protein. As a result of alternative splicing in exons 2, 3, and 10 of the MAPT (microtubule-associated protein tau) gene, six isoforms of tau have been identified in human brain, ranging in size from 352 to 441 amino acids. The N-terminal inserts are coded by exons 2 and 3, resulting in tau isoforms with $0 \mathrm{~N}, 1 \mathrm{~N}$, and $2 \mathrm{~N}$. Each exon of 9, 10, 11, and 12 encodes a microtubule binding motif, and the existence of exon 10 is a determining factor between the three $3 \mathrm{R}$ ( 3 repeated domains) tau isoforms and the other three $4 \mathrm{R}$ (4 repeated domains) tau isoforms. The binding ability of $4 \mathrm{R}$ tau to microtubules is stronger than that of $3 \mathrm{R}$ tau. $3 \mathrm{R}$ and $4 \mathrm{R}$ tau contribute almost equally to total human tau. 1N3R and 1N4R are known to be the most abundant forms in human brain. In $\mathrm{AD}$, increase in $4 \mathrm{R}$ and/or decrease in $3 \mathrm{R}$ results in approximately 2:1 4R:3R ratio. Considering 45 serine, 35 threonine, and 5 tyrosine residues in the total 441 residues of tau protein, tau phosphorylation is a very complex process. Regulation of tau isoforms and tau phosphorylation are the two major mechanisms of controlling the proper functioning of tau protein. In contrast to physiological forms of tau, pathologically hyperphosphroylated tau called paired helical filament (PHF)-tau is dissociated from axon and forms insoluble fibrillary inclusions called neurofibirallray tangles (NFTs) that highly accumulate in neuronal soma and dendrites, leading to neuronal degeneration. Change in tau distribution from axonal to dendritic spine is one early key event in AD brain (Kosik et al., 1989). 
Tauopathy is characterized by a build-up of tau aggregates which underlies the pathogenic mechanisms of a number of neurodegenerative diseases. Tauopathy is not limited to AD pathology. Tau protein is frequently found in $\alpha$-syn positive inclusion (Arima et al., 1999, Ishizawa et al., 2003). Tau and $\alpha$-syn that co-exist in various pathological conditions appear to accelerate the fibrillization of each other in vitro and in vivo (Kotzbauer et al., 2004, Moussaud et al., 2014). Tauopathy includes Frontotemporal dementia (FTD), the second or third most common type of dementia that accounts for less than $5 \%$ of all dementia. Approximately 50-70\% cases of FTD are sporadic, and 20$40 \%$ cases account for familial FTD. Only $10 \%$ cases are inherited FTD with a clear familial history of disease being passed down from the parents. The primary genes linked to FTD are tau and progranulin. Around $30 \%$ of inherited FTD are caused by mutations in the tau gene. Other rare genetic causes of FTD include transactive response DNA binding protein (TDP), valosin-containing protein, FUS, and sequestosome 1. Frontotemporal lobar degeneration (FTLD) is a pathological term for FTD. Depending on the feature of protein aggregates, it can further be categorized into FTLD-Tau, FTLDTDP (or FTLD-U), and FTLD-FUS. Pick's disease is one type of FTD syndrome and a rare form of progressive dementia that only affects certain areas of the brain, such as frontal and temporal lobe, distinct from $\mathrm{AD}$ that progressively and broadly targets the brain. Whereas memory loss is the earliest and primary symptom of $\mathrm{AD}$, personality change is an early symptomatic characteristic of Pick's disease. Tau deposits found in pick's disease are mostly composed of 3R tau.

Frontotemporal dementia and Parkinsonism linked to chromosome 17 (FTDP-17) is an autosomal dominant neurodegenerative disease that likely accounts for only a small percentage of FTD. The symptoms of this disorder include behavioral and personality disturbances, cognitive deficits, and motor dysfunction. The name of the disease is coined for mutations in the tau gene located in chromosome 17 that are found in human FTDP17 patients. It is a very rare disease that 38 tau genetic mutations have been identified over 100 families. The mutations found in the tau gene are either coding site or intronic mutations. Intronic mutations largely located in intron between exon 10 and 11 influence the exclusion (or inclusion) of exon 10, affecting the ratio of 4R- to 3R tau. About $90 \%$ of coding mutations with missense or deletion changes are located in the $\mathrm{C}$-terminal end of tau, altering the function of tau. Some mutations located in exon 10 likely lead to the proportional increase of $4 \mathrm{R}$ to $3 \mathrm{R}$ tau. $\mathrm{P} 301 \mathrm{~L}$ mutation in exon 10 is the most prevalent mutant form found in FTDP-17 patients.

Despite the absence of tau mutation that causes familial AD, our understanding of tau pathogenesis has been advanced by identification of tau mutations in FTDP-17 patieints. Based on FTDP-17 mutations, several tau pathogenic mouse models have been genetically engineered and characterized in recent years. The presence of NFTs in these mouse models has enabled us to identify mechanisms by which pathogenic tau contributes to $\mathrm{AD}$ progression. The difference in the tau gene between murine and human is that murine tau only has $4 \mathrm{R}$ domain, and there are 14 amino acids differring in the $\mathrm{N}$ terminal domain between them. Two decades ago, a tau transgenic mouse expressing the longest human tau isoform was first generated and reported to exhibit mis-localized and hyperphosphorylated tau protein (Götz et al., 1995). Human tau protein expressed in this 
mouse model was considered as pre-tangle form, not NFTs, as they did not form any insoluble tau aggregates. The limitation of such transgenic mouse ooverexpressing only one isoform was overcome by Duff et al., 2000. They generated tau transgenic mouse model expressing all six isoforms of human tau transgenes acquired from the human P1derived artificial chromosome. Three years later, the same group circumvented any potential effect of endogenous murine tau by establishing human tau gene overexpression in the tau knock-out mice. These mice developed somatic accumulation of phosphorylated tau by 3 months of age, and at 6 months of age, inclusions thought to be NFTs were detected (Andorfer et al., 2003).

Later, Santacruz et al., 2005 created rTg4510 transgenic mouse model that overexpresses mutant tau P301L gene $(4 \mathrm{R} / 0 \mathrm{~N})$ which is constitutively expressed until inactivated by doxycycline administration. Pre-tangles began to form as early as 2.5 months. At 4 months of age, inclusions similar to NFTs appeared in the cortex and significant neuronal loss $(\sim 60 \%)$ was detected in the hippocampus (Santacruz et al., 2005). Cognitive impairment became manifest at $2.5 \sim 4$ months of age by morris water maze (Ramsden et al., 2005). It is still under debate whether NFTs initiate neurotoxic events or it can be a protective response of segregating harmful oligomers from executing their function. By using tetracycline inducible expression system, Santacruz et al., 2005 provided a new insight on clear dissociation of neuronal loss and NFTs, suggesting that NFTs alone are not sufficient to cause neurodegeneration and cognitive deficits. Subsequently, Tau P301S (PS19) transgenic mouse was generated to overexpress 4R/1N isoform of P301S tau under the control of prion promoter. Slight synaptic loss in 3 month-old PS19 preceded the filamentary tau inclusions at 6 months of age at which spatial memory deficits were observed in morris water maze, followed by severe neuronal loss at 9 months of age (Yoshiyama et al., 2007).

Recently, rTgTauEC was generated to express human tau P301L mutation preferentially in the entorhinal cortex. Trans-synaptic tau propagation was revealed in the brain of rTgTauEC where tau pathology spreaded from entorhinal cortex to functionally connected neighboring brain regions. At 3 months of age, misfolded tau was detected by Alz50 staining in the entorhinal cortex of rTgTauEC (de Calignon et al., 2012). Gallyas silver-positive paired helical filaments, and both thioflavin $\mathrm{S}$ positive tangles and neuronal loss, were observed in the rtgTauEC at 18- and 24-months of age, respectively (de Calignon et al., 2012). There was no dramatic behavioral deficit in this mouse model. Polydoro et al, 2014 indicated that impairment of electrophysiological properties such as a decrease in LTP occurred even before tangle formation in rTgTauEC. More recently, $\mathrm{APP} / \mathrm{PS} 1 /$ htau transgenic mice were generatedto incorporate human AD genetics without overexpression of mutation in disease-linked proteins. This mouse model successfully recapitulated late-onset AD pathology by displaying age-dependent pathological changes such as $A \beta$ plaque formation and tau hyperphosphorylation, and behavioral changes including exaggerated fear response and motor deficits (Guo et al., 2013). 


\section{Protein Quality Control and Stress in Neurodegeneration}

Research on neurodegenerative diseases has focused on identifying the causes of developing misfolded protein aggregates in the brain and how it could be prevented or reduced for treating these devastating diseases. Despite clinical classification of AD and PD as distinct entities, the concept that they may share common genetic and pathogenic mechanism has been emerged and supported by numerous studies (Golde et al., 2013, Moussaud et al., 2014). It should be noted that protein aggregation, a common hallmark of neurodegenerative disease, could result from a failure of cellular defense system called protein quality control. Protein quality control is a collective term to describe cellular pathways that function in maintaining proteostasis. It is involved in ensuring proper protein folding, sensing damage of proteins, and promoting clearance of damaged protein.

Proteostasis is often challenged by various kinds of cellular stressors. In particular, proteotoxic stress refers to cytotoxic stimuli that increase the fraction of protein that are in an unfolded state, increasing the probability of forming aggregates. It includes stress conditions such as heat shock, oxidative stress, calcium dysregulation, and proteasome inhibition. In response to misfolded protein, stress response is activated to cope with proteotoxic stress.

Stress is not merely one of conseuqences of toxic events, but rather it might play a causative role in neurodegeneration. Even early life time experience of stress promoted $\beta$-amyloid plaque deposition in aged primates. (Merrill et al., 2011). There is an interesting model, namely "a stressor-threshold model of selective neuronal vulnerability", describes the relevance of stress to neuronal death. According to this model, gradually increasing stress differentially affects stress-resistant and stressvulnerable neurons, which may underlie the etiology and progression of neurodegenerative diseases (Saxena et al., 2011). Compared to stress-resistant neurons, stress-vulnerable neurons tend to form a vicious cycle of increasing intracellular stressor load, which results from low threshold to interrupt cellular homeostasis processes. Aggregation and propagation of toxic species may eventually affect other less vulnerable neurons to increase their stressor load.

Consequently, disrupted proteostasis leads to proteotoxicity originated from misfolded proteins, which causes impairment of cellular function. Proteotoxic proteins are usually disease-linked proteins that when mutated produce aggregates and result in neurodegeneration, such as prion, tau, $A \beta$, and synuclein. Proteotoxicity is especially detrimental to neurons in the brain with poor regenerative capability (Morimoto et al., 2008). The mechanism underlying the failure of proteostasis is not clearly understood. There is growing evidence that restoration of degradative machinery and enhancing the activities of chaperones alleviate proteotoxicity derived from dysregulated proteostasis. Herein, we will discuss various types of cellular protective system to cope with cellular stresses and their role in neurodegeneration. 


\section{Cellular Stress Response (Heat Shock Response)}

After Italian geneticist Ferruccio Ritossa found a puffing pattern of chromosome in response to heat stress in Drosophila in 1962 (Ritossa et al., 1962), in 1974, this puffing could be explained by the discovery of an induction of selective genes now called heat shock proteins (Schlesinger et al., 1990). Not only heat but also other chemical stress that results in denaturing proteins (proteotoxic stress) could increase the synthesis of heat shock proteins. Heat shock proteins are named according to their molecular weight. They are generally referred to as stress protein (stress-responsive gene). Evolutionarily, heat shock response is a highly conserved stress response. On the other side, in 1976, the term of chaperone was first coined for nucleoplasmin that assists in the correct assembly of nucleosomes by preventing the aggregation of histones with DNA. Later, the usage of the term cwas extended to a subset of proteins whose main function is to help correct folding of newly synthesized proteins and the assembled subunits (Ellis et al., 1987). Many heat shock proteins turned out to have chaperone activity that participates in correct (re)folding of proteins that are new or damaged from cellular stress (Schlesinger et al., 1990). Unlike other cell types, heat shock response is considered to be attenuated in neurons (Marcuccilli et al., 1999). Non-neuronal cells in the brain such as glial cells showed significant Hsp70 induction after heat stress, which was not observed in neurons (Pardue et al., 1992). Primary cultured hippocampal neurons failed to turn on the transcriptional activity of HSF1 under stress (Marcuccilli et al., 1996). Therefore, the existence of heat shock response in the rat hippocampus homogenates (Higashi et al., 1995) may be attributable to the ability of glial cells, not neurons, to activate HSF1 (Marcuccilli et al., 1996). Stress response involves not only gene transcriptional activation but also promoting the degradation of malformed proteins through ubiquitin proteasome system.

\section{Ubiquitin Proteasome System and Neurodegeneration}

Ubiquitin proteasome system (UPS) is one of the major proteinase complexes responsible for selective degradation of many short-lived small proteins in eukaryotes. UPS is a key controller of gene transcription because of its ability to degrade cyclins and transcription factors. Its neuroprotective feature is attributed to the function in degrading misfolded proteins as a result of stress. Substrate proteins targeted by UPS should be conjugated with ubiquitin ( $\mathrm{Ub}$ ) via three consecutive steps; E1, ubiquitin activating enzyme, adenylylates ubiquitin, followed by its transfer of ubiquitin to cystine residue of E2, ubiquitin conjugating enzyme. Lastly, ubiquitin can be conjugated from E2 to substrate protein by E3, ubiquitin ligase that recognizes its substrate and catalyzes the degradation process. Intracellular protein with K48-linked poly-ubiquitin chains is likely to be recruited to proteasome for its degradation. In contrast to polyubiquitination that acts as targeting signal toward proteasome, monoubiquitination has been considered as to spatially regulate protein activity by controlling protein localization and trafficking. Substrates containing K48-linked ubiquitin chains are usually degraded by the $26 \mathrm{~S}$ proteasome, whereas those containing K63-linked chains may undergo a number of different processes, including trafficking and/or lysosomal degradation. In particular, 
ubiquitin ligase NEDD4 (neural precursor cell expressed developmentally downregulated protein 4) was first reported as potential modifier of $\alpha$-syn pathology (Davies et al., 2014). A NEDD4 family of E3 ligase consists of 9 members (NEDD4, NEDD4-2 (NEDD4L), ITCH, SMURF1, SMURF2, WWP1, WWP2, NEDL1, and NEDL2) highly conserved in eukaryotes. It is characterized by three functional domains: $\mathrm{C} 2$ domain, 2-4 WW domains, and a HECT-type ligase domain. NEDD4 represents an ancestral member of the family. NEDD4 has several important substrate proteins crucial for normal CNS function such as PTEN, IGF- and AMPA receptor (Yang et al., 2010). Kwak et al., 2012 reported that endogeneous NEDD4 protein levels were significantly increased in human neurodegenerative diseases patients including PD.

There has been substantial evidence of UPS impairment in synucleinopathy and other neurodegenerative diseases (Keller et al., 2000, Chen et al., 2006). The first hint on the link between UPS and neurodegeneration is that protein aggregates like NFTs highly comprise Ub-conjugated proteins (Koo et al., 1999). Intracellular inclusion bodies found in AD contain a mutant variant of ubiquitin called UBB+1 (Braak et al., 1991). UBB+1 quite stable in neurons is not degraded by UPS. Lewy bodies, a hallmark of synucleinopathy, consist of highly ubiquitinated $\alpha$-syn. Brains of both human $\alpha$-syn transgenic mice and human PD patients display proteasomal dysfunction (Chen et al., 2006). Post-translational modification of UPS subunits may account for a significant decrease in UPS activity in the hippocampus of AD compared to control brain (Keller et al. 2000). Tg2576 amyloid transgenic AD mouse model exhibited decreased UPS activity (Oh et al., 2005). Recently, Myeku et al., 2016 provided a new evidence of tau-mediated $26 \mathrm{~S}$ proteasome dysfunction regulated by cAMP-protein kinase A. Impairment of UPS took place even before the formation of inclusion bodies, which suggests a possibility that intermediate forms of protein aggregates may be sufficient to disrupt UPS function (Bennett et al., 2005).

\section{Cellular Stress Response in ER and Neurodegeneration: Unfolded Protein Response (UPR) as a Result of ER Stress}

As the major function of endoplasmic reticulum (ER) is protein folding and synthesis, ER should be highlighted as a key intracellular place regulating the proteostasis inside cells. Its role in protein synthesis for seceretory, membrane and organelle-directed proteins are responsible for approximately $30 \%$ of total proteome. In response to the burden of unfolded proteins in the lumen of ER, ER can initiate its unique stress response called unfolded protein response (UPR). UPR is initially initated to restore ER homeostasis. In ER membrane, three ER sensor proteins (PERK, IRE1 $\alpha$, ATF6) are present that send intracellular activation signals in each of distinct signaling pathways to control transcription and translation during ER stress (Schröder and Kaufman, 2005). In the absence of ER stress, HSP70a5 (BiP/GRP78), a Hsp70 family protein in ER, inhibits UPR activation via its physical binding to PERK (Bertolotti et al., 2000). Whereas UPR is initially trigered as an adaptive response to the disturbances in ER homeostasis, inducing molecular chaperones such as Hsp70a5 (BiP/GRP78), sustained chronic UPR activation as a result of unresolved ER stress eventually promotes 
cell death by inducing pro-apoptotic proteins such as CHOP primarily through the PERKeIF2-ATF4 pathway.

The presence of ER stress has been continuously identified in the cellular/animal models and human studies for all the major neurodegenerative diseases (Matus et al., 2011). Inconsistencies in the existence and functional outcome of UPR have been reported among studies from distinct mouse models of neurodegeneration. This is partially owing to the different methods used to assess UPR activation and difference in mouse age and tau transgene. Phosphorylation of both eIF2 $\square$ and PERK was highly detected in the substantia nigra of human PD, not in control cases (Hoozemans et al., 2007). Furthermore, PERK activation not only co-localized with NFTs but also significantly correlated with hyperphosphorlyated tau in the human AD brains (Hoozemans et al., 2005, Jeroen et al., 2009). Human brain specimens of other tauopathies such as FTD showed UPR activation (Nijholt et al., 2012). While PERK was activated in rTg4510 tau transgenic mice (Abisambra et al., 2013), APP transgenic Tg2576 mice did not show any sign of UPR activation (Lee et al., 2010). Therefore, it suggests that UPR activation observed in human AD brain may occur as a result of abnormal tau aggregates, independent of amyloid beta deposits.

Several studies demonstrate that protein aggregates have potential to disrupt ERrelated basic cellular functions that induce UPR. Overexpressed $\alpha$-syn considerably blocked ER-Golgi trafficking, which was rescued by modifying the ER-Golgi trafficking component gene, Rab1 (Cooper et al., 2006). During ER stress, in parallel with UPR, ER induces endoplasmic-reticulum-associated protein degradation (ERAD), a cellular pathway for targeting misfolded protein to the proteasomal degradation. Abisambra et al., 2013 provided an evidence that overexpressed human tau P301L impaired ERAD and induced UPR. Mutant tau was physically associted with ERAD heterocomplex VCP and HRD1 in rTg4510 tau transgenic mice. As a result, ERAD substrate proteins failed to be properly degraded and therefore accumulated in $\mathrm{rTg} 4510$ and human AD brain.

Conversely, UPR activation seems to play a causautive role in tauopathy. Chemically induced ER stress led to tau hyperphosphorylation possibly mediated by GSK3 $\beta$ activation (Sakagami et al., 2013). Furthermore, UPR activation may contribute to tau accumulation by interrupting binding of tau to CHIP E3 ligase and inhibiting its proper degradation (Sakagami et al., 2013). Genetic deletion of PERK prevented deficits in both synaptic plasticity and spatial memory in APP-PS1 AD mouse model (Ma et al., 2013). Thus, it is likely that chronic UPR activation eventually contributes to synaptic dysfunctions manifested in AD. Hoglinger et al., 2011 showed the association of polymorphisms in the EIF2AK3 locus, a gene encoding PERK, with progressive supranuclear palsy, revealing a genetic linkage between UPR activation and tau pathology. Given its complexity of displaying both protective and apoptotic effects depending on the cellular context, it is necessary to understand which part of the UPR is desirable to target and at what particular stage of disease progression. 


\section{Autophagy-Lysosomal Pathway and Neurodegeneration}

Autophagy is an essential degradation process for proper turnover of long-lived proteins and entire organelles, such as mitochondria, inside the cell. Constitutively active autophagy is highly required for neuronal survival as its failure itself could initiate formation of ubiquitinated inclusion bodies and potentiate neuronal loss (Hara et al., 2006). Autophagy can be strongly induced by heat- and ER stress to promote a clearance of misfolded proteins, which is thus considered a neuroprotective mechanism (Ogata et al., 2006, Zhao et al., 2009). Stress-responsive transcription factor HSF1 is actively involved in autophagosome formation by binding to Atg7 promoter for transactivation (Desai et al., 2013). During ER stress, autophagosome formation was mediated by IRE1 JNK pathway (Ogata et al., 2006). Similar to UPR, autophagy is a protective response under mild stress but it triggers cell death in extreme conditions. It is important to recognize that the consequence of autophagy activation is largely determined by the specific mediator of autophagy active in the cellular context.

There are three subtypes of autophagy: macroautophagy, microautophagy, and chaperone-mediated autophagy, all of which are involved with autophagy-related genes (ATGs) and associated enzymes. Chaperone-mediated autophagy (CMA) is a very specific pathway where Hsc 70 complex binds to specific substrates via recognition site (KFERQ-like sequence) followed by binding of these substrate/chaperone complex to LAMP2A receptor in the lysosome for degradation. However, macrophagy is a nonspecific, bulk degradation process that involves the formation of autophagic vacuoles that finally fuse with lysosome, called autophagolysosomes. mTOR is a critical regulator of autophagy induction, and autophagy is known to be suppressed by mTOR activation that could be released by stress-related kinase AMPK signaling. Three downstream kinases such as ULK1, ULK2, and ULK3, could induce activation of Class III PI3K complex containing Beclin 1, the mammalian orthologue of yeast Atg6. ATG is the unified nomenclature for naming yeast genes involved in non-selective autophagy (Ohsumi et al., 2014). Currently, 37 ATG genes have been identified in yeast, and these genes seem to be highly conserved from yeast to human. A family of ATGs are involved in the specific steps in autophagy induction and cytoplasmic sequestration. Atg5 and LC3 II complexes regulate autophagosome formation. During autophagy, a cytosolic form of LC3 I is conjugated to phosphatidylethanolamine to form LC3 II which is further recruited to autophagosomal membranes.

Initial identification of autophagosome accumulation in the patient brains with all major neurodegenerative diseases suggests a possibility that autophagy impairment contributes to the pathogenesis of proteinopathy (Wu et al., 2011). The central role of autophagy in maintaining proteostasis has subsequently been demonstrated by studies from several mouse models and human brain. Mice lacking Atg5 gene developed cytoplasmic inclusion bodies and motor deficits (Hara et al., 2006). Genetic inhibition of Beclin1 exacerbated amyloid pathology by accelerating both intracellular and extracellular amyloid beta deposition in APP transgenic AD mouse model (Pickford et al., 2008). Consistently, reduced Beclin1/Atg6 levels were detected in the post-mortem human AD brain tissues (Pickford et al., 2008). Enhancement of autophagy by 
rapamycin, an inhibitor of mTOR, attenuated AD-like deficits in V717F APP mouse model (Spilman et al., 2010). To achieve a goal of clearing proteins encaptured by autophagosome, in addition to proper function of autophagosome formation, sufficient lysosomal biogenesis and function should be equally considered. Expansion of the lysosomal system found in early stages of AD brain might be a compensatory protective response (Barrachina et al., 2006, Khurana et al., 2010). Lysosomal structural protein LAMP1 in the limiting lysosomal membrane was upregulated in the human AD brains and AD mouse models (Barrachina et al., 2006). It is notable that there was negative correlation between LAMP1 expression and hyperphosphorylated tau accumulation in individual cortical neurons in human AD brain, suggesting a decrease in LAMP1 expression upon the formation of tangles resistant to degradation (Barrachina etal., 2006). Polymorphisms in a gene encoding Cathepsin D, a lysosomal aspartyl protease, have been associated with AD onset (Papassotiropoulos et al., 2000). Lysosomal dysfunction as a result of cathepsin inhibition was shown to be critical for tau cleavage and toxicity in Drosophila tauopathy model (Khurana et al., 2010).

\section{Heat Shock Factor 1 Regulation and Neurodegeneration}

HSF1 is involved not only in heat shock response but also in development, tumorigenesis, differentiation, and aging. HSF1-mediated transactivation of genes is not only limited to molecular chaperones but also includes synaptic gene expression (Chen et al., 2014, Wang et al., 2016). Thus, it is increasingly recognized that HSF1 protein is important for synaptic integrity and memory consolidation process (Hooper et al., 2016). Treatment of Hsp90 inhibitor, 17-AAG, led to increase synaptic proteins including PSD95 in primary neurons, dependent on HSF1 activation (Chen et al., 2014). In addition, boosting HSF1 activity by CNS-permeable Hsp90 inhibitior, OS47720, atttenuated cognitive deficits in the Tg2576 AD mouse model by upregulating expression of key synaptic genes such as BDNF (Wang et al., 2016). Synaptic depolarization could induce HSF1 activation in neurons, suggesting the relevance of HSF1 activation to synaptic activity (Hooper et al., 2016). Non-canonical mechanism of neuroprotective HSF1 function was recently discovered by Verma et al., 2013. This study indicated that HSF1 activation was neuroprotective even in the circumstance irrelevant of proteotoxic stress. They also demonstrated that the beneficial effect of HSF1 activation was not necessarily mediated by trimerization-associated upregulation of downstream genes. This novel trimerization-independent mechanism of HSF1 was proven to be effective in the conditions of huntingtin-induced toxicity (Verma et al., 2013).

It should be noted that there have been increasing reports on the linkage between intracellular level of HSF1 and neuronal survival/death (Jiang et al., 2013, Kondo et al., 2013, Liu et al., 2011, Verma et al., 2013), suggesting that HSF1 loss may be a major contributor to neurodegeneration. Jiang et al., 2013 identified an important role of HSF1 expression in the cerebellum of $A \beta$-infused AD rat model. Not only Hsp70 and Hsp40 expression but also steady-state level of HSF1 protein was significantly reduced in the cerebellum of this AD model compared to that of control rats. They demonstrated that enhancing HSF1 expression in the cerebellum of AD rat mitigated the loss of Purkinje 
cell bodies. Around the same time, Kondo et al., 2013 reported that HSF1 protein expression influenced pathogenic androgen receptor accumulation in the mouse model of spinal and bulbar muscular atrophy (SBMA). This mouse model overexpresses mutant androgen receptor gene harboring the expansion of a CAG repeat. Enhancing HSF1 expression in the brain lesion attenuated pathogenic protein accumulation and neuronal atrophy. On the other hand, HSF1 haploinsufficiency significantly promoted the expansion of disease-affected areas in the brain of SBMA mouse model.

\section{Structure and Function of HSF1}

The family of heat shock factors including HSF1, HSF2, HSF3, and HSF4 is evolutionarily highly conserved transcriptional factors responsible for the expression of heat shock proteins. Major HSFs found in mammals are HSF1, HSF2, and HSF4 while Drosophila, Caenorhabditis elegans, and yeast express only HSF1. In particular, HSF1 is a master regulator of heat shock response (chapter 1.2.1) that governs the transcriptional activation of chaperone genes during stress in eukaryotes and participates in a multitude of physiological processes. Upon stress, inactive HSF1 mainly located in the cytoplasm trimerizes and translocates into the nucleus where it binds to cis-acting heat shock elements (HSEs, extended repeats of the sequence nGAAn) in the promoters of stress responsive genes. The crucial function of HSF1 in regulating stress genes cannot be compromised (compensated?) by other HSF family members. HSF1 depletion in mice impaired thermotolerance by abolishing the induction of heat shock genes (McMillan et al., 1998).

HSF1 protein is 529 amino acids with 5 structural domains (DNA binding domain, two oligomerization domains, regulatory domain, trans-activating domain) (Figure 1-1). First, oligomerization domain encompassing leucine zipper-like domain (HR-A/B) located between DNA binding domain and regulatory domain and HR-C located between regulatory domain and trans-activating domain regulates the trimerization of HSF1 (Rabindran et al., 1993). Each unit (HR) is composed of an amphiphilic helix and hydrophobic heptad repeats. Hydrophobic amino acids repeats serve as a contact region for HSF1 each other. The interaction of HR-C with HR-A/B hampers this intermolecular hydrophobic structure. DNA binding domain located in the N-terminal $\sim 100$ amino acids is the most preserved region within the HSF family. Its looped helix-turn-helix feature enhances the stability of DNA-bound HSF1 trimer by protein-protein interactions without direct interaction with DNA. Each HSF1 monomer in trimerization can bind to HSEs in the major groove of the target gene promoters. The DNA-binding affinity of HSF1 tends to increase in trimeric HSF1 than monomeric HSF1. HSF1 target genes usually have more than one HSEs in their promoters, allowing simultaneous strong binding of HSF1 trimers. Trans-activation domain (TAD), the Cterminal 150 amino acids comprising two distinct domains TAD1 (401-420 a.a) and TAD2 (431-529 a.a), regulates transcriptional activation of target genes. It has been identified that acidic residues within TADs are involved in transcriptional initiation whereas hydrophobic residues mediate translational elongation step (Brown et al., 1998). 


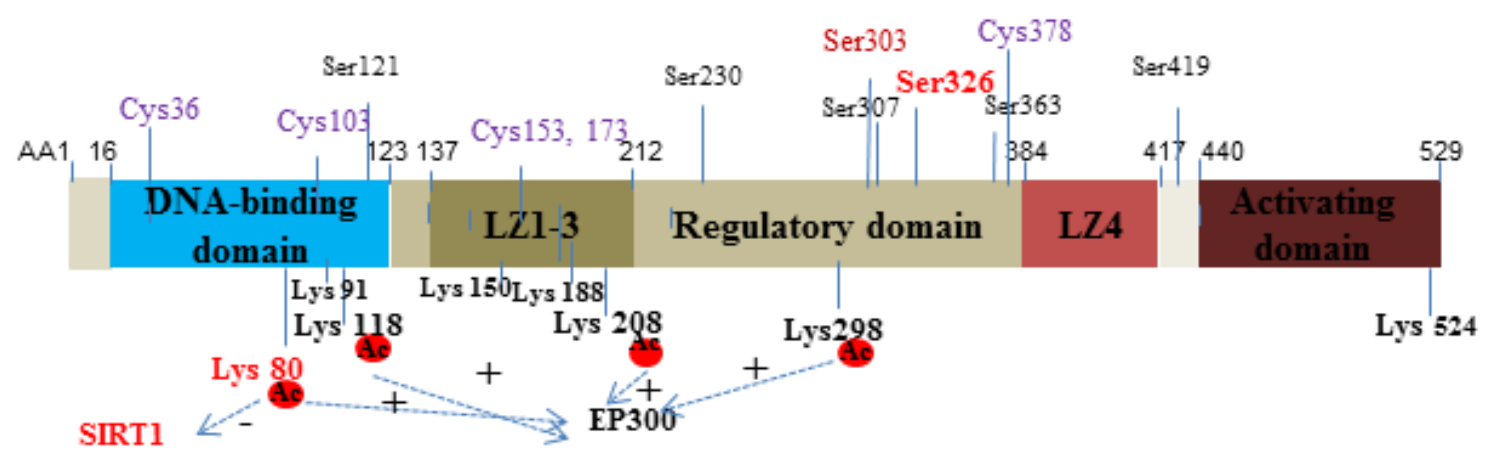

Figure 1-1. HSF1 Regulation by Post-Translational Modifications.

Oxidative posttranslational modification (Ox-PTM) of Cys residues (Cys36, Cys103, Cys 153, Cys173, Cys 378) can regulate HSF1 function. Phosphorylation of Ser230 and Ser326 is positively correlated with HSF1 activity. Ser121, Ser 303, and Ser307 are phosphorylation sites identified to suppress HSF1 activity. Lysine residues regulated by SIRT1 and EP300 are represented. 
Without stress, regulatory domain (212-384 a.a) located between HR-A/B and HR-C suppresses the HSF1 TAD (Green et al., 1995). The inhibitory effect of regulatory domain on HSF1 transcriptional activation can be modulated by post-translational modifications on multiple residues that reside in the regulatory domain.

\section{Regulation of HSF1 Activation}

HSF1 activation can be regulated by protein-protein interactions and posttranslational modifications. First, in the absence of stress, HSF1 activity is mainly inhibited by its binding to heat shock proteins including Hsp70 and Hsp90. Therefore, currently well-known HSF1 activators such as 17-AAG mostly act through inhibiting Hsp90. More importantly, post-translational modification of HSF1 enables fine-tuning HSF1 regulation in response to stress. Before this work, the consequence and mechanism of HSF1 protein modification were exclusively revealed by studies in non-neuronal cells.

Phosphorylation. Twelve serine residues of HSF1 have been identified by mass spectrometry (Ser(S)121, S230, S292, S303, S307, S314, S319, S326, S344, S363, S419, and S444). Phosphorylation of Ser303 and Ser307 in the regulatory domain has been clearly shown to suppress HSF1 activity (Kline et al., 1997, Neef et al., 2011), and these two sites are considered as conserved motifs for GSK3 and ERK1. GSK3 and other mitogen-activated protein kinases suppress HSF1 activity through phosphorylating Ser303 residue (Kline et al., 1997). Phosphorylation of HSF1 on Ser121 residue in the DNA binding domain by MAPK2 represses HSF1 activity by promoting its binding to Hsp90 protein (Wang et al., 2006). In contrast, Ser230 and Ser326 present in the regulatory domain are the phosphorylation sites positively associated with HSF1 activation. It is possible that CaMKII kinase activates HSF1 protein by regulating Ser230 (Holmberg et al., 2001).

Acetylation. In regards to regulation of HSF1 acetylation, K80 located in the DNA binding domain is the most characterized site whose deacetylation showed a strong positive correlation with HSF1 activity (Westerheide et al., 2009). Sirtuin 1 (SIRT1), (discussed in detail in chapter 1.3.5), is an enzyme found to be responsible for HSF1 activation by deacetylating HSF1 K80 (Westerheide et al., 2009). This work also demonstrated for the first time that $\mathrm{K} 80$ residue in HSF1 protein tends to be acetylated upon both heat- and proteotoxic- stress. It is notable that downregulation of SIRT1 that deacetylates HSF1 K80 site suppressed HSF1 binding to HSEs that exist in the promoters of downstream target genes (Westerheide et al., 2009). HSF1 acetylation state can be regulated not only by deacetylase SIRT1 but also by acetyltransferase EP300. In HeLa cells, EP300 targeted K118-, K208-, and K298- residues in HSF1 protein (Raychaudhur et al., 2013). In particular, acetylation of K118 by EP300 was found to occur in response to heat shock stress (Raychaudhur et al., 2013). Knocking-down EP300 in HeLa cells reduced the steady-state level of HSF1 protein, suggesting its new role on HSF1 protein stability (Raychaudhur et al., 2013). In conclusion, HSF1 acetylation has two major functions, regulating both HSF1 activation and HSF1 protein stability during stress. 
Ubiquitination. Studies to understand HSF1 ubiquitination are very limited until now. FBXW7 ubiquitin ligase was identified to ubiquitilate HSF1 protein in cancer cells, leading to its degradation (Kourtis et al., 2015). Loss of FBXW7 resulted in nuclear HSF1 accumulation (Kourtis et al., 2015). HSF1 protein interacted with FBXW7 ligase through Ser303/Ser307 (Kourtis et al., 2015). Chaperone-dependent E3 ubiquitin ligase CHIP plays a crucial role in the ubiquitination of unfolded protein (Murata et al., 2001). Despite its direct binding to HSF1 protein, it does not appear to be involved in HSF1 degradation through UPS. Rather, functional CHIP was required for HSF1 transcriptional activation (Dai et al., 2003). Taken together, multiple post-translational modifications of HSF1 have been studied using cancer cell lines in relation to its activity state including acetylation and phosphorylation. However, regulation of HSF1 protein turnover remains relatively unexplored, especially in neurons.

\section{Heat Shock Proteins and Neurodegeneration}

Chaperone proteins constitutively expressed inside cells, such as Hsp90, Hsp40, and $\mathrm{Hsc} 40$, exert housekeeping functions. They participate in protein translation, folding of newly synthesized protein and cellular translocation of protein (De Maio et al., 1999). Co-localization of chaperones and toxic inclusion bodies may indicate irreversible sequestration of substrate protein, and/or disability to correctly refold and degrade misfolded protein. Cytosolic chaperones such as Hsp70 are often detected in extracellular senile plaques, which might be associated with chaperone binding to lipid raft before $A \beta$ secretion (Broquet et al., 2003). Manipulating chaperone proteins has been implicated as a potential therapeutic strategy for treating neurodegenerative diseases.

In tau transgenic mouse models, several Hsp90 inhibitors significantly reduced tau aggregates (Dickey et al., 2006, 2007, Luo et al., 2007). By studies from Dr. Petrucelli group, Hsp90 inhibitors were found to selectively promote clearance of tau phosphorylated at proline-directed Ser/Thr sites such as S202/T205, preferentially through UPS system (Dickey et al., 2006). CHIP interaction with Hsp90 was required for phosphorylated tau degradation, although HSF1 appeared not to play a role in CHIPHsp90 complex-mediated tau degradation (Dickey et al., 2007). Tortosa et al., 2009 reported that direct binding of Hsp90 to tau protein resulted in a conformational change of tau and promoted GSK3-mediated tau phosphorylation and filament structure formation. Tau phosphorylation is regulated by CDK5 as well as GSK3. p35 is a neuronal specific co-activator of CDK5. Hsp90 formed a complex with p35 and P301L mutant tau in JNPL3 tau transgenic mouse (Luo et al., 2007). In addition, Hsp90 inhibition reduced CDK 5 activity by downregulating $\mathrm{p} 35$ and promoted clearance of mutant tau overexpressed (Luo et al., 2007).

Hsp90 inhibition induces Hsp70 expression. The Hsp70 family includes both constitutively expressed Hsc70, stress-inducible Hsp70 in the cytoplasm and nucleus, and endoplasmic reticulum localized Grp78. Inducible Hsp70 can be recruited into synapses following hyperthermic stress (Moon et al., 2001). Exogenous expression of inducible Hsp70 at synapses mimicked the effect of thermal preconditioning (Kelty et al., 2002). 
Overexpressing Hsp70 in $\alpha$-syn transgenic mice reduced triton insoluble $\alpha$-syn aggregates and facilitated $\alpha$-syn degradation (Klucken et al., 2004, Donmez et al., 2012). In a drosophila model of PD, although overexpressed Hsp70 protein did not alter $\alpha$-syn inclusion, it was revealed to significantly prevent dopaminergic neuronal loss derived from $\alpha$-syn toxicity (Auluck et al., 2002). Furthermore, Hsp70 is likely to impede both amyloid and tau pathology in AD. Virally mediated Hsp70 overexpression supressed intracellular A $\beta$ accumulation by binding to APP (Magrane et al., 2004). Hsp70 could suppress $A \beta$ oligomerization by recognizing exposed hydrophobic region in $A \beta$ oligomers to modify the conformation of oligomers (Lu et al., 2014). Enhanced Hsp70 protein expression by geldanamycin, a Hsp90 inhibitor, increased both tau solubility and binding affinity to microtubules, preventing tau aggregation (Dou et al., 2003). Silencing Hsp70 resulted in the opposite outcome in vitro cellular model (Dou et al., 2003). Later, it was further suppprted by in vivo study showing that both soluble and insoluble tau protein were reduced in mice overexpressing Hsp70 compared to control WT. Hsp70 and Hsp40 both prevented an intramolecular conformational change in a mutant huntingtin fragment (Schaffar et al., 2004).

Small heat shock proteins are highly conserved chaperone proteins. These proteins with low molecular mass of 12-43 kDa include Hsp27, $\alpha \mathrm{B}$-crystallin and Hsp20. The common characteristic of small heat shock proteins is that they possess a conserved $\alpha$-crystallin domain and form oligomers. Induction of their expression upon various cellular stressors such as heat shock and ischemia is dependent on HSF1 transactivation. Hsp27 has been identified to be upregulated in in vitro and in vivo models of synucleinopathy and human DLB cases (Outeiro et al., 2006). Increased Hsp27 levels were also observed in human AD brain as well (Björkdahl et al., 2008). Hsp27 had an ability to bind to $A \beta$ to suppress its aggregation (Wilhelmus et al., 2006). In addition, Hsp27 preferentially bound to hyperphosphorylated, pathological form of tau in human AD samples (Shimura et al., 2004). Tau protein in NFTs appears to be positively correlated with the levels of Hsp90, Hsp40, $\alpha$-crystallin, CHIP as well as Hsp27 in human AD brain (Björkdahl et al., 2008). Triton soluble $\alpha$-syn, not insoluble aggregates, also showed a close correlation with these HSPs in the brains of DLB patients (Klucken et al., 2006). In addition to its association with misfolded protein in neurodegeneration, several studies pointed out its anti-apoptotic activity to suppress mitochondrial cell death pathways by demonstrating that Hsp27 activated protective kinase Akt/PKB and supreessed pro-apoptotic JNK pathway (Rane et al., 2003, Voss et al., 2007). Genetically overexpressing Hsp27 in APP/PS1 of AD mouse model significantly attenuated various symptoms including not only $A \beta$ accumulation but also memory dysfunction and neuronal death (Tóth et al., 2013). There exists a controversy over the consequences of Hsp27 expression in different HD mouse models. Transgenic overexpression of Hsp27 did not attenuate protein aggregation and disease progression in R2/6 of HD mouse model (Zourlidou et al., 2007). In contrast, in HD rat models overexpressing mutated $\mathrm{Htt}$ fragments via lentiviral delivery, Hsp27 not only rescued striatal dysfunction but also increased solubility of aggregates, leading to decrease in the size of nuclear inclusions (Perrin et al., 2007). 


\section{HSF1 Activators}

Direct HSF1 activator has not been identified. As Hsp90 is a major repressor protein of HSF1 protein (Zou et al., 1998), many drugs that attempt to boost HSF1 activity are designed to inhibit Hsp90 function (Figure 1-2). Well-characterized Hsp90 inhibitors include geldanamycin and 17-AAG, both of which block the N-terminal ATP binding pocket of ATPase activity. Their efficacy in ameliorating protein deposits and neurotoxicity was demonstrated in both AD and PD mouse models (Chen et al., 2014, Shen et al., 2005), while clinical success is limited mainly due to systemic toxicity and BBB permeability. Celastrol, a Hsp90 inhibitor, acts through binding to C-terminal domain of Hsp90, leading to inhibition of chaperone activity as well as promoting HSF1 activity. Celastrol treatment was shown to ameliorate amyloid beta pathology in Tg PS1/APPsw of AD mouse model (Paris et al., 2010). However, its therapeutic potential needs to be carefully determined because of the cytotoxicity involved with thiol reactivity (Kalmar et al., 2009). In addition to Hsp90 inhibitors, by using a humanized yeast-based high-throughput screen to search HSF1 activators, HSF1A was identified to enhance HSF1 activity and chaperone expression, which led to decrease in protein aggregation and toxicity in polyQ-expressing neuronal precursor cells (Neef et al., 2010). TRiC, a central ATP-dependent chaperonin complex, is a negative regulator of HSF1 activation in yeast and mammalian cells (Neef et al., 2014). HSF1A, a small molecule activator of HSF1, is hypothesized to activate HSF1 by inhibiting the interaction of TRiC/CCT with HSF1 (Neef et al., 2014).

Arimoclomol, an analog of bimoclomol, has an ability to induce HSPs expression only under stress conditions (Kieran et al., 2004, Vigh et al., 1997). It circumvents any side effects associated with non-specific targets of heat shock response by not inducing stress response in the absence of stress (Vigh et al., 1997). In SOD1(G93A) of ALS mouse model, arimoclomol treatment resulted in improvement of motor function and motorneuron survival, significantly increasing lifespan (Kieran et al., 2004). Riluzole, a FDA-approved drug for amyotrophic lateral sclerosis (ALS), was recently discovered to stabilize HSF1 protein (Yang et al., 2008). Riluzole appears to attenuate HSF1 degradation via CMA sicne suppression of LAMP2A, a receptor for CMA substrates, abolished the effect of riluzole to increase HSF1 protein expression (Yang et al., 2008). By elevating HSF1 expression, riuzole treatment increased not only HSPs expression but also glutamate trasporter 1 expression (Liu et al., 2011). The underlying mechanism of its stabilizing effect on HSF1 protein remains to be further studied. Despite the benefical effects of various HSF1 activators as demonstrated by numerous studies, in order to develop HSF1-based therapy, it should be considered together that HSF1 has a powerful multifaceted role in carcinogenesis (Dai et al., 2007).

\section{Aging-related Changes in HSF1 and SIRT1 Activity}

Sirtuins are a highly conserved family of protein deacetylase that influences various cellular processes including stress resistance, apoptosis, inflammation, and energy efficiency. Among seven homologues, SIRT1, a human homologue of SIR2, has 


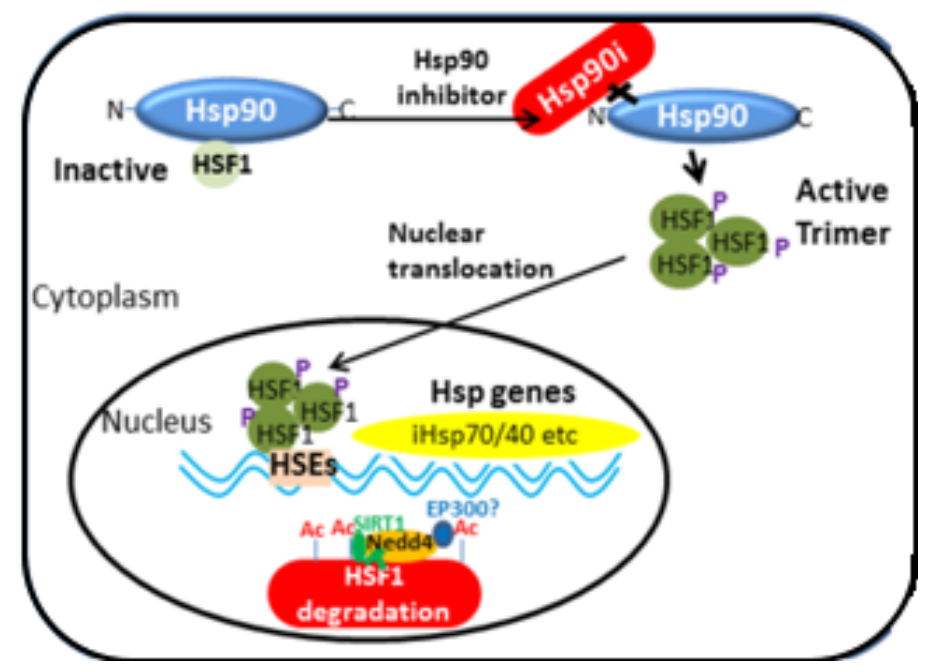

Figure 1-2. HSF1 Activation by an Hsp90 Inhibitor.

An Hsp90 inhibitor releases of HSF1 from Hsp90, leading to transactivation of Hsp genes. Post-translational modification of HSF1 protein such as acetylation and ubiquitination likely contributes to nuclear HSF1 degradation.

Data Source: Kim E, Wang B, Sastry N, Masliah E, Nelson PT, Cai H et al. (2016) NEDD4-mediated HSF1 degradation underlies $\alpha$-synucleinopathy. Hum Mol Genet 25:211-222 
been mostly characterized in mammals. SIRT1 has been highly implicated to promote longevity in a variety of organisms and emerged as a neuroprotective molecule in neurological diseases. There is a great body of evidence supporting the crucial roles of SIRT1 in both A $\beta$ and tau pathology. While SIRT1 overexpression ameliorated A $\beta$ production and plaque formation, SIRT1 deletion deteriorated amyloid plaque formation (Postina et al., 2004). Later, it was identified that acetylation of tau mediated by p300 acetyltransferase inhibited its degradation whereas SIRT1-mediated deacetylation of tau protein promoted ubiqutination and degradation of tau in neurons (Min et al., 2010). A linkage between SIRT1 decrease and disease progression was further supported by postmortem investigations. The levels of SIRT1 expression were inversely correlated with insoluble PHFs tau and A $\beta$ in human AD brain (Julien et al., 2009). Of note, SIRT1 activity is tightly linked to HSF1 activation as described above in Chapter 1.3.2.

Attenuation of stress response is a characterstic of senescence. Ben-Zvi et al., 2009 demonstrated in Caenorhabditis elegans that proteostasis network became compromised during aging. The steady-state level of HSF1 protein was downregulated in senescent WI38 fibroblasts when compared to young fibroblasts (Westerheide et al., 2009). Aging considerably reduced the levels of Hsp 70 mRNA induction following heat stress in various rat tissues including brain (Blake et al., 1991, Blake et al., 1990, Pardue et al., 1992). While 36 months- and 6 months-old rat brain were comparable in the levels of HSF1 protein expression, the aged brain clearly showed defects in the HSF1 ability to form trimerization (Akerfelt et al., 2010). Stress response is intimately linked to proper degradation of misfolded proteins via degradation pathways, and proteasome and autophagic activity decline with aging. Collectively, the critical role of HSF1 in proteinopathies ties in well with the aging-associated feature of neurodegenerat.

\section{Hypothesis, Rationale, and Specific Aims}

\section{Hypothesis}

We hypothesize that dysregulation of HSF1 protein represents an important mechanism underlying neurodegeneration (Figure 1-3).

\section{Rationale}

Neurodegenerative diseases are characterized by neuronal loss in the brain and the current classification of the diseases is largely based on disease-targeted region of the brain. The common characteristic of the affected areas in neurodegeneration is aggregation of misfolded proteins, for which they can be defined as proteinopathies in the brain. It has been widely accepted that a build-up of misfolded protein can be triggered and exacerbated by disrupted proteostasis. Proteostasis is mainly achieved by protein quality control including chaperones and degradation system. Much of the disease- 


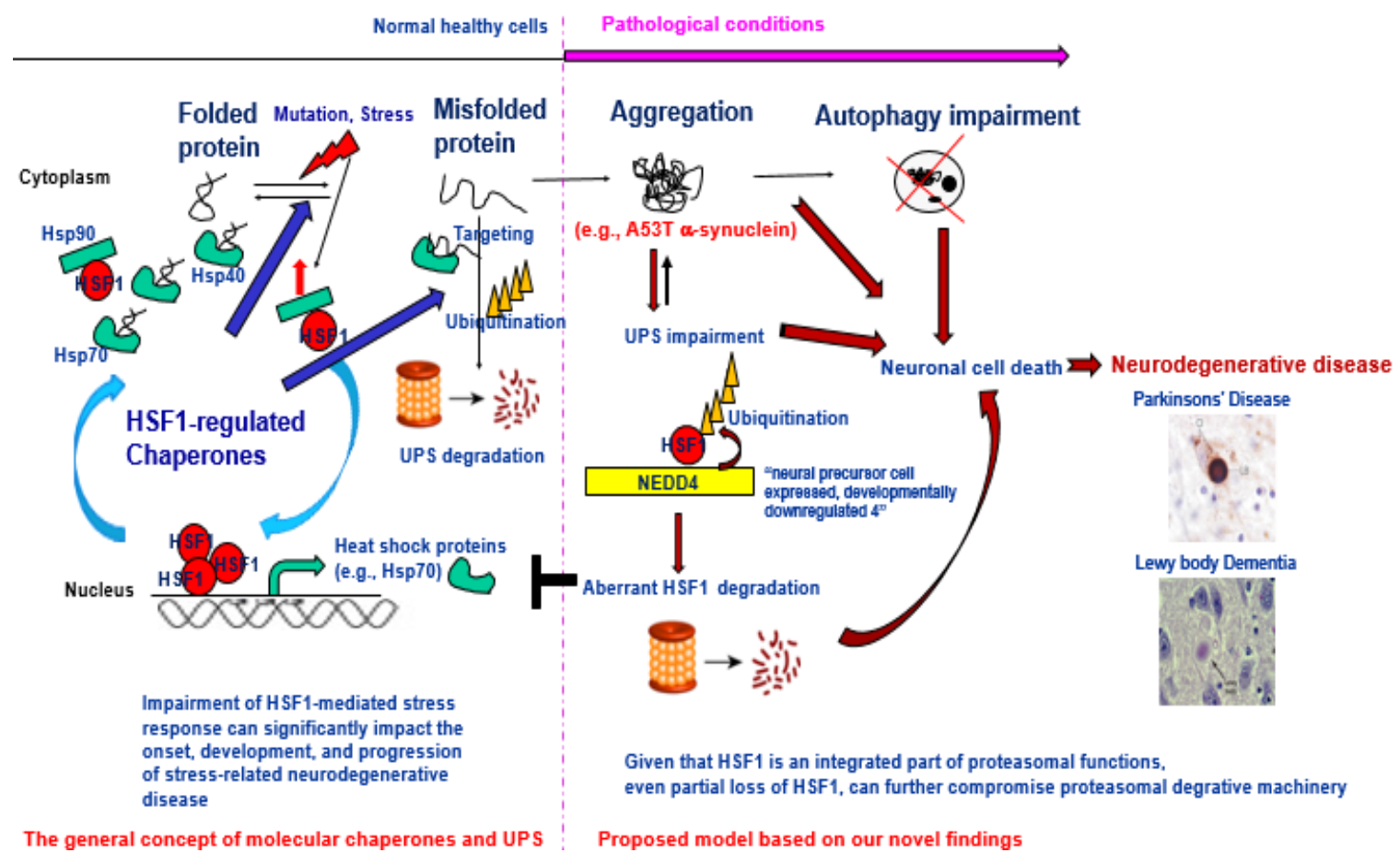

Figure 1-3. Aberrant HSF1 Degradation as a Common and Important Key Molecular Mechanism Underlying Neurodegeneration.

(Left) The general concept of molecular chaperones and UPS activated in normal healthy cells upon stress. (Right) Proposed model based on our novel findings.

Data Source: Kim E, Wang B, Sastry N, Masliah E, Nelson PT, Cai H et al. (2016) NEDD4-mediated HSF1 degradation underlies $\alpha$-synucleinopathy. Hum Mol Genet 25:211-222 
associated mutations implicated in neurodegeneration are directly or indirectly related with this defense system. Aging is a most critical factor accelerating the pathogenesis of neurodegenerative diseases, and the function of nearly all protein quality control systems appears to be compromised in aged neurons. In the lifetime, neurons can experience different kinds of stresses that include proteotoxic stress from misfolded protein. During stress response, chaperone proteins are upregulated to clear misfolded protein via UPS and/or autophagy. In contrast to healthy cells, aged cells and unhealthy cells under pathological conditions appear to lose their ability to fully activate a stress response, possibly leading to inclusion bodies formation in the brain.

Therefore, it is essential to understand the underlying mechanism that can explain the attenuated stress response in degenerating neurons and how failure of stress response contributes to the toxicity of protein aggregates. Most of all, HSF1, a master regulator of heat shock response, has been reported to be intrinsically related with other protein quality control systems (Dou et al., 2003, haggerty et al., 2011, Pierce et al., 2013). Since recent evidence strongly suggests important neuroprotective function of HSF1 in the brain, it is imperative to investigate how HSF1 protein is regulated in neurons undergoing chronic proteotoxic stress in AD and PD brain. This work may reveal the central role of HSF1 protein in neurodegeneration in cooperation with other cellular protective pathways.

\section{Specific Aims}

- Aim 1. To investigate whether and how HSF1 is dysregulated in synucleinopathy.

- Aim 2. To investigate whether and how UPR activation affects HSF1 dysregulation and vice versa in tauopathy.

- Aim 3. To investigate if HSF1 haplodeficiency affects hippocampal-dependent memory function. 


\title{
CHAPTER 2. METHODS
}

\author{
Animal and Human Studies
}

\section{Strains and Genotyping}

HSF1+/- mice (from Jackson laboratory, they came on the B6 background) were crossed once to C57BL/6J and maintained via intercrossing the F1 HSF1+/- mice. rTg4510 mice were produced by crossing Tau responder line with CaMKII $\alpha$-tTA transactivator line. PS19 mice were obtained on a C57BL/6J genetic background from Jackson laboratory. WT $\alpha$-syn transgenic mice were generated by Dr Cai's laboratory using the same strategy as described previously (Lin et al., 2009). Mouse tail was cut by a sterilized scissor. PCR was performed by using KAPA2G Fast PCR kit and GoTaq Flexi DNA Polymerase (Promega). HSF1 mice were genotyped by PCR with the following primer sequences: HSF1 mutant forward CGG TCG CTA CCA TTA CCA GT; HSF1 mutant reverse CCA AGG AGA TGT AGT GAG GTC TG; HSF1 wild type forward CTC CAT CTC CAG CCT ACA GC; HSF1 wild type reverse AGA GTG CTG CTG GAC AGA GG. PS19 mice were genotyped by PCR with the following primer sequences: Transgene reverse GGT ATT AGC CTA TGG GGG ACA C; Transgene forward GGC ATC TCA GCA ATG TCT CC; internal positive control forward CAA ATG TTG CTT GTC TGG TG; internal positive control reverse GTC AGT CGA GTG CAC AGT TT.

\section{Intraperitoneal Injection and Intra Substantial Nigra Viral Microinjection}

Mice were anesthetized by intraperitoneal injection of $10 \%$ chlor- al hydrate 3.5 $\mathrm{ml} / \mathrm{kg}$, and were stereotaxically fixed and injected with AAV2 or AAV5-CBA- $\alpha$-syn virus into the right substantia nigra of brains $\left(5.25 \times 10^{13} \mathrm{vg} / \mathrm{ml}, 1.5 \mu 1\right.$ at $0.5 \mu \mathrm{l} / \mathrm{min}$ speed, anter- ior fontanelle $4.4 \mathrm{~mm}$, sagittal suture (right) $1.3 \mathrm{~mm}, 8.5 \mathrm{~mm}$ under the skull). Viruses were generously provided by The Michael J. Fox Foundation for Parkinson's disease Research. Samples of different brain regions from single or double $\alpha$-syn transgenic mice were used for biochemical examination. To induce UPR activation, five month-old C57BL6 mice were intraperitoneally administered with thapsigargin $(2 \mathrm{mg} / \mathrm{kg})$ dissolved in PBS with 10\% DMSO.

\section{Histology}

Mouse cardiac perfusion was performed by a steady flow injection of phosphate buffered saline (PBS) and 4\% paraformaldehyde (PFA) for fixation. A double-staining protocol was used to compare Tau46 immunoreactivity to thioflavin S staining of plaques in floating sections from HSF+/- and WT. Monoclonal Tau46 antibody recognizes endogenous levels of total tau protein. The immunostaining procedure was performed 
before thioflavin S staining. We followed the procedure for thioflavin S staining described in Ly et al., 2011. For p-PERK (Thr980) and p-Tau (Ser202/Thr205) staining, images were captured under a confocal microscope (Olympus America, Center Valley, PA, USA). Mouse brain slices co-stained with Thioflavin S and Tau46 were observed by Olympus fluorescent microscope and a confocal microscope.

\section{Synaptosomal Membrane Preparation and Sarkosyl Insolubility Assay}

Mouse hippocampus was homogenated into sucrose-HEPES buffer ( $4 \mathrm{mM}$ HEPES pH 7.4, 3 mM Sucrose, 2 mM EGTA, containing protease and phosphatase inhibitors). Centrifuge the homogenate at $1,000 \mathrm{~g}$ for $10 \mathrm{~min}$ at $4{ }^{\circ} \mathrm{C}$ to remove the pelleted nuclear fraction. The resulting supernatant from further centrifugation at 15,000 $\mathrm{g}$ for $15 \mathrm{~min}$ at $4{ }^{\circ} \mathrm{C}$ yielded the crude synaptosomal pellet. Resuspend the resulting pellet into sucrose-HEPES buffer. The resulting supernatant from further centrifugation at $15,000 \mathrm{~g}$ for $15 \mathrm{~min}$ at $4{ }^{\circ} \mathrm{C}$ was discarded to remove contaminants. The pellet was then lysed in $4 \mathrm{mM} \mathrm{pH} 7.4 \mathrm{HEPES}$ and incubated at $4{ }^{\circ} \mathrm{C}$ for $30 \mathrm{~min}$. The resulting pellet from further centrifugation at $25,000 \mathrm{~g}$ for $30 \mathrm{~min}$ contained synaptosomal membrane fraction. Sarkosyl-insoluble tau protein from mouse hippocampus was biochemically isolated as described in de Calignon et al., 2012.

\section{Behavior Tests}

Behaviors of $\sim 13$ month-old WT (HSF1+/+), $\sim 13$ month-old HSF1+/-, and 25 month-old HSF1+/- were examined by a set of behavior tests as below.

Open field test. Mouse was placed in an open field chamber $(47 \times 37 \times 20 \mathrm{~cm}$, Accuscan Instruments) and allowed to freely explore for $10 \mathrm{~min}$. Experiments were performed between 10 AM to 4 PM. Sixteen infrared beams could automatically measure total locomoter activity of mouse by beam-breaks. EthoVision XT and video tracking software were used to track the mouse movement. The size of center region was about 25 $\%$ of the total field. Measurements in the test included distance travelled, center duration, center frequency, center latency, total activity, movement (and rest) time, and etc.

Cross maze. Mouse was placed from one entry of a cross-shaped maze made of opaque plastic walls (arm/wall sizes: $50 \mathrm{~L} \times 10 \mathrm{~W} \times 30 \mathrm{H}, \mathrm{cm}$ ), and allowed to freely explore four different arms during the 5 min test. The pattern of arm choices was automatically recorded by Maze software. The spontaneous alternation rate was calculated by dividing the number of alternations by the number of total arm entries. One alternation was counted when mouse made successive entries into each of the four arms. The number of total entries into arms was also analyzed.

Elevated plus maze. To assess anxiety-related behaviors, mouse was placed in the entry of open arm and allowed to freely explore for 5 min session. The elevated plus maze apparatus consists of two facing open arms, two facing closed arms and a center 
zone (arm/wall sizes: $50 \mathrm{~L} \times 10 \mathrm{~W} \times 30 \mathrm{H}$, cm; Runway height from floor: $55 \mathrm{~cm}$ ). This test was to measure the time spent in open or close arms. The total number of arm entries was also measured as a reference of general activity.

Fear conditioning test. Fear conditioning test was performed as previously described (Chen et al., 2014; Kittelberger et al., 2012). Mouse was placed in a steel chamber equipped with an overhead camera to monitor mouse movement $(30 \mathrm{~L}$ x $30 \mathrm{~W}$ x $25 \mathrm{H}, \mathrm{cm}$, Coulbourn Instruments LLC). Freezing percentage was determined by FreezeFrame software (Coulbourn Instruments LLC). Fear acquisition was performed on Day 1. The first three minutes was to record baseline activity and then mouse was subjected to a $2 \mathrm{~s}$ footshock $(0.7 \mathrm{~mA})$. After another one minute recording, mouse was given the second similar shock stimulus followed by additional one minute recording. Contextual fear retention was tested on the next day ( $24 \mathrm{hr}$ later). Three minutes recording was performed for the fear retention test; minute $1(\mathrm{ml}): 0-60 \mathrm{~s}$; minute 2 (m2): 60-120 s, minute 3 (m3): 120-180 s.

Morris water maze test. To examine spatial learning and memory, mouse was subjected to the Morris water maze test (Morris et al., 1984). Mouse was placed in a circular pool of water that was made opaque with non-toxic paint (114 cm in diameter), and allowed to freely swim. This test prompted mouse to escape from water onto a hidden platform $(10 \mathrm{~cm}$ in diameter $)$ under the surface of water $(0.5 \mathrm{~cm}$ in depth). There were four trials per day in the cued and hidden platform sessions. A visible sign for mouse to locate the platform was placed above the platform only during the cued sessions (day 1-4). For each trial in the hidden sessions, mouse started from different quadrants. Measurements of the test included the latency for mouse to locate the platform and total distance travelled. For a probe trial at the end of hidden sessions, mouse was allowed to swim for 1 minute in the pool where the platform was removed.

\section{Human Specimens}

Tissue specimens of Non-AD and AD patients were obtained from the Human Brain and Spinal Fluid Resource Center, which is sponsored by NIHDS/NIMH, National Multiple Sclerosis Society, Department of Veterans Affairs. Cases derived from shortpostmortem interval (PMI) autopsies from the University of Kentucky AD Center (UKADC) cohort (Schmitt et al., 2012). Premortem clinical evaluations and pathological assessments were as described previously (Nelson et al., 2010). Frontal cortical sections correspond to Brodmann Area 9. Tissue used for the biochemical analyses were snapfrozen during the autopsy in liquid nitrogen and then stored at $-80^{\circ} \mathrm{C}$. The inclusion/ exclusion criteria that were applied: PMI $<4 \mathrm{hrs}$; no evidence of frontotemporal dementia (clinically) or frontotemporal lobar degeneration (pathologically); no cancer in the brain parenchyma; and no large infarctions in the brain, or microinfarcts found within $3 \mathrm{~cm}$ of the brain tissue samples. The neuropathological features were assessed using standard neuropathological procedures as described in detail elsewhere (Nelson et al., 2009, 2010). Human snap-frozen inferior parietal (Brodmann Area 39) samples used for the study were provided by the Biobank at Uni- versity of Kentucky Alzheimer's Disease Center 
(Schmitt, et al., 2012). Neuropathologic diagnoses were as described previously and inferior parietal lobe tissue was used because when that is affected one can be confident of the 'diffuse/neocortical' subtype of DLB (Nelson et al., 2009-2). Whole brain lysates or tissue lysates from distinct brain areas of mice were used for western blotting.

\section{Biochemical Studies}

\section{Cell Cultures and Generation of Stable Cell Lines}

SH-SY5Y cells were cultured in Dulbecco's modified Eagles' me- dium (DMEM) supplemented with nutrient mixture F-12 and 10\% fetal bovine serum (FBS). Human embryonic kidney (HEK) 293 cells were grown in DMEM plus 10\% FBS. Cells were trans- fected with plasmids using Lipofectamine 2000 (Invitrogen) and harvested 2 days after transfection. N2a cells were cultured in a 1:1 mixture of DMEM and Opti-MEM containing $10 \%$ fetal bovine serum (FBS). Cells were transfected with plasmids using Lipofectamine 2000 (Invitrogen). For generation of stably transfected cell lines (N2a$\mathrm{Tau}_{\mathrm{RD}} \Delta \mathrm{K} 280$ ), G418 at $1 \mathrm{mg} / \mathrm{ml}$ was included in the culture medium. Primary cortical and hippocampal neurons were prepared from E17 rat embryos and maintained in neurobasal medium supplemented with $0.8 \mathrm{~mm}$ l-glutamine and B27. Primary neurons cultured for 10-18 days in vitro (DIV) were used for the study.

\section{Reagents and Plasmids}

Plasmid constructs used in transient transfection include full length tau (WT, R406W) and repeated domain tau constructs (Tau RD WT, Tau RD P301L, Tau RD $\triangle$ K280); pcDNA-SIRT1 WT; pcDNA-HSF1 WT, S303A and HSF1 $\Delta$ 156-226; pcDNA-HSP70a5 (BiP/GRP78). HSF1 WT and HSF1 S303A were generously given by Dr. Dennis Thiele at Duke University. HSF1 $\Delta 156-226$ (trimerization mutant) was cloned by using a method of site-directed mutagenesis (Agilent Technologies). Tunicamycin, thapsigargin, salubrinal, resveratrol, piceid, celastrol, rapamycin, and riluzole were all purchased from Sigma. siRNA oligomers for CHOP and BiP were purchased from Sigma and SIRT1 siRNA from Cell Signaling. RSV, MG132, cycloheximide, NAM, sirtinol were all from Sigma-Aldrich. Chloroquine diphosphate sulfate was from MP Biomedicals. Plasmid constructs used in transient transfection include EGFP-C1-WT $\alpha$-syn, pEGFP-C1- A53T mutant $\alpha$-syn and empty vector pEGFP-C1; pcDNA-SIRT1 WT and mutant SIRT1 H355A; pRC-CMV-NEDD4 WT and NEDD4 C866S; HSF1 WT; HA-Ub. HSF1 LPKY deletion mutant, HSF1 K80Q and K80R were cloned by using a method of site-directed mutagenesis (Agilent Technologies) 


\section{Protein Analysis by Western Blots}

Tissues and cells were lysed using RIPA buffer (10 mM Tris-Cl ( $\mathrm{pH} 8.0), 1 \mathrm{mM}$ EDTA, 1\% Triton X-100, 0.1\% sodium deoxycholate, 0.1\% SDS, $140 \mathrm{mM} \mathrm{NaCl}$ ) containing protease inhibitor (leupeptin, pepstatin A, phenylmethylsulfonyl fluoride (PMSF), aproptinin). The resulting lysates were subjected to western blots. Both monoclonal antibody (Santa Cruz, E-4) and polyclonal antibody (Cell signaling, 4356) were used to study HSF1 protein expression. Both antibodies detected $\sim 82 \mathrm{kDa}$ HSF1 but showed different banding patterns. SIRT1 (Millipore) and p-PERK/p-eIF2 $\alpha /$ eIF $2 \alpha / \mathrm{BiP} / \mathrm{CHOP} / \mathrm{Tau}$ (Tau46) (these six from Cell signaling) were also used in the study. Tau (Tau46) Mouse monoclonal antibody detects all six isoforms of tau based on the amino acid sequence. For phosphorylated tau, two specific antibodies of AT8 (MN1020) and AT100 (MN1060) were purchased from Thermofisherscientific. Western quantification was based on the intensity of interested signal using densitometry and ImageJ software program.

\section{In Vivo Ubiquitination Assay and Cycloheximide Chase Assay}

Transfected cells were lysed in buffer (2\% SDS, $150 \mathrm{mM} \mathrm{NaCl}, 10 \mathrm{mM}$ Tris$\mathrm{HCl}, \mathrm{pH} 8.0$ ) with $2 \mathrm{mM}$ sodium orthovanadate, $50 \mathrm{mM}$ sodium fluoride and protease inhibitors. The resulting lysates were boiled for $10 \mathrm{~min}$ and sheared by sonication. For immunoprecipitation, the HSF1 containing protein complexes were pull-down by antiHSF1 antibody, washed and eluted from beads via boiling and subjected to immunoblot using anti- ubiquitin antibody (Enzo Life Sciences). Western quantification was based on the intensity of interested signal using densitom- etry and ImageJ software program. In SH-SY5Y cells, $100 \mu \mathrm{M}$ (Sigma) cycloheximide was added for the indicated times in the presence or absence of MG132 24 48 h after transfection of empty or A53T vector with or with- out SIRT1 WT constructs. Cell lysates were then prepared for fur- ther analysis.

\section{RNA Analysis (PCR and qRT-PCR)}

Total RNA in HEK293 and SH-SY5Y cells was prepared by following the Trizol RNA Isolation Protocol (Life Technology). By using the High-Capacity cDNA Reverse Transcription Kit (Applied Biosystems), single-stranded cDNA was synthesized from total RNA. Mixtures of cDNA, SYBR Green MasterMix (5 Prime, Inc.), and primers were analyzed by Real-time PCR system (Invitrogen) to measure RNA levels.

\section{MTT (3-(4,5-dimethylthiazol-2-yl)-2,5-diphenyltetrazolium bromide) Assay}

Cells in a 24 well-plate were incubated with $10 \mu 1$ of the $12 \mathrm{mM}$ MTT stock solution in a total $100 \mu \mathrm{l}$ fresh culture medium for $2 \sim 3 \mathrm{hrs}$ at $37^{\circ} \mathrm{C}$. Reaction was stopped by incubation with $100 \mu \mathrm{l}$ of the SDS-HCl solution for $2.5 \mathrm{hrs}$. Absorbance at $570 \mathrm{~nm}$ was detected by Beckman Coulter DTX 880 multimode detector 


\section{Immunofluorescence Analysis}

SH-SY5Y cells and mouse brain tissues were fixed in 4\% paraformaldehyde, human brain was fixed in 10\% formalin, all followed by sectioning and then blocking and incubation of primary antibody overnight and then AlexaFluor-conjugated secondary anti- body. Fluores- cence image were captured by confocal and fluorescence microscopy (Olympus).

\section{Statistical Analysis}

All statistical analysis was performed by Student's t-test for two groups' comparison and one-way ANOVA with a Tukey test for multiple comparisons.

Quantitative data in all graphs were presented by means \pm SEM (standard error of mean). 


\section{CHAPTER 3. THE ROLE OF HSF1 PROTEIN REGULATION IN SYNUCLEINOPATHY*}

\section{Introduction}

Synucleinopathies are a major class of neurodegenerative dis-eases, including Parkinson's disease (PD) and diffuse lewy body (DLB). These pathologic conditions are defined by the presence of pathological $\alpha$-synuclein aggregates known as Lewy bodies. Disruption of protein quality control, interconnected cellular strategies of the ubiquitin proteasome system (UPS) and molecular chaperones, has been postulated to be involved in the patho-genesis of neurodegeneration underlying proteinopathies (Chen et al., 2011). Molecular chaperones composed of a set of heat shock proteins (HSPs) are considered a first line of defense against misfolded and aberrantly accumulated proteins like $\alpha$ synuclein aggregates that trigger the pathologic cascade and eventually lead to deficits in the overall protein clearance. For unknown mechanism, several cell types including neurons appear to be poorly adapted for chronic proteotoxic stress (Morimoto et al., 2008). Since the induction of HSPs is primarily determined by the activation of heat shock transcription factor 1 (HSF1), HSF1 has been regarded as an attractive target for treating neurodegenerative diseases (Neef et al., 2011). Multiple post-translational modifications of HSF1 have been studied in relation to its activity state, including acetylation, sumoylation and phosphorylation (Lindquist et al., 1986, Cotto et al., 1998, Zou et al., 1998, Shi et al., 1998, Morimoto et al., 1998). However, regulation of HSF1 turnover remains relatively unexplored, especially in neurons. It should be noted that there have been increasing reports on linking the intracellular level of HSF1 with neuronal survival/death (Jiang et al., 2013, Kondo et al., 2013, Liu et al., 2011, Verma et al., 2014), suggesting that HSF1 loss can be a major contributor to neurodegeneration. In this work, we discovered the 'neural precursor cell ex- pressed, developmentally downregulated 4 (NEDD4-1, NEDD4 herein)' (Yang et al., 2010, Donovan et al., 2013) likely being the E3 ligase of HSF1 in neurons under proteotoxic stress conditions. We speculate that aberrant HSF1 degradation is a common and important key molecular mechanism underlying neurodegeneration. Moreover, HSF1 deacetylation can attenuate this process via stabilizing HSF1 protein.

\footnotetext{
${ }^{*}$ Reprinted with permission. Kim E, Wang B, Sastry N, Masliah E, Nelson PT, Cai H et al. (2016) NEDD4-mediated HSF1 degradation underlies $\alpha$-synucleinopathy. Hum Mol Genet 25:211-222
} 


\section{Results}

\section{Overexpressed $\alpha$-syn Protein Promotes Ubiquitination and Degradation of HSF1 Protein via UPS}

To determine whether $\alpha$-synuclein ( $\alpha$-syn, herein after) aggregation altered HSF1 expression levels, we transiently transfected SH-SY5Y neuroblastoma and HEK293 cells with GFP-tagged wild-type (WT) $\alpha$-syn (GFP- $\alpha$-syn WT) or GFP-tagged A53T mutant $\alpha$ syn (GFP- $\alpha$-syn A53T). In both cell lines, A53T $\alpha$-syn overexpression $48 \mathrm{~h}$ post transfection caused more dramatic re- duction ( $>70 \%$ loss) in HSF1 protein expression than WT $\alpha$-syn (Figure 3-1A, C), without decreasing the mRNA levels of hsfl gene (Figure 3-1B). A53T mutant form of $\alpha$-syn is known to aggregate more rapidly than WT form. We could detect both Triton X-soluble and detergent-insoluble $\alpha$-syn aggregates in $\alpha$-syn transfected SH-SY5Y cells (Figure 3-1D). Insoluble WT $\alpha$-syn expression was increased by transfection of double concentration of WT $\alpha$-syn (WT $2 \mu \mathrm{g}$ ) (Figure 3-1D). This higher WT $\alpha$-syn aggregation resulted in $~ 50 \%$ loss of HSF1 protein (Figure 3-1A, C). In contrast to A53T $\alpha$-syn which resulted in HSF1 loss in both nuclear and cytoplasmic compartments, WT $\alpha$-syn only caused reduced HSF1 in the nucleus (Figure 3-1C).

We next assessed HSF1 protein stability by treating transfected cells with cyclohexamide (CHX). While HSF1 protein was stable in SH-SY5Y cells transfected with empty vector, it was greatly reduced in cells overexpressing A53T over time (Figure 3-1E), which was largely rescued by proteasome inhibitor MG132 but not by chloroquine (CQ, a lysosomal inhibitor). Of note, we noticed upshifted HSF1 bands in both control and A53T- trasnfected cells when blocking proteasomal degradation with MG132 (Figure 3-1E; denoted by *). This slower mobility-shifted band may be due to hyperphosphorylated HSF1 which correlated with hyperactivated HSF1 following heat shock and proteasomal stress (Holmberg et al., 2001).

\section{a-Syn-induced HSF1 Degradation is Mediated by the E3 ligase NEDD4}

UPS degradation is mediated by ubiquitin E3 ligase conjugating ubiquitin molecules to a lysine residue of substrate protein. Previously, we discovered that E3 ubiquitin-protein ligase NEDD4 expression was upregulated in the brain samples of PD patients (Kwak et al., 2012). The LPKY-motif in the endoplasmic reticulum localized transcription factor Spt23p was previously shown to interact with yeast Rsp5 (mammalian homolog NEDD4) (Schcherbik et al., 2004). We found the same motif in the DNA-binding domain of HSF1 (57-60 amino acid sequence). Therefore, we reasoned that NEDD4 may be involved in HSF1 protein degradation. Indeed, we observed significant enhancement of NEDD4 expression and in particular nuclear NEDD4 during $\alpha$-syn-induced HSF1 degradation (Figure 3-2A, B, and C). NEDD4 appeared to undergo active shuttling between nuclear and cytoplasmic compartments upon overexpressing A53T as compared with its perinuclear distribution in control cells, as detected by both subcellular fractionation assay and confocal microscopy (Figure 3-2B, C). 
Figure 3-1. $\quad \alpha-S y n$ Aggregation Causes HSF1 Degradation via UPS.

(A) Immunoblot assay showing the expression levels of HSF1. SH-SY5Y cells were transiently transfected with different concentrations of WT $\alpha$-syn or A53T $\alpha$-syn

plasmids. Quantification of the western blot images was done by densitometry (right, $\mathrm{n}=$ 3 , ***P $<0.001$, means $\pm \mathrm{SD}$ ). (B) Quantitative measurement of HSF1 mRNA levels normalized by GAPDH transcripts in transfected SH-SY5Y cells by qRT-PCR $(\mathrm{n}=3$, $* * * \mathrm{P}<0.001 ; * \mathrm{P}<0.05$, data represent means $\pm \mathrm{SD}$ ). (C) Subcellular fractionation assay to separate nuclear and cytoplasmic HSF1. Cells were transfected with different amounts of $\alpha$-syn (WT or A53T) constructs. (D) Following Triton X-100 fractionation, both insoluble and soluble $\alpha$-syn were detected by using $\alpha$-syn antibody instead of GFP antibody in transfected SH-SY5Y cells (arrow represents monomeric $\alpha$-syn of $18 \mathrm{kDa}$ size. *tetramer of $\alpha$-syn). (E) Proteosomal HSF1 degradation induced by A53T $\alpha$-syn. SH-SY5Y cells were treated with $50 \mu \mathrm{M} \mathrm{CHX}$ for various time points with or without pretreatment of $25 \mu \mathrm{M}$ MG132, $48 \mathrm{~h}$ after transfection. MG132 but not CQ $(50 \mu \mathrm{M})$ blocked HSF1 degradation (right panel). Control was transfected with empty vector (AE). (F) HSF1 ubiquitination in A53T $\alpha$-syn transfected cells. Co-immunoprecipitation of ubiquitin (Ub) with HSF1 using antibody against HSF1 was performed with protein extracts of A53T-transfected SH-SY5Y cells with or without MG132 treatment (left panel). GFP- $\alpha$-syn A53T was transiently co-transfected with various amounts of HAtagged ubiquitin (HA-Ub) into SH-SY5Y cells (right panel). The HSF1-complexes immunoprecipitated from lysates with anti-HSF1 antibody were then subjected to immunoblot using an antibody against ubiquitin. HSF1 abundance showed an inverse relationship to the level of ubiquitination. 
A
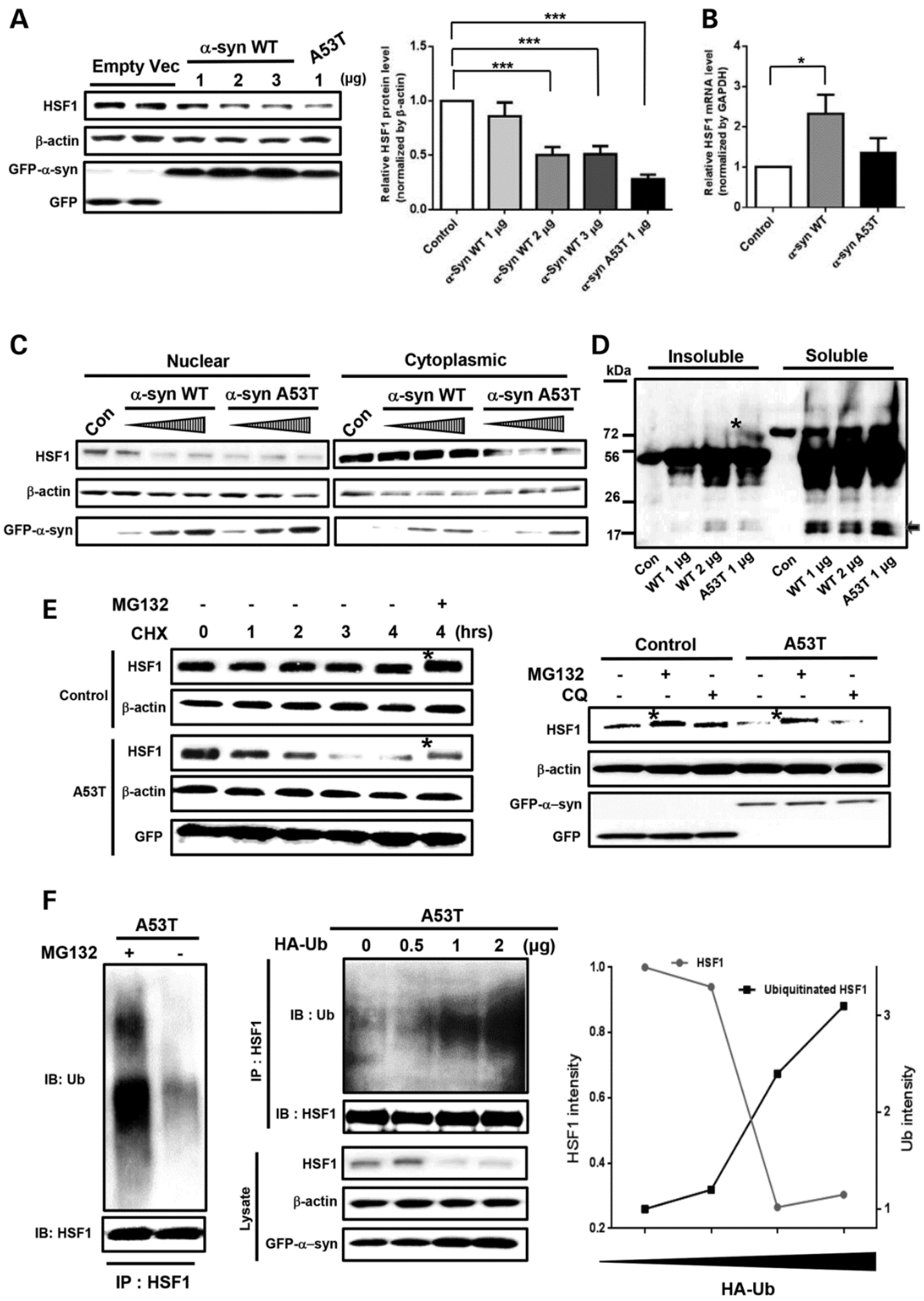
Figure 3-2. NEDD4 Promotes Ubiquitination and Proteasomal Degradation of HSF1 in Neuroblastoma cells Overexpressing A53T $\alpha$-syn.

(A) Elevated NEDD4 and decreased HSF1 protein levels by A53T $\alpha$-syn overexpression in SH-SY5Y and HEK293 cells. Cells were transfected with empty GFP vector (control), $\alpha$-syn WT or $\alpha$-syn A53T. (B) Intracellular NEDD4 protein expression (red) in SH-SY5Y cells with or without A53T $\alpha$-syn was visualized by immunocytochemistry. DAPI (blue) was used for nuclear staining. The merged images are presented in the bottom. Scale bar: $10 \mu \mathrm{m}$. (C) Subcellular fractionation assay to separate nuclear and cytoplasmic NEDD4. SH-SY5Y cells were transfected with $\alpha$-syn (WT or A53T). Lamin B1, a nuclear envelope marker, was shown to indicate nuclear fraction. (D and E) NEDD4 WT overexpression in SH-SY5Y cells transfected with A53T $\alpha$-syn further decreases HSF1, inducible Hsp70 and $\alpha$-syn levels and increases expression of pro-apoptotic factor Bax. SH-SY5Y cells were transfected with A53T $\alpha$-syn, along with NEDD4 WT, NEDD4 C866S or NEDD4 siRNA. Quantification of HSF1 protein expression was normalized to $\beta$-actin $(\mathrm{n}=3, * * \mathrm{P}<0.01$, means \pm SD). (F) NEDD4 WT overexpression facilitates ubiquitination of HSF1 in A53T-transfected cells (left panel). The interaction between NEDD4 and HSF1 (right panel). Three days after transfection of GFP-A53T or empty GFP vector alone or together with HA-Ub in SH-SY5Y cells, endogenously expressed HSF1 proteins were pull-down in whole-cell lysates, followed by immunoblot using anti$\mathrm{Ub}$ and anti-NEDD4 antibody (right panel). 
A

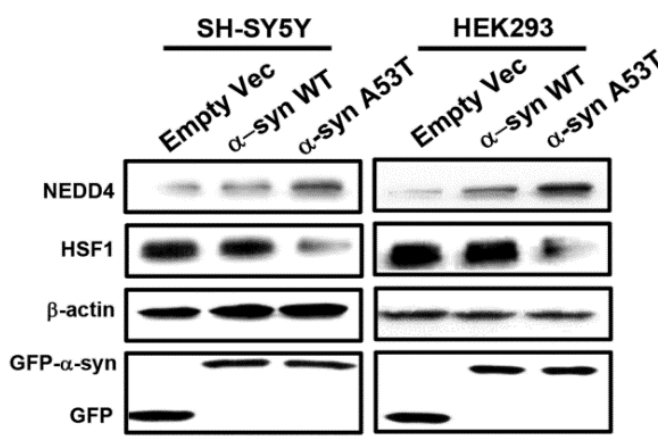

C

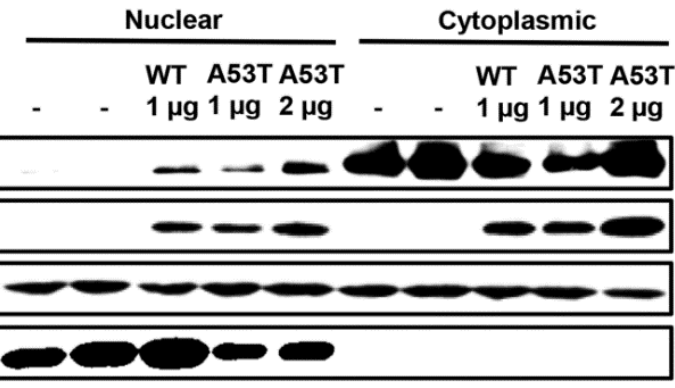

D

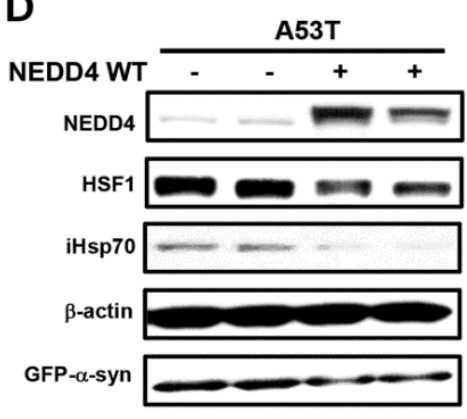

F
B
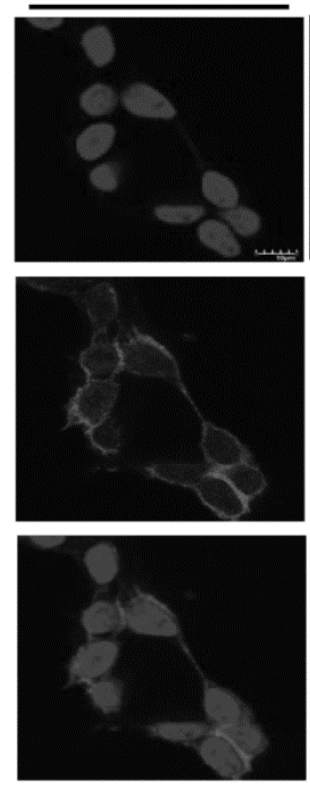

DAPI / NEDD4 (red)

E

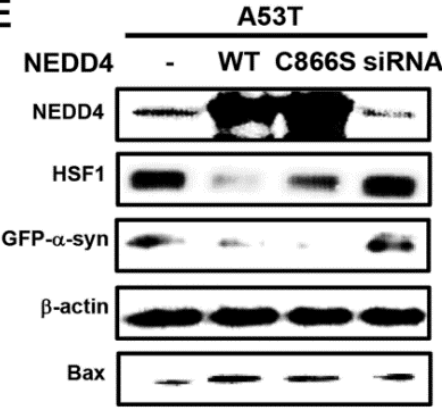

Empty vec A53T
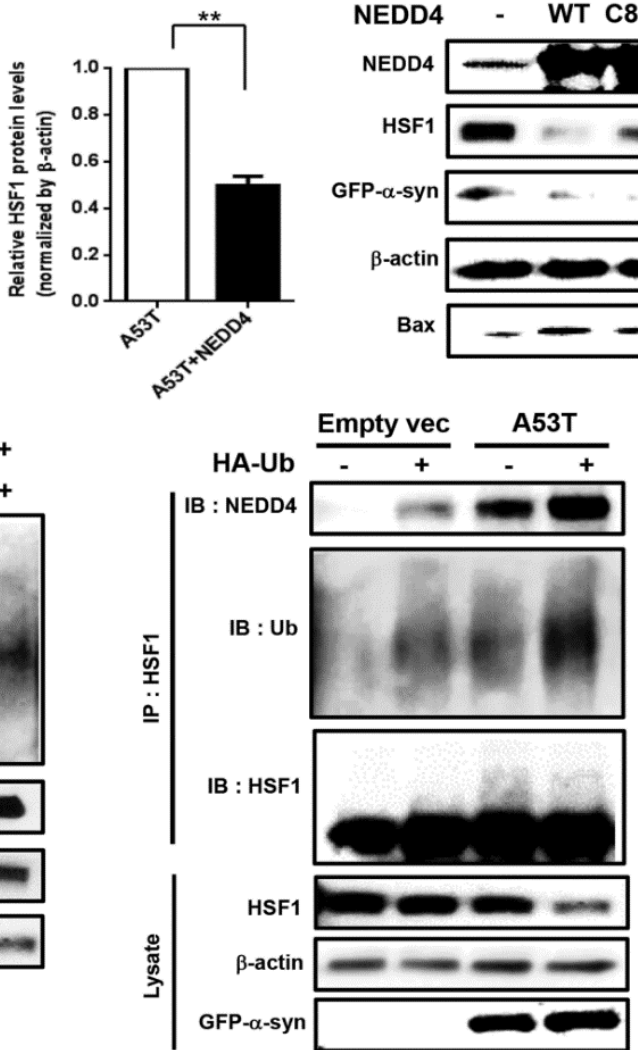
To investigate NEDD4-mediated HSF1 degradation, we overexpressed WT NEDD4 (NEDD4 WT) in A53T-transfected SH-SY5Ycells. It should be noted that A53T-transfected SH-SY5Ycells overexpressing NEDD4 WT showed $\sim 50 \%$ reduction of HSF1 along with decreased inducible Hsp70 (iHsp70) whose ex- pression is regulated by HSF1 activation (Figure 3-2D). On the contrary, overexpression of catalytically defective NEDD4 mutant (NEDD4 C866S) as well as NEDD4 siRNA failed to reduce HSF1 protein (Figure 3-2E), indicating that the ubiquitin ligase activity of NEDD4 was required for the loss of HSF1 protein. We then detected enhanced mono-and polyubiquitination of HSF1 in SH- SY5Y cells overexpressing NEDD4 WT (Figure 3-2F) which was further facilitated by expression of exogenous HA-Ub in both control and A53T-transfected cells (Figure 3-2F). Moreover, the strength of NEDD4- HSF1 interaction appeared to be positively correlated with the degree of HSF1 polyubiquitination and its degradation (Figure 3-2F). Taken together, we could conclude that despite the reduction in $\alpha$-syn (Figure 3-2D, E), degradation of HSF1 was further promoted by NEDD4 overexpression in A53T-transfected SH- SY5Y cells.

\section{Loss of HSF1 Protein Induced by $\alpha$-synucleinopathy in vivo}

We determined the protein expression profiles of HSF1 and NEDD4 in the brain of PITX3-IRES2-tTA/tetO- $\alpha-$ syn inducible double transgenic mouse expressing WT $\alpha$ syn under the PITX3 promoter predominantly expressed in midbrain dopaminergic neurons. TetO- $\alpha$-syn single transgenic mouse was used as a control to compare with double transgenic mice overexpressing $\alpha$-syn mostly in the midbrain area. By immunostaining, we confirmed somatic accumulation of $\alpha$-syn in tyrosine hydroxylase (TH)-positive dopaminergic neurons in midbrain substantia nigra of 12-month-old double transgenic mouse (Figure 3-3A). This abnormal accumulation of $\alpha$-syn in cell bodies was recently identified to be correlated with progression of neurodegeneration in $\alpha$-syn transgenic mice (Lin et al., 2009). Our results showed that the most reduction in HSF1 protein was detected in the midbrain samples of double transgenic mice where $\alpha$-syn was highly overexpressed, accompanied by strongly elevated NEDD4 in those compared with control (Figure 3-3B, C). However, the other three regions, hippocampus, cortex and cerebellum, did not show significant changes (Figure 3-3B). Interestingly, we observed two bands of HSF1 proteins only in mouse tissues; the upper band is higher than its predicted molecular weight of $82 \mathrm{kDa}$ which might be due to by post-translational events such as phosphorylation (Figure 3-3B). Although NEDD4 expressed primarily in the perinucleus of neurons in the age-matched control mice, we observed that NEDD4 was enormously induced in the soma of cells where $\alpha$-syn accumulated in the brain slices of $\alpha$-syn transgenic mice by immunohistochemistry (Figure 3-3C). While Lewy body in PD is primarily found in the midbrain substantia nigra and locus ceruleus, DLB is also categorized as $\alpha$-synucleinopathy present in the subcortical and

The review of literature provides an overview of the evolving significance of heart rate variability (HRV) as a surrogate measure of autonomic function. HRV is defined and a synthesis of its measurement methods and parameters given. Research 
Figure 3-3. Reciprocal NEDD4 and HSF1 Levels in Mouse and Human Tissue of $\alpha$-synucleinopathy.

(A) Somatic $\alpha$-syn overexpression (red) in the substantia nigra of $\alpha$-syn double transgenic mice. Green and yellow indicate TH-positive cells and those with somatic $\alpha$-syn aggregation, respectively. Scale bar: $10 \mu \mathrm{m}$. (B) (Top panels) Western blot analysis of the midbrain tissue of TetO- $\alpha$-syn single and $\alpha$-syn double transgenic mice (12 months of age). Quantification of HSF1/NEDD4 protein expression ( $* * * \mathrm{P}<0.001, * \mathrm{P}<0.05$, means $\pm \mathrm{SD}$ ). (Bottom panels) Western blots on four different areas of the brain (i.e. hippocampus, midbrain, cerebral cortex and cerebellum) with equal loading of total proteins. S, single transgenic mice (control); D, double transgenics ( $\alpha$-syn Tg). The red arrow indicates the predicted molecular weight of HSF1 (82 kDa). (C) Confocal microscopy of the substantia nigra from single and double $\alpha$-syn transgenic mice. Top panels: HSF1 (red); $\alpha$-syn (green). Bottom panels: NEDD4 (red); $\alpha$-syn (yellow). Scale bar: $10 \mu \mathrm{m}$ (top panels) and $2 \mu \mathrm{m}$ (bottom panels). (D) Western blot analysis of the inferior parietal lobes of human patient specimens with and without diffuse Lewy body disease (left panel). Immunohistochemistry of HSF1 (red) and $\alpha$-syn (green) (right panel). Scale bar: $10 \mu \mathrm{m}$. 
A
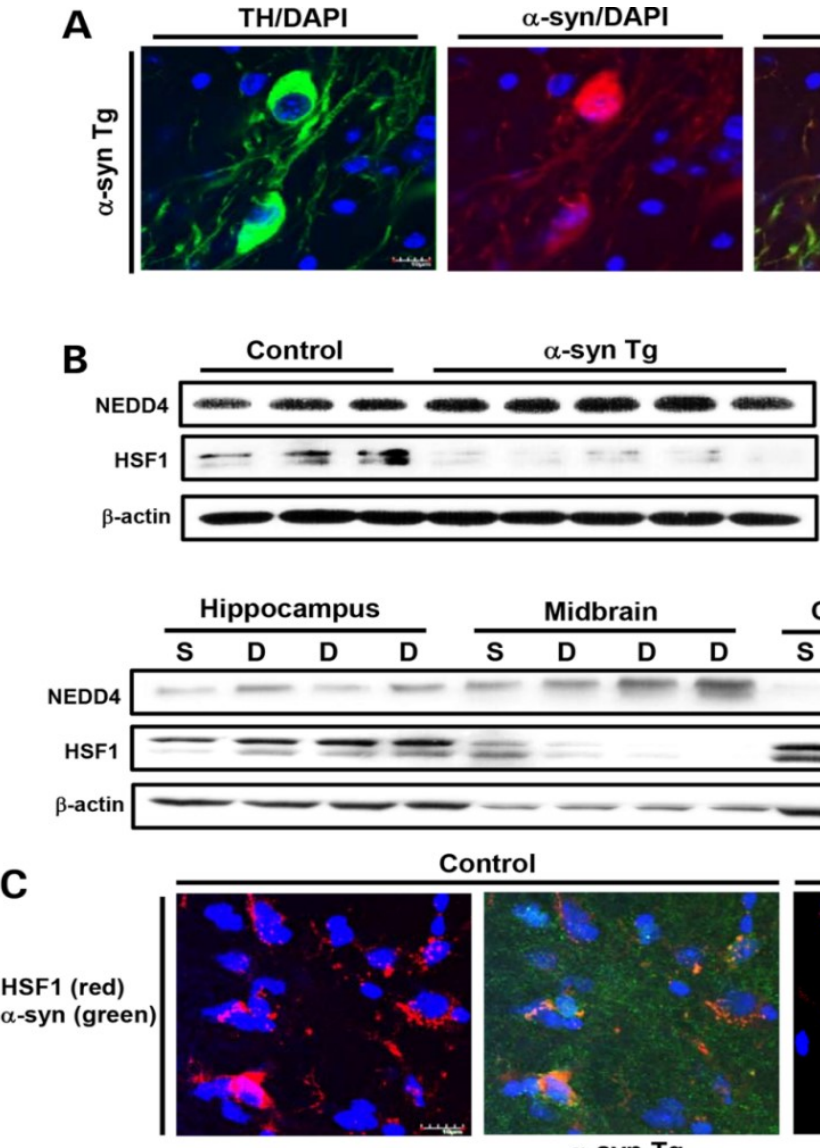

$\alpha$-syn Tg
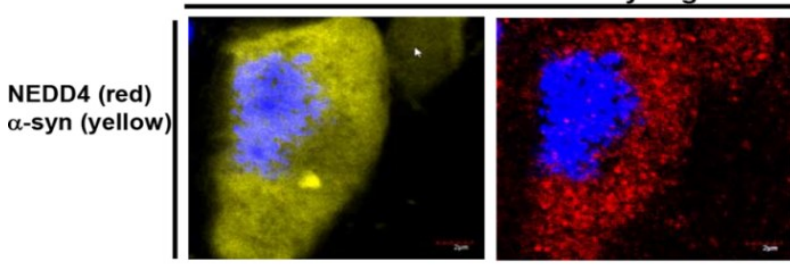

D

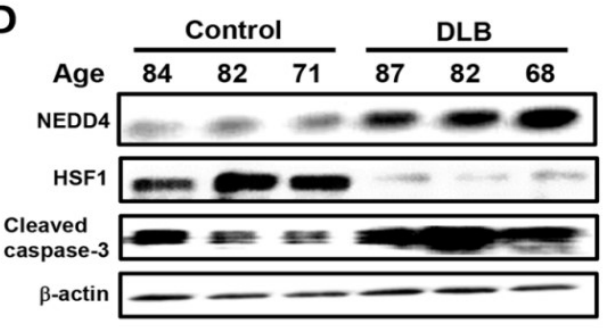

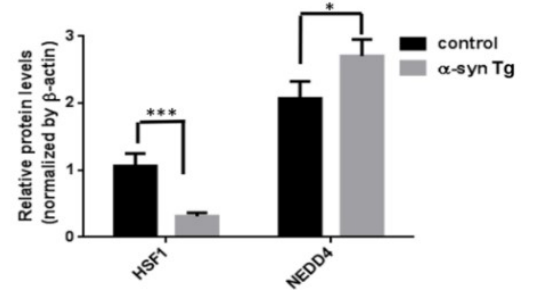
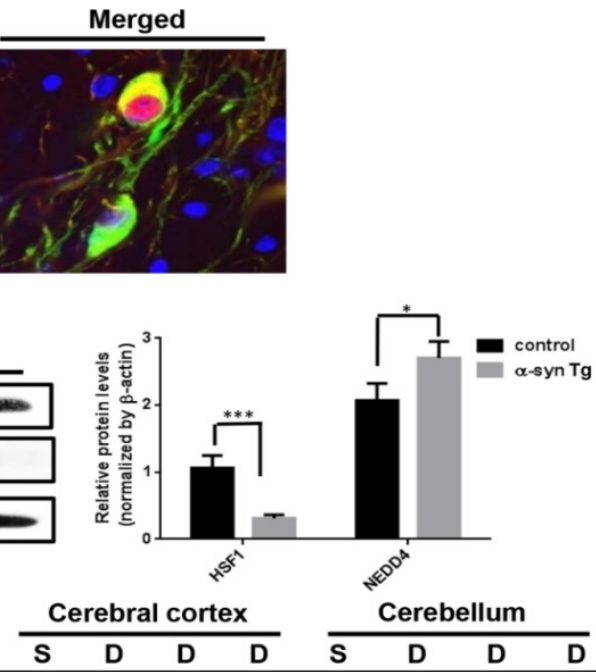

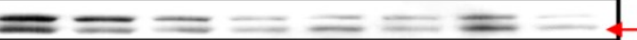

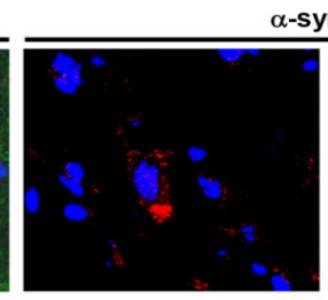

$\alpha$-syn Tg
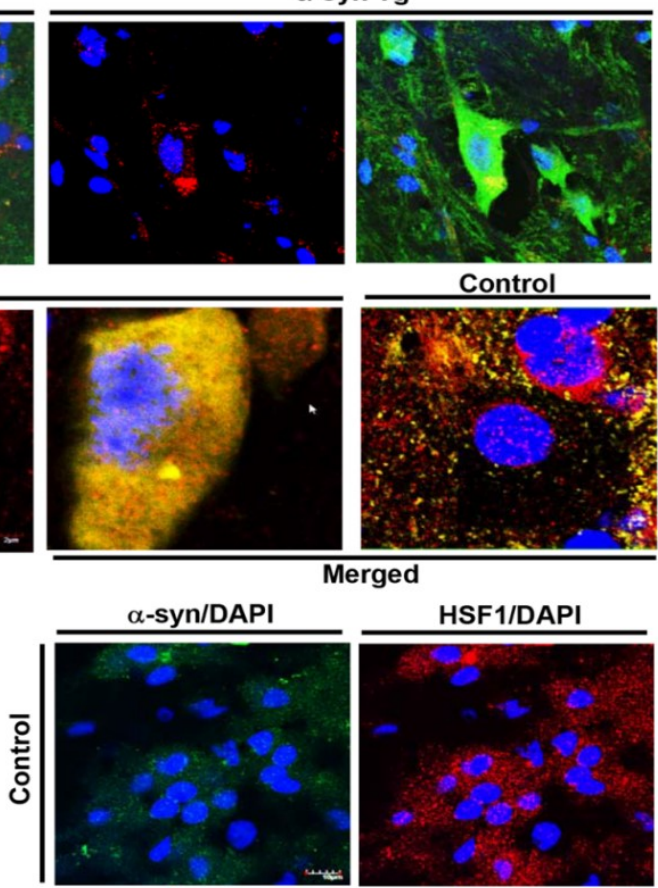

Control

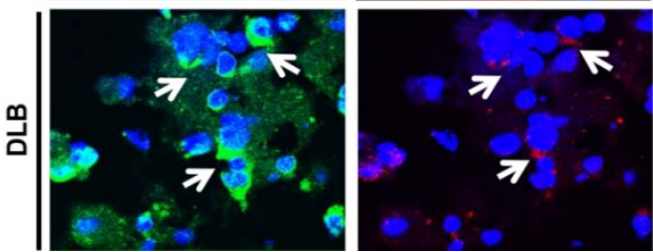


exploring cardiac and non-cardiac physical conditions, and psychosocial factors, cortical (frontotemporal) regions of the brain. We observed a striking decrease in HSF1 and NEDD4 upregulation within the inferior parietal lobes of human patients with DLB where Lewy body deposits were located (Figure 3-3D). Cleaved-caspase 3 as a marker of apoptosis was highly expressed in the individuals with reduced HSF1 (Figure 3-3D, left panel). Immunostaining of HSF1 showed a punctate pattern which colocalized with aggregated $\alpha$-syn, likely indicating stress granules (Figure 3-3D, right panel).

Since reciprocal expression levels of NEDD4 and HSF1 were found in mouse and human tissue of $\alpha$-synucleinopathy, we then sought in vivo evidence of $\alpha$-syn-induced HSF1 degradation. Six-month-old C57BL/6 mice were injected with AAV2 or AAV5CBA- $\alpha$-syn in the right substantia nigra and AAV-CBA-eGFP virus in the left side as control (Figure 3-4A). Remarkably, 3 weeks later, drastic HSF1 degradation was detected only in the right side of substantia nigra overexpressing $\alpha$-syn (Figure 3-4B, C), ac- companied by markedly increased NEDD4 which appeared to co-localize with $\alpha$-syn around perinucleus (Figure 3-4D). In addition to immunohistochemistry, 5-6 weeks after injection, the cortex and midbrain samples of two AAV2-CBA- $\alpha$-syn injected mice and one AAV5-CBA- $\alpha$-syn injected mouse were subjected to the western blot, which clearly demonstrated HSF1 degradation and NEDD4 elevation in the targeted midbrain area of AAV2- $\alpha$ - syn injected mice (Figure 3-4E).

\section{The Role of Acetylation Status in NEDD4-mediated HSF1 Degradation via Interplay with Ubiquitination}

In addition to ubiquitination, acetylation of HSF1 was remarkably elevated by A53T $\alpha$-syn aggregation in SH-SY5Y cells (Figure 3-5A). Resveratrol (RSV) has been shown to protect neurons against $\alpha$-syn toxicity in a SIRT1-dependent mechanism (Donmez et al., 2012). We investigated the effect of RSV on HSF1 levels as well as on its acetylation state. We found that $5 \mu \mathrm{M}$ of RSV was able to decrease $\alpha$-syn expression and increase HSF1 protein levels while decreasing HSF1 acetylation levels (Figure 3-5A). Most strikingly, NEDD4 overexpression resulted in reduced HSF1, correlating with highly acetylated HSF1. The RSV's effect on reducing HSF1 acetylation appeared to be dependent on its action on SIRT1 activation. Since co-transfection of A53T $\alpha$-syn with SIRT1 siRNA completely abolished its beneficial effect (Figure 3-5B). The tight regulation of HSF1 expression levels by SIRT1 was further confirmed by adding sirtuin inhibitors, sirtinol or nicotinamide (NAM), or co-transfection with a dominant-negative (DN) SIRT1 H355A defective in catalytic function (Figure 3-5E). Furthermore, SIRT1 WT overexpression prevented HSF1 degradation in A53T- transfected cells without altering HSF1 mRNA levels (Figure 3-5C). SIRT1 overexpression led to reduced interaction between HSF1 and NEDD4 as well as decreased polyubiquitination of HSF1 (Figure 3-5D). Taken together, HSF1 acetylation status appeared to be positively correlated with its ubiquitination but negatively with HSF1 protein stability under both basal and A53T-stressed conditions. 
Figure 3-4. HSF1 Degradation Induced by AAV-a-syn Viruses in Mouse Substantia Nigra.

Young adult C57BL/6 mice were injected with AAV- $\alpha$-syn or control eGFP viruses each side of SNc $(\mathrm{n}=4)$. (A-D) HSF1, NEDD4 and $\alpha$-syn levels were detected by immunohistochemistry. (A) Representative image of both sides of substantia nigra (SNc) is shown, each receiving AAV2-eGFP control (left side) or AAV2- $\alpha$-syn viruses (right side). White dotted boxes indicate the SNc regions. Scale bar: $500 \mu \mathrm{m}$. (B) Coronal brain slices were stained for HSF1 (red) and $\alpha$-syn (green); immunopositive signals are indicated by white arrows within the SNc regions. White circle shows that the $\alpha$-synpositive region is devoid of red HSF1 signal. Scale bar: $1 \mathrm{~mm}$. (C and D) The same brain slice from panel B was examined under confocal microscope, showing immunopositive signals of HSF1, NEDD4 and $\alpha$-syn with higher magnification. Scale bars: $10 \mu \mathrm{m}$ (C) and $5 \mu \mathrm{m}$ (D). (E) Western blot analysis of the cortex (unaffected area used as control) and midbrain where SNc is located of two AAV2- $\alpha$-syn injected mice and one AAV5- $\alpha$-syn injected mouse. L, left side injected with AAV-GFP virus; $R$, right side subjected to $A A V-\alpha$-syn virus injection. 

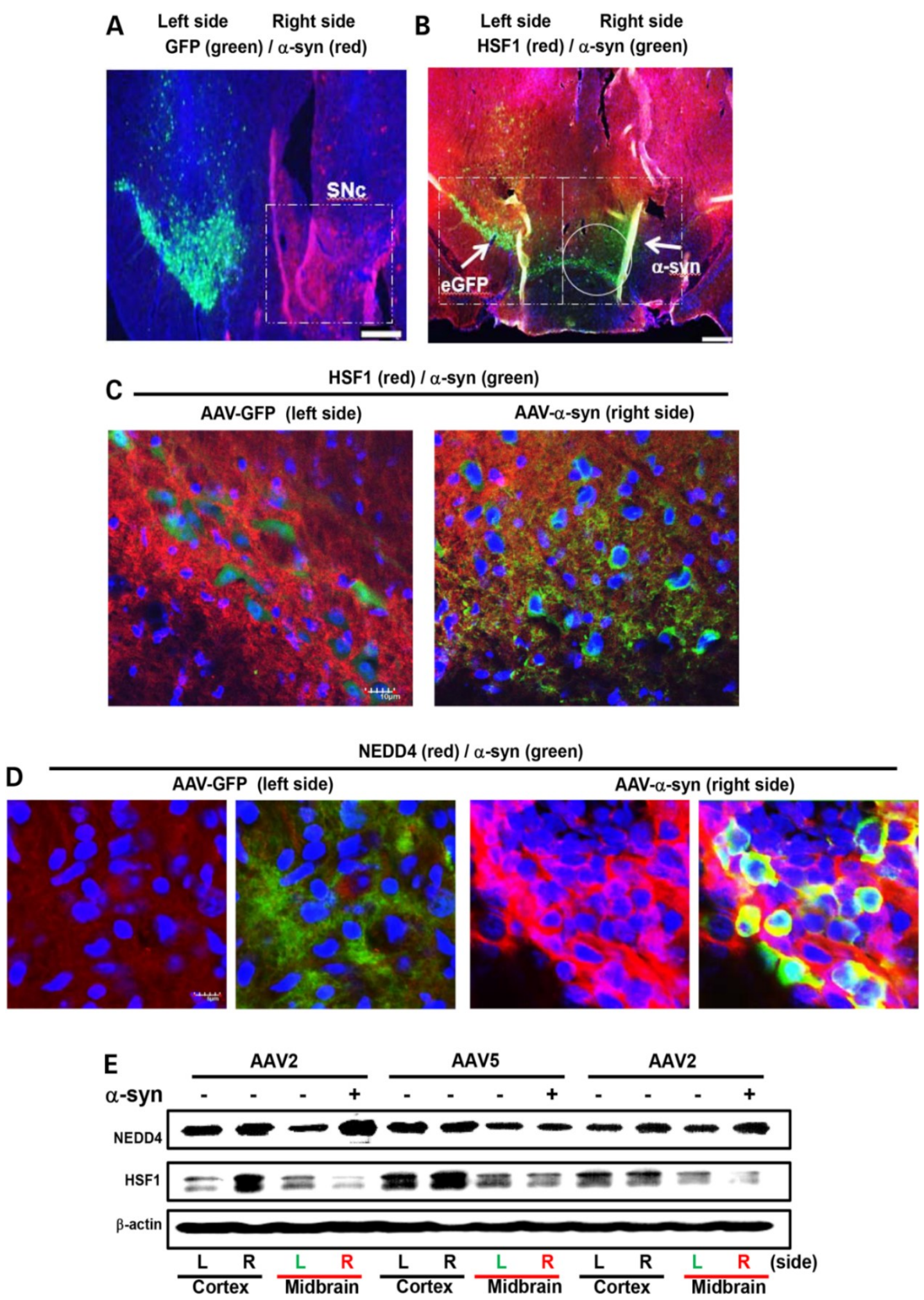
Figure 3-5. HSF1 Acetylation/Deacetylation and Ubiquitination.

(A and B) SH-SY5Y cells were co-transfected with NEDD4 WT plasmid or SIRT1 siRNA (B) for $48 \mathrm{~h}$ with or without co-treatment of RSV. After transfection and RSV treatment, cell lysates were analyzed by acetylation assay using immunoprecipitation and western blot. (C and D) Effects of SIRT1 WT overexpression on HSF1 deacetylation, ubiquitination and NEDD4 interaction with HSF1. (E) Effect of $5 \mathrm{~mm}$ NAM on HSF1 and $\alpha$-syn protein expression levels. Quantification of HSF1 protein levels normalized to $\beta$-actin in cells treated with $10 \mu \mathrm{M}$ sirtinol and $5 \mathrm{~mm} \mathrm{NAM}$, and overexpressing SIRT1 H355A ( $\mathrm{n}=3, * \mathrm{P}<0.05, * * * \mathrm{P}<0.001$, means $\pm \mathrm{SD}$ ). (F) Cells overexpressing A53T with or without SIRT1 WT were co-transfected with HSF1 WT or HSF1 K80Q or HSF1 $\mathrm{K} 80 \mathrm{R}$. The resulting cell lysates were subjected to in vivo ubiquitination assay. Endogenous HSF1 was pulled down by HSF1 antibody and HSF1-complex was subjected to immunoblot with anti-NEDD4 and anti-ubiquitin antibodies. In vivo ubiquitination assay in control cells without A53T mutation (right panel). 
A

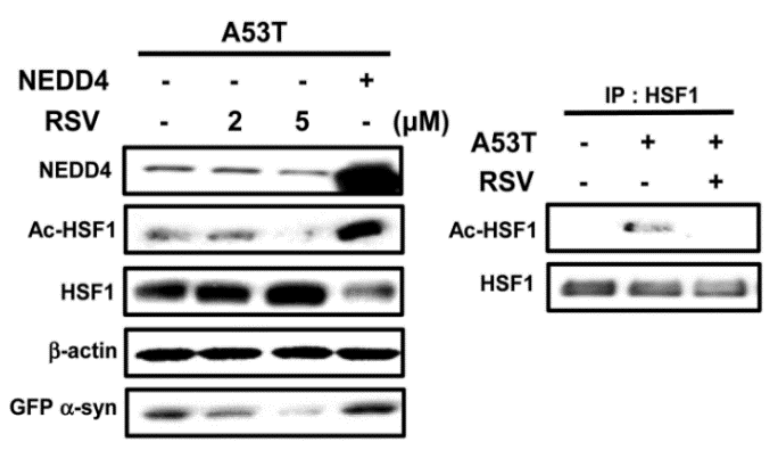

B

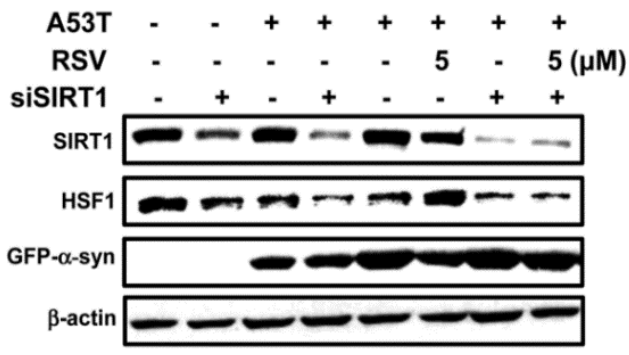

C
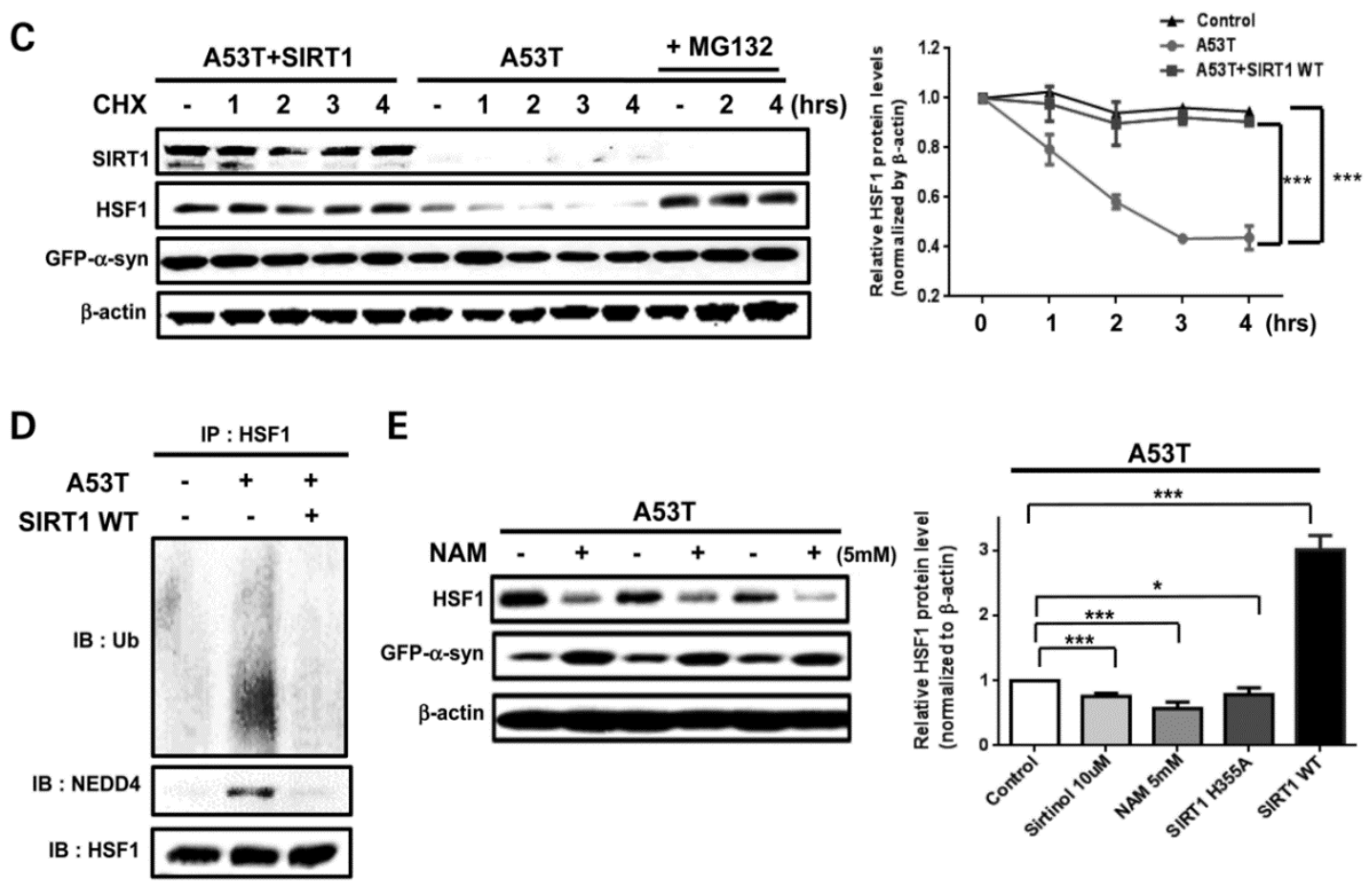

E
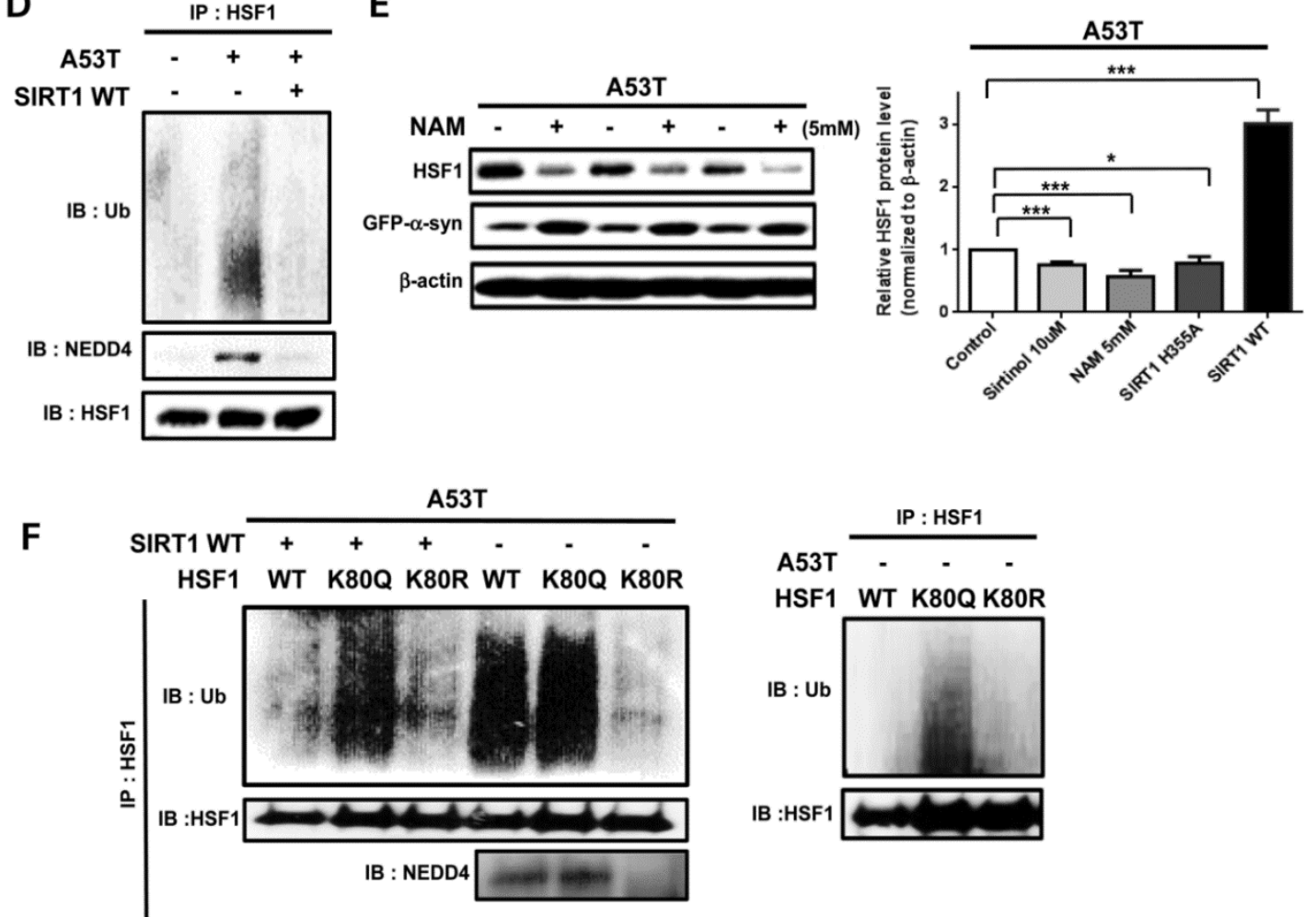
K80 is the lysine site located in the DNA-binding domain of HSF1 where its deacetylation was found to increase DNA-binding activity on the promoters of HSP genes (Westerheide et al., 2009). Therefore, it has been indirectly implicated as the major target by SIRT1 whose activation causes a delay in the attenuation of heat shock response (HSR). Given the positive role of SIRT1 in stabilizing HSF1 protein, we sought to determine if $\mathrm{K} 80$ is also responsible for the underlying mechanism. We replaced $\mathrm{K} 80$ with either glutamine (K80Q) to mimic acetylation or arginine (K80R) as nonacetylatable mutation. Results from the CHX experiment indicated that the extent of HSF1 degradation was minimized in A53T-transfected SH-SY5Y cells overexpressing K80R as compared with K80Q (data not shown). Accordingly, while HSF1 K80R was completely resistant to ubiquitination, HSF1 K80Q was highly mono- and polyubiquitinated than HSF1 WT was (Figure 3-5F). This tendency of HSF1 ubiquitination upon K80 residue was preserved in SIRT1-overexpressing A53T-transfected cells (Figure 3-5F). Similarly, under basal condition without A53T stress, only K80Q mutation caused HSF1 polyubiquitination (Figure 3-5F).

\section{Downregulation of NEDD4 or Overexpression of HSF1 or RSV Provides Neuroprotection against $\alpha$-synucleinopathy}

In support of the previously identified neuroprotective role of HSF1, we confirmed that overexpression of HSF1 (or HSF1 S303A, a constitutively activated form of HSF1) greatly reduced $\alpha$-syn toxicity as measured by $\alpha$-syn aggregation and reactive oxygen species (ROS) levels in A53T-trasnfected cells (Figure 3-6A, B). Furthermore, downregulation of NEDD4 (or C866S NEDD4) resulted in similarly outcomes

(Figure 3-6A, B, C, and D). On the contrary, NEDD4 overexpression led to increased caspase- 3 activation, despite decreased $\alpha$-syn aggregation from NEDD4 overexpression in cells overexpressing A53T $\alpha$-syn (Figure 3-6A, B, C, and D). This is consistent with the results from western blot suggesting that the pro-apoptotic Bax was rather increased although $\alpha$-syn accumulation was reduced upon NEDD4 overexpression (Figure 3-2E). Finally, RSV was proven to be protective as reported previously (Figure 3-6A, B, C, and D).

\section{Summary}

Here we have provided compelling and novel in vitro and in vivo evidence that proteotoxic stresses, as exemplified by $\alpha$-synucleinopathy, induce rapid and sustained HSF1 protein degradation mediated by the E3 ligase NEDD4 through UPS. First, we discover aberrant HSF1 degradation by aggregated $\alpha$-synuclein (or $\alpha$-synuclein-induced proteotoxic stress) in transfected neuroblastoma cells.

We show here that both WT and A53T mutant $\alpha$-syn caused HSF1 protein degradation to varying degrees: while A53T $\alpha$-syn-induced HSF1 lost in both cytosolic and nuclear compartments, WT $\alpha$-syn overexpression appeared to only induce loss of nuclear HSF1. Whether nuclear HSF1 is more vulnerable to proteotoxic stress remains 
Figure 3-6. Effects of Down-regulated NEDD4 or Overexpressed HSF1 or RSV on $\alpha$-syn Toxicity.

(A and B) ROS production was determined by DHE staining (red) for different groups of cells. Green indicates GFP-A53T $\alpha$-syn expression. A53T $\alpha$-syn transfected SH-SY5Y cells were co-transfected with NEDD4 WT, NEDD4 C866S or HSF1 S303A (constitutively activated) or treated with RSV $50 \mu \mathrm{M}$. Scale bar: $30 \mu \mathrm{m}$ (A). Graph indicates the percentage of fluorescence intensity per 100 cells (B). (C and D) Apoptotic cells identified by positive cleaved caspse-3 staining (red) with DAPI nuclear staining (blue). A53T $\alpha$-syn transfected SH-SY5Y cells were co-transfected with NEDD4 WT or NEDD4 siRNA, or treated with RSV $20 \mu \mathrm{M}$. Quantification of cleaved-caspase 3 stained nucleus for each group, represented as percentage on the graph (D). Scale bar: $30 \mu \mathrm{m}(\mathrm{B}$ and $\mathrm{D}$ graphs, $\mathrm{n}=4, * \mathrm{P}<0.05, * * \mathrm{P}<0.01, * * * \mathrm{P}<0.001$, means $\pm \mathrm{SD}$ ). (E and $\mathrm{F}) \mathrm{The}$ effect of WT and different mutant forms of HSF1 overexpression on A53T $\alpha$-syn-induced apoptosis was determined by positive cleaved caspase-3 signals. (E) Representative image of GFP-A53T $\alpha$-syn (top panels) and cleaved caspase- 3 staining (bottom panels) in transfected groups of cells. Red: cleaved caspase-3, Blue: DAPI, Green: GFP-A53T $\alpha-$ syn. Scale bar: $2 \mathrm{~mm}$. (F) Quantitative analysis of expression of A53T $\alpha$-syn and cleavedcaspase 3, showing significant difference in the number of GFP and activated-caspase 3 positive cells compared with the single (A53T) transfection group $(\mathrm{n}=3, * \mathrm{P}<0.05, * * \mathrm{P}$ $<0.01, * * * \mathrm{P}<0.001$, means $\pm \mathrm{SD})$. Around 100 cells were counted for each transfected group. (G) Western blot representing change in expression levels of inducible Hsp70, GFP- $\alpha$-syn, and Bax as a result of overexpression of WT HSF1 and S303A/K80R/ K80Q mutant forms of HSF1, as well as RSV $20 \mu \mathrm{M}$ treatment in A53T $\alpha$-syn-transfected cells. 
A

GFP- $\alpha$-syn A53T (Green) / anti-ROS (Red, DHE, dihydroethidium)

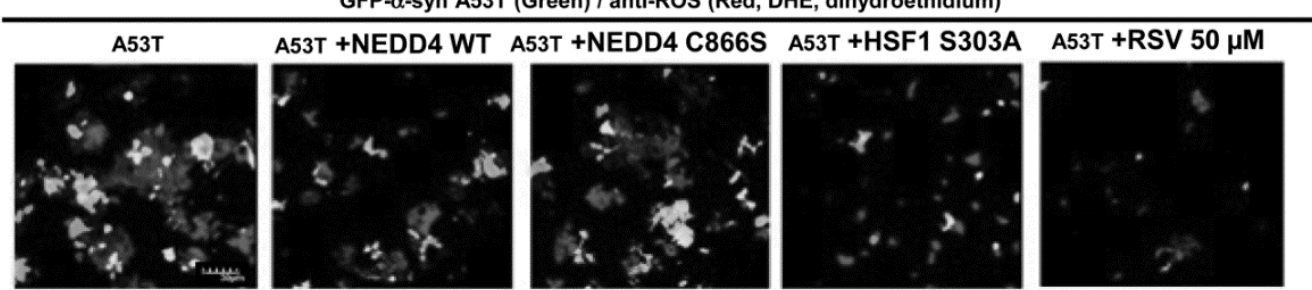

B

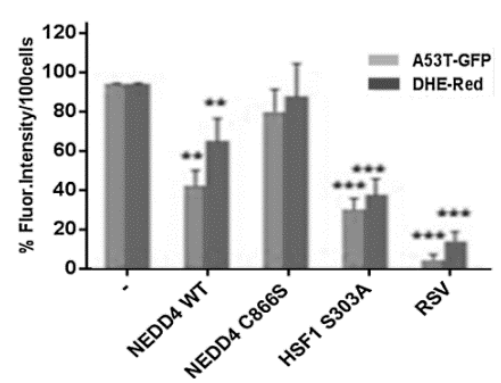

C

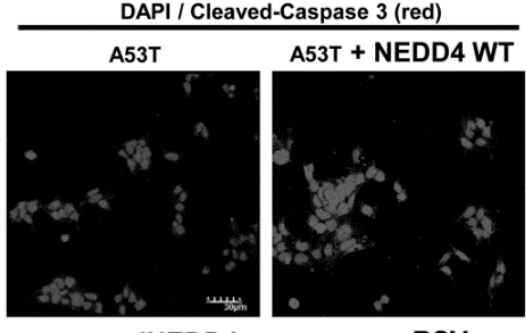

D
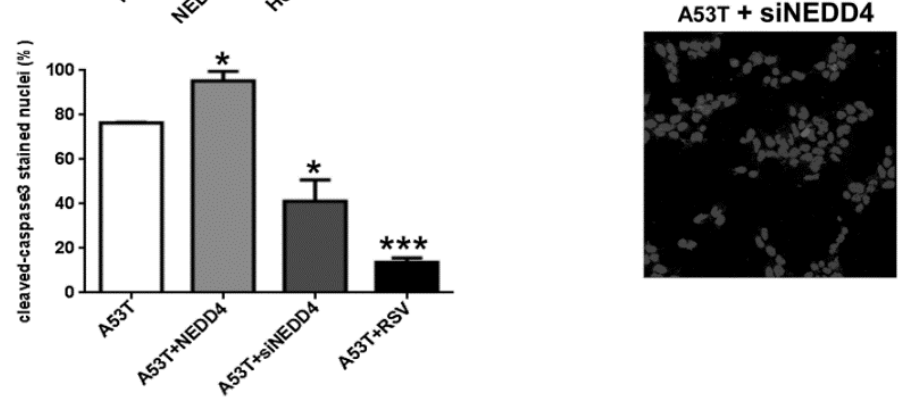

A53T + RSV
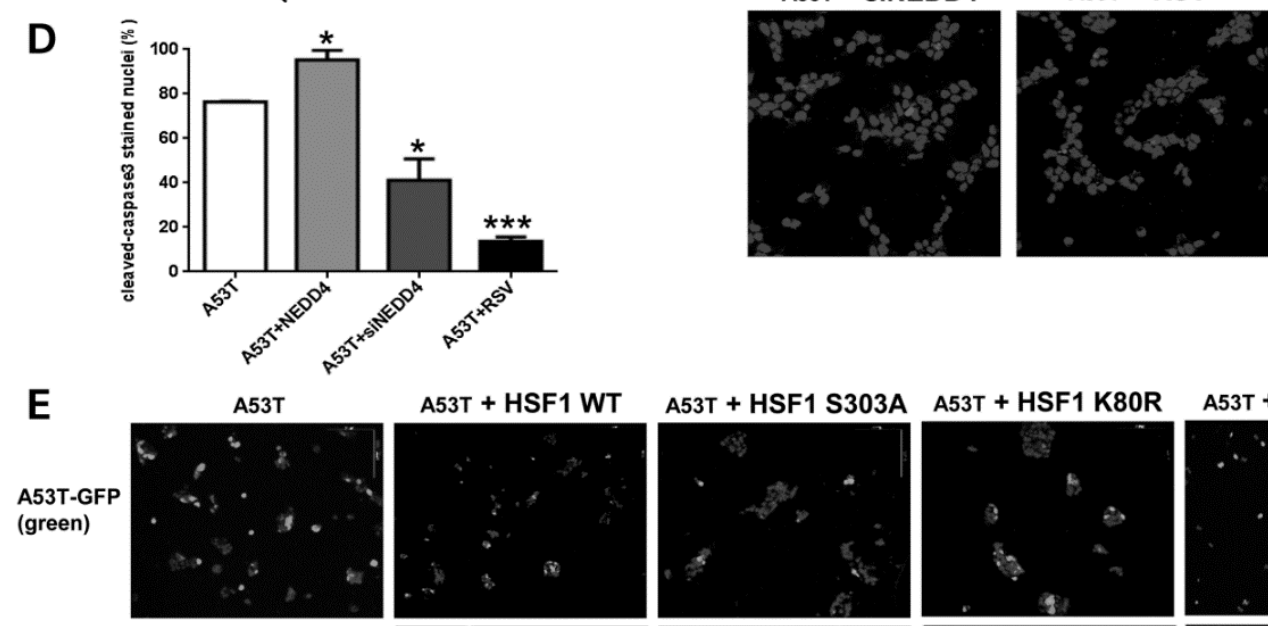

A53T + HSF1 WT
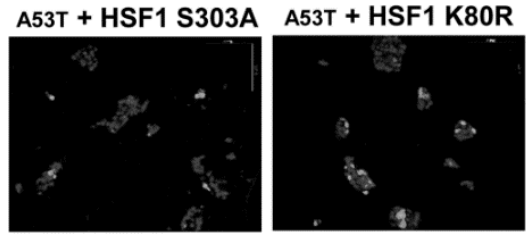

A53T + HSF1 K80Q
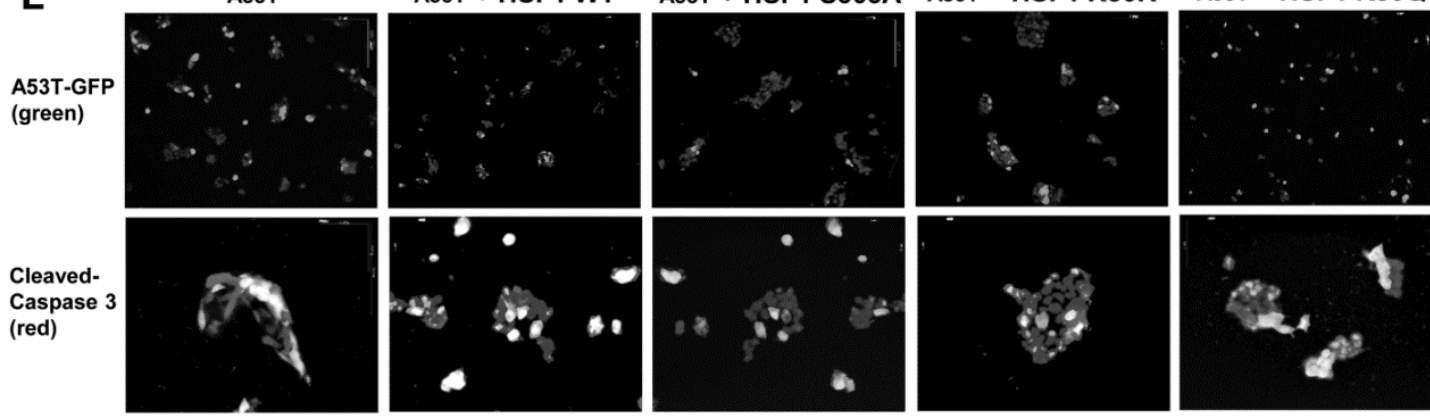

$\mathbf{F}$

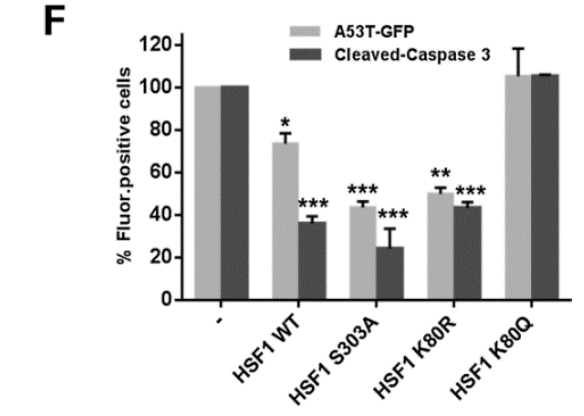

G

A53T
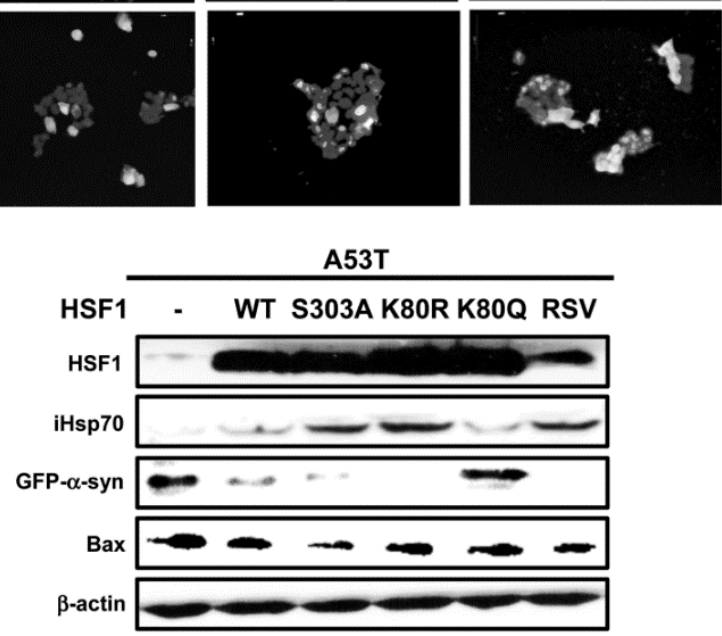
unclear. Importantly, HSF1 dysregulation via $\alpha$-synuclein was confirmed by in vivo assessment of mouse and in situ studies of human specimens with $\alpha$-synucleinopathy. We demonstrate that elevated NEDD4 is implicated as the responsible ubiquitin E3 ligase for HSF1 degradation through UPS. Furthermore, pharmacologically induced SIRT1mediated deacetylation can attenuate aberrant NEDD4-mediated HSF1 degradation. Indeed, we define the acetylation status of the Lys 80 residue located in the DNA-binding domain of HSF1 as a critical factor in modulating HSF1 protein stability in addition to its previously identified role in the transcriptional activity. We demonstrate that the same Lys 80 acetylation state directly correlates with the ubiquitination levels and the stability of HSF1. The underlying mechanism is unclear. Our study has demonstrated the potential significance of SIRT1-mediated deacetylation in HSF1 stability, adding to the growing body of evidence of sirtuins in the regulation of proteostasis (Sampalo-Marques et al., 2015). Fully delineating the underlying mechanism of SIRT1-mediated proteostasis will facilitate development of this promising strategy.

Together with the finding that preserving HSF1 can alleviate $\alpha$-synuclein toxicity, this study strongly suggests that aberrant HSF1 degradation is a key neurodegenerative mechanism underlying $\alpha$-synucleinopathy. This work suggests that aberrant HSF1 protein degradation may represent a common and important key molecular mechanism underlying neurodegeneration. 


\section{CHAPTER 4. THE ROLE OF HSF1 PROTEIN REGULATION IN TAUOPATHY}

\section{Introduction}

Neurofibrillary tangles (NFTs) of phosphorylated tau aggregates and senile plaques of amyloid beta $(A \beta)$ are the pathological hallmarks of Alzheimer's disease (AD) patients. $A \beta$ toxicity has been known to contribute to synaptic loss and cognitive impairment, the mechanism of which appears to be tau-dependent (Mukaetova-Ladinska et al., 2000, Nelson et al., 2010, Nijholt et al., 2012). However, NFTs, not amyloid plaques, have been identified to correlate best with the severity of dementia (Arriagada et al., 1992, Mocanu et al., 2008). NFTs exist primarily inside the cell, and have been associated with cellular stress responses (Butler et al., 1986, de Calignon et al., 2012, Shipton et al., 2011). Such response systems include unfolded protein response (UPR) initiated in the endoplasmic reticulum (ER) and cytoplasmic heat shock response initiated by heat shock factor 1 (HSF1) activation. Both pathways involve transcriptional activation of the stress-response genes. A key feature of heat shock response is to induce a set of molecular chaperone proteins such as Hsp 70 whose function is to correct protein folding in response to numerous cellular stresses. Likewise, UPR is initially triggered as an adaptive response to disturbances in ER homeostasis. In this sense, HSP70 a5 (BiP/GRP78), a major ER chaperone Hsp70, has been found to attenuate ER stress by activating sensors of transmembrane ER stress, such as PERK, via direct binding (Bertolotti et al., 2000). However, sustained chronic UPR activation as a result of unresolved ER stress can eventually trigger cell death by inducing pro-apoptotic proteins such as $\mathrm{C} / \mathrm{EBP}$ homologous protein (CHOP) primarily through the PERK/eIF2 $\alpha / \mathrm{ATF} 4$ pathway (Gorbatyuk et al., 2013, Roberson et al., 2007, Spillantini et al., 1997). A close correlation between ER stress markers and NFTs has been consistently reported in human tauopathies such as AD and frontotemporal dementia (de Calignon et al., 2012, Mori et al., 1992, Rapoport et al., 2002). Ho et al., 2012 has shown that ER stress can cause tau hyperphosphorylation in primary cultured neurons (Guettouche et al., 2005). However, it is unknown whether and how ER stress causes tau phosphorylation, and vice versa. While these two stress systems (i.e. heat shock response, UPR) have been originally considered to be individually triggered by distinct stressors, recent studies have begun to highlight the importance of heat shock responses in relieving ER stress in non-neuronal cells (Dou et al., 2003, haggerty et al., 2011, Hou et al., 2014, Schmitt et al.,2012). Nevertheless, how ER stress affects HSF1-mediated stress response is poorly understood, particularly in the contexts of neurons and tauopathy.

We recently identified aberrant HSF1 degradation via ubiquitin proteasome system as an important mechanism underlying synucleinopathy (Homma et al., 2007). Synucleinopathy has been suggested to be pathogenetically linked with tauopathy as reflected by their frequent co-occurrence in neurodegenerative diseases and the synergistic interaction of tau and synuclein (Drexler et al., 2009, Giasson et al., 2003, Lee et al., 2010). Tauopathy is characterized by a build-up of tau aggregates, and thus the notion that HSF1 could also be degraded by tau aggregation stands as an intriguing 
possibility. In an attempt to understand human AD tau pathology, experimental murine tauopathy has been generated by introducing mutations in the human tau gene causing frontotemporal dementia and parkinsonism linked to chromosome 17 (FTDP-17) such as P301L and $\triangle K 280$ (Hunsberger et al., 2015, Min et al., 2010). Presence of UPR activation in the tau transgenic mouse models has not been clearly defined. Spatara et al., 2010 reported that PS19 mice harboring human tau P301S variant did not show any signs of UPR activation (Ramsden et al., 2005), whereas Abisambra et al., 2013 provided some evidence of UPR activation in rTg4510 mice overexpressing human tau carrying the P301L mutation (Abosambra et al., 2013). Relative to the P301L missense mutation, the Tau $\Delta \mathrm{K} 280$ mutation has been known to aggregate faster than any other single missense mutation (Resende et al., 2008). Tau $\Delta \mathrm{K} 280$ mutation has never been discussed in terms of its involvement of UPR activation. Here, we examined the impact of ER stressinduced UPR activation on HSF1 protein and vice versa in promoting aberrant tau pathology in AD. We investigated how UPR effector proteins such as CHOP and HSP70 a5 (BiP/GRP78) regulate HSF1 to potentiate a vicious cycle active in the cellular tauopathy model (N2a-TauRD $\Delta$ K280). Our work highlights that HSF1 loss may constitute a mechanistic connection between ER stress and tau hyperphosphorylation, especially in stress-vulnerable neuronal populations such as the hippocampus or aged brain.

\section{Results}

\section{Loss of HSF1 Protein Is an Early Event That Precedes NFTs Formation in AD, Which Is Further Exacerbated in the Presence of PERK (or UPR) Activation in the Brains of Mouse and Human Tauopathy}

To determine whether expression of HSF1 protein and UPR marker proteins was altered in the mouse tauopathy models, we looked at the brains of PS19 (tau P301S) mouse and rTg(tauP301L)4510 mouse overexpressing P301S and P301L mutant tau, respectively. It was previously reported that synaptic function was impaired in 3 monthold PS19 mouse before NFTs that consist of insoluble hyperphosphorylated tau developed at 6 months of age (Yoshiyama et al., 2007). In the brain of 4 month-old PS19 mouse, we found that overexpressed mutant tau was not hyperphosphorylated at Ser202/Thr205 (p-Tau, detected using the AT8 antibody) (Figure 4-1A, $\mathrm{P}<0.01, \mathrm{n}=5$ ). Upon activation of the UPR signal, PERK is phosphorylated at Thr980 (p-PERK). While PERK was not activated, about $30 \%$ of HSF1 protein was lost in the brain of PS19 mouse before tau hyperphosphorylation at a presumably later stage (Figure 4-1A, $\mathrm{P}<$ $0.05, \mathrm{n}=5$ ). Insoluble tau filaments were previously detected in 4 month-old $\mathrm{rTg} 4510$ mouse (Ramsden et al., 2005). We observed that overexpressed mutant tau was highly phosphorylated in the brain of rTg4510 mouse, about 2.7-fold increase in the levels of pTau normalized to total tau protein (Tau46), at 4 months of the same age with PS19 mouse above (Figure 4-1B, $\mathrm{P}<0.001, \mathrm{n}=5$ ). Activated PERK causes phosphorylation on Ser51 of the $\alpha$ subunit of eukaryotic translation initiation factor 2 (p-eIF2 $\alpha$ ). Together 


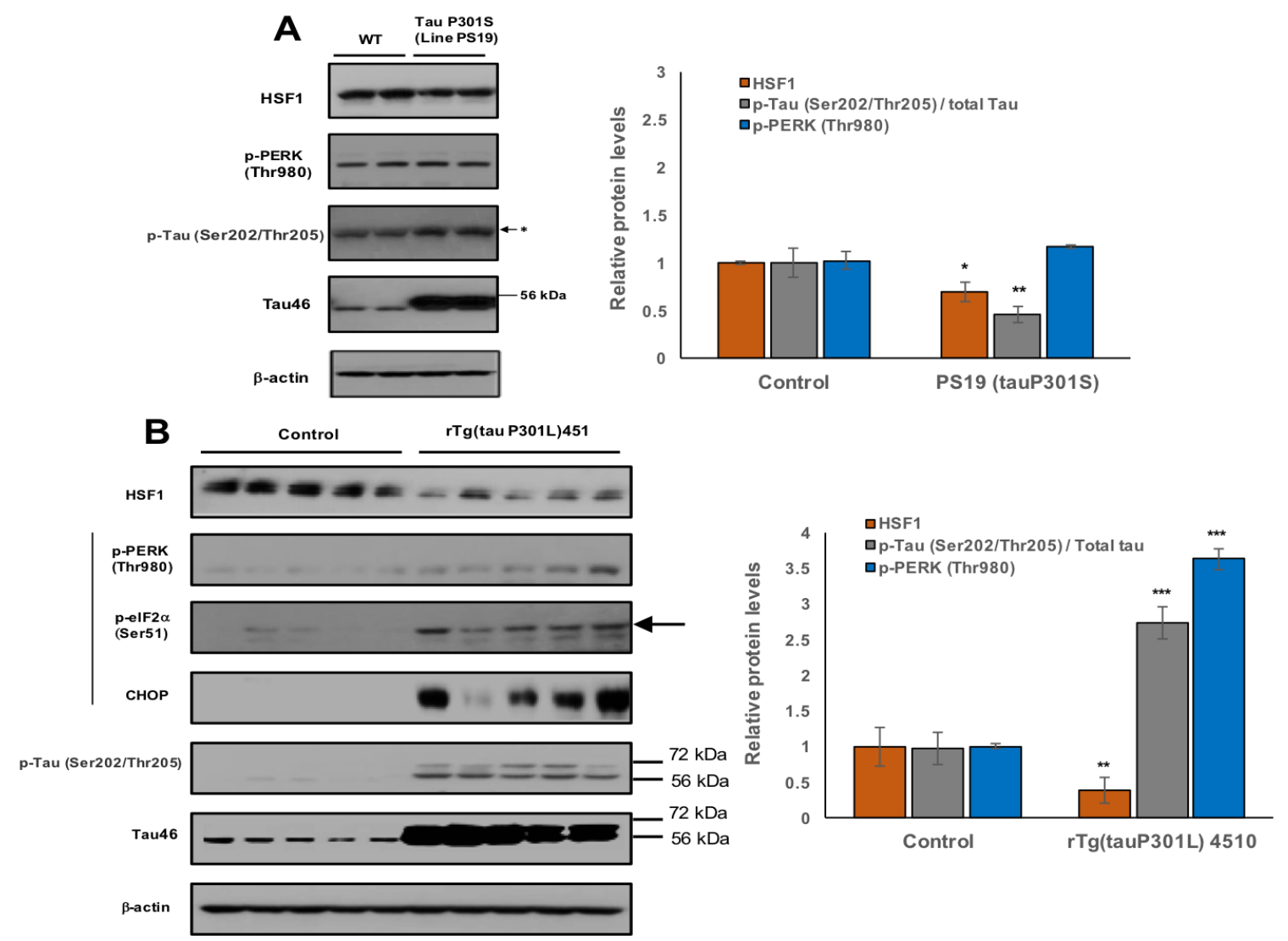

Figure 4-1. HSF1 Protein Loss and Chronic UPR Activation in the Brain of rTg(tauP301L)4510, and Minor Loss of HSF1 Protein in the Brain of PS19 (tauP301S).

(A) Expression levels of HSF1, p-PERK (Thr980), total Tau (Tau46), and p-Tau (Ser202/Thr205) in PS19 (tauP301S) and WT at 4 months of age were determined by western blot. *: $\sim 68 \mathrm{kDa}$ tau isoform. (B) HSF1 loss and UPR activation in the brains of rTg(tauP301L)4510 at 4 months of age. Expression levels of HSF1, p-PERK (Thr980), peIF2 $\alpha$ (Ser51), total Tau (Tau46) and p-Tau (Ser202/Thr205) in rTg4510 brain were determined by western blot (A vertical line highlights UPR marker proteins, herein after). Arrow indicates the band of p-eIF $2 \alpha$ at $38 \mathrm{kDa}$, its original size. Tau46 antibody is expected to detect total Tau at 50-80 kDa. Control mice used are (from left to right) single transgenic (Tg) CaMKII-tTA, single Tg TRE-tau, non-Tg, non-Tg, and single Tg TRE-tau. Quantification of p-PERK, HSF1 (normalized to $\beta$-actin) and p-Tau (Ser202/Thr205) protein (normalized to total Tau protein (Tau46)) in the whole brain samples of PS19 (A), rTg4510 (B) and their control (means \pm SEM, $* P<0.05, * * P$ $<0.01,{ }^{*} * P<0.001$, comparing to control, $\mathrm{n}=5$ (A), $\mathrm{n}=10(\mathrm{~B})$ ). 
with p-PERK and p-eIF2 $\alpha$ elevation, increased expression of pro-apoptotic protein CHOP suggested later apoptotic stage of UPR chronically activated in the brain of rTg4510 mouse (Figure 4-1B, p-PERK, $\mathrm{P}<0.001, \mathrm{n}=5$ ) (Wang et al., 1996, Zinszner et al., 1998). We detected a dramatic loss of HSF1 expression levels, about $60 \%$ reduction in rTg4510 mouse brain in which PERK (or UPR) was activated (Figure 4-1B, P $<0.01$, $\mathrm{n}=5)$.

The Braak NFT staging system is used to classify the anatomical distribution of AD-type NFTs according to stages (I/II, entorhinal-; III/IV, limbic-; and V/VI, neocortical-stage) (Braak et al., 1991). To identify the sequential relationship of molecular events in AD brain, we determined the expression levels of HSF1 protein and UPR marker proteins in the frontal lobes of 19 human postmortem brain specimen, spanning different Braak NFT stages. Distribution of HSF1 protein mostly in the cytoplasm suggested its inactivation in aged human brain since HSF1 is a nuclear transcriptional factor likely to be located in the nucleus when it is activated (Figure 4-2A). More importantly, we did observe gradually decreased expression of HSF1 protein that occurred even before formation of NFTs composed of hyperphosphorylated tau (Figure 4-2A, B, and C). While PERK activates eIF2 $\alpha$ during UPR activation upon ER stress, other serine kinases such as general control nonderepressible 2 (GCN2) can also phosphorylate eIF2 $\alpha$ in response to other stresses including amino acid deprivation. In incipient AD stages III/IV (before the frontal cortex starts to form NFTs), we observed about $58 \%$ reduction in HSF1 protein and slightly increased p-eIF2 $\alpha$ without PERK activation (Figure 4-2B, C). UPR was activated in later stage $\mathrm{V}$ patients diagnosed with early onset $\mathrm{AD}(\mathrm{EAD})$ and $\mathrm{AD}$ patients in stage $\mathrm{VI}$, as reflected by marked hyperactivation of PERK and eIF2 $\alpha$ (Figure 4-2B, C). About $59 \%$ of total HSF1 protein in Stages III/IV was further lost in stages V/VI when UPR was activated. It should be noted that stage $\mathrm{V}$ patients diagnosed with EAD demonstrated significantly greater $\mathrm{p}$-PERK and less HSF1 protein expression when compared to AD patients in stage V (Figure 4-2B). Taken together, these results identify that a dramatic loss of HSF 1 protein is an early and progressive event that may precede PERK activation and NFTs formation in murine tauopathy and human AD.

\section{Haploinsufficiency of HSF1 Leads to Chronic PERK Activation and Causes Aberrantly Phosphorylated Tau Aggregation and Its Mislocalization in the Aged Mouse Hippocampus}

Next, we investigated if HSF1 protein loss altered the expression levels of UPR marker proteins and tau phosphorylation in the brain of HSF1 haploinsufficient mouse (HSF1+/-). Phosphorylation at Thr212/Ser214 (p-Tau, detected using the AT100 antibody) was not detected in the hippocampus of 2 month-old HSF1+/- and WT (Figure 4-3A). However, PERK and CHOP protein were highly activated in the hippocampus of 9 month-old HSF1+/-, although p-eIF2 $\alpha$ was not elevated (Figure 4-3B, p-PERK (Thr980), $\mathrm{P}<0.001$; CHOP, $\mathrm{P}<0.01$, comparing to 9 month-old WT hippocampus, $\mathrm{n}=6$ ). By immunohistochemistry, we found that activated PERK ( $\mathrm{p}$ - 

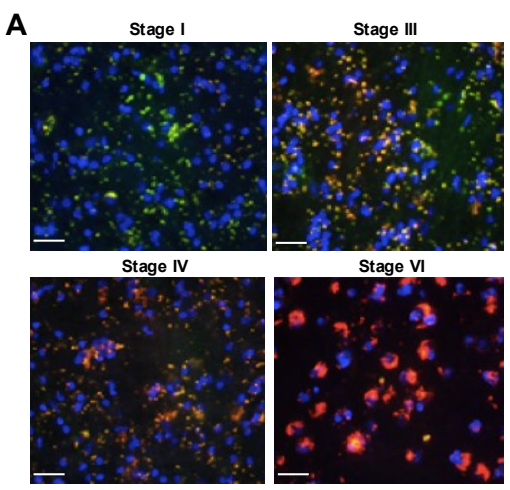

B
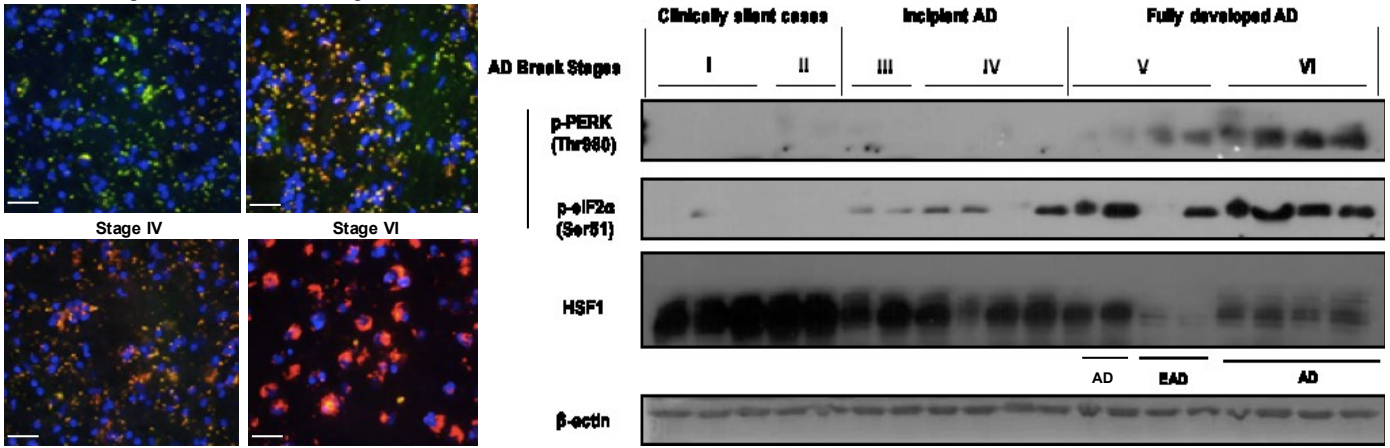

(Sarb1)

HSF1

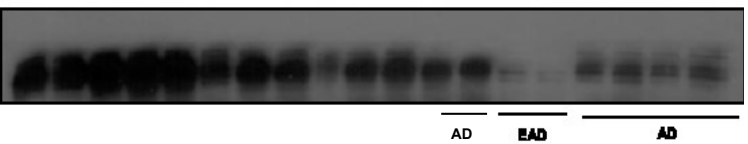

Bergtin
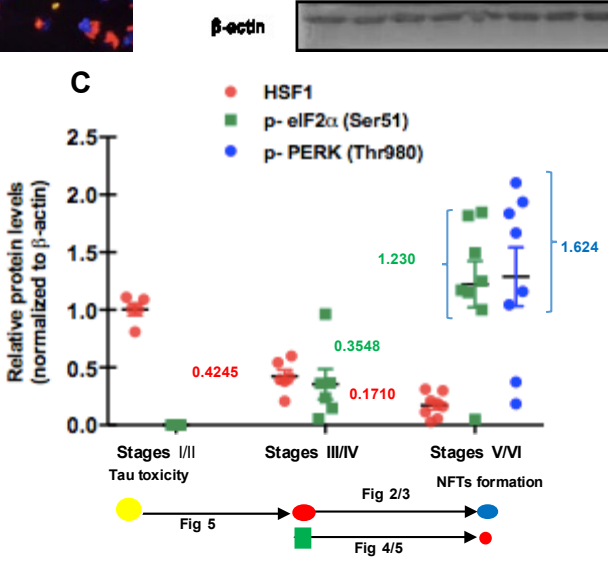

Figure 4-2. HSF1 Loss and UPR Activation in the Frontal Cortex of Human Brain at Different Stages of Alzheimer's Disease Progression (Braak Staging).

(A) Tau aggregation and a dramatic loss of HSF1 protein in human AD brain of Braak stage VI. Phosphorylated tau (p-Tau (Ser202/Thr205), detected by AT8 antibody), red) and HSF1 protein expression (green) in four different Braak stages (Braak stage I, III, IV, VI) were visualized by immunostaining. Scale bar: $1 \mathrm{~mm}$. (B) Protein lysates from frontal cortex of human brain at various Braak I-VI stages were subjected to western blot. EAD: early-onset Alzheimer's disease (AD). (C) Quantitative measurement of protein levels of p-PERK (blue), p-eIF2 $\alpha$ (green), and HSF1 (red) normalized to $\beta$-actin in human brain samples of distinct Braak stages (Braak stages I/II (Clinically silent cases); III/IV (Incipient AD); V/VI (Fully developed AD)). Error bars represent standard error of mean (SEM). Means indicated in the graph. The effect of left on right on each arrow is discussed in the following figures indicated 


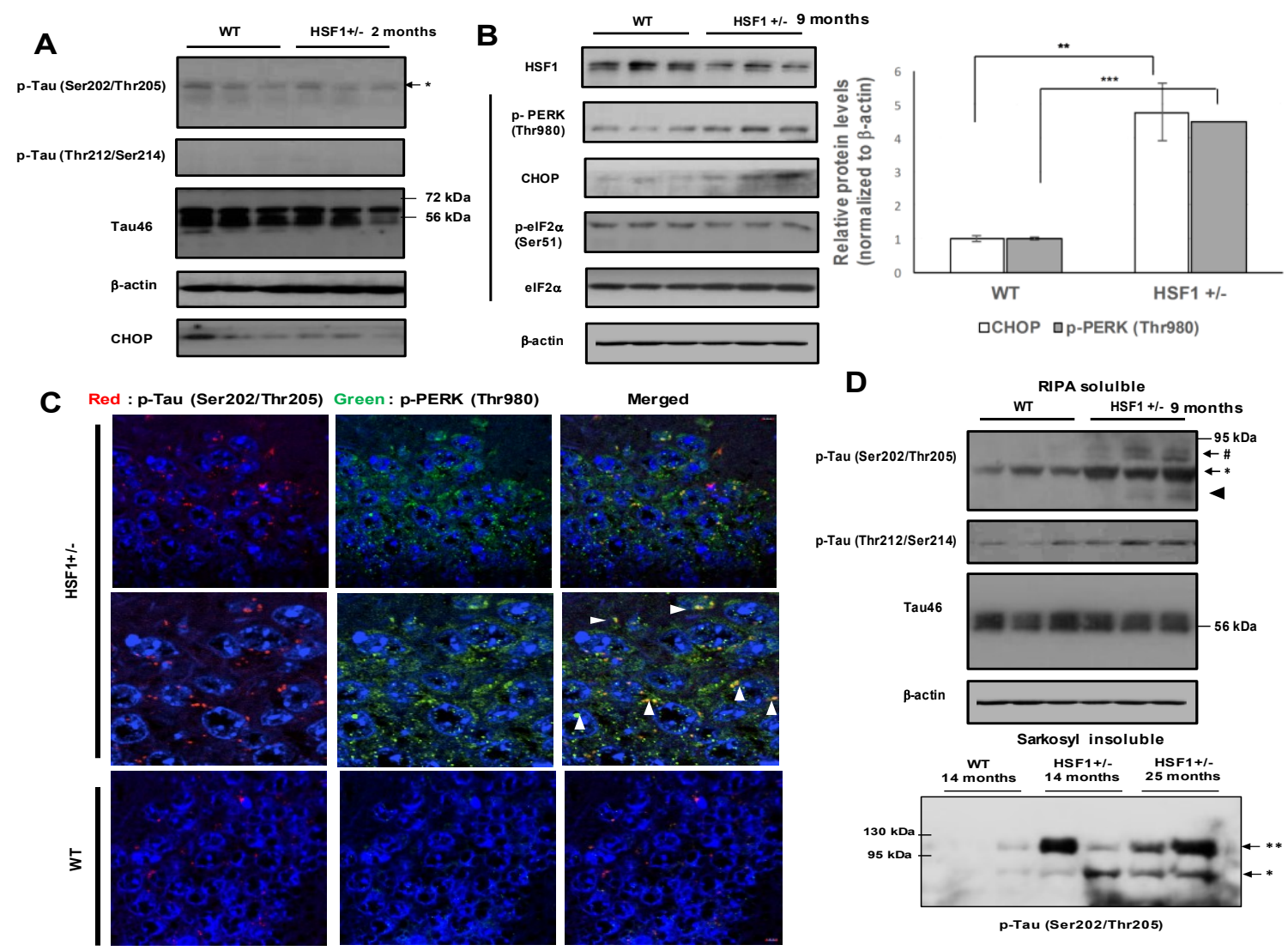

Figure 4-3. Chronic UPR Activation and Aberrantly Hyperphosphorylated Tau Aggregation in the Hippocampus of Aged HSF1 Haploinsufficient Mouse.

$(\mathrm{A}, \mathrm{B}, \mathrm{D})$ Western blot assays to examine protein expression levels of total tau (Tau46), pTau (at Ser202/Thr205 and Thr212/Ser214) and UPR marker proteins (p-PERK, p-eIF2 $\alpha$, eIF2 $\alpha$, and CHOP) in the hippocampus of WT and HSF1+/- at 2 months of age (A) and hippocampus of WT and HSF1+/- at 9 months of age (B, D). (B) Increased p-PERK (Thr980) and CHOP protein expression in the hippocampus of 9 month-old HSF1 heterozygous knock-out mice (HSF1+/-). Relative protein levels of CHOP and p-PERK (Thr980) normalized to $\beta$-actin in WT and HSF1 +/- at $\sim 9$ months of age are presented in the graph (right, means $\pm \mathrm{SEM}, * * P<0.01, * * * P<0.001, \mathrm{n}=6$ ). Western blots of RIPA-soluble tau (A and D) and sarkosyl-insoluble tau aggregates (D) in the mouse hippocampus. \#: 72 80 kDa tau isoform, *: $68 \mathrm{kDa}$ tau isoform, arrow head indicates $\sim 64 \mathrm{kDa}$ tau isoform, **: $\sim 110 \mathrm{kDa}$ high molecular weight tau isoform. (C) Representative confocal images of immunohistochemistry on the hippocampus of HSF1 +/- and WT at 13 months of age. co-immunostaining of p-Tau (Ser202/Thr205, red), pPERK (Thr980) (green), and DAPI (blue) in HSF1+/-. Arrow heads indicate colocalization of p-PERK and p-Tau (Ser202/Thr205) in the middle panels, enlarged images of top panels. Five repeated experiments performed. Scale bars : $5 \mu \mathrm{M}$. 
PERK, green) largely co- localized with of increased p-Tau (Ser202/Thr205, red) in the hippocampus of HSF1+/- at 13 months of age (Figure 4-3C). Of note, a dramatic upregulation of p-Tau (detected by both AT8 and AT100 antibodies) was strongly found in the hippocampus of 9 month-old HSF1+/- by western blot (Figure 4-3D, p-Tau (Ser202/Thr205), P < 0.01; p-Tau (Thr212/Ser214), P < 0.05, $\mathrm{n}=6$ ). These changes were not detectable in the whole brain lysates of 9 month-old HSF1 +/- (data not shown). Tau isolated from PHFs in human AD brain has been reported to contain $\sim 60-, \sim 64-$, and $~ 68$ $\mathrm{kDa}$ tau isoforms (Liu et al., 1993). In our study, the strongest immunoreactive band for phosphorylated tau protein in HSF1+/- was detected at $\sim 68 \mathrm{kDa}$ (Figure 4-3D). In the aged HSF1+/- hippocampus, there were two major p-Tau bands at $\sim 64-$ and $\sim 68-\mathrm{kDa}$ with a minor band at $\sim 72 \mathrm{kDa}$, similar to the pattern observed in human tauopathy brain (Spillantini et al., 1997) (Figure 4-3D). These are considered to be low molecular weight tau, ranging in 50-68 kDa on SDS-PAGE (Binder et al., 1985, Butler et al., 1986).

Sarkosyl insolubility assay is used to isolate tau paired helical filaments found in NFTs from AD brain (Greenberg et al., 1990). In the sarkosyl-insoluble fraction from the hippocampus of aged HSF1+/-, we found high molecular weight tau isoform, approximately $110 \mathrm{kDa}$ tau, in addition to a major band of $\sim 68 \mathrm{kDa}$ (Georgieff et al., 1991) (Figure 4-3D). Paired helical filaments from AD brain bind to Thioflavin S (SantaMaria et al., 2006). We did not observe any significant change in the expression levels of p-Tau and CHOP in the hippocampus of HSF1+/- at 2- and 6-months of age

(Figure 4-4). By double staining using thioflavin $\mathrm{S}$ and Tau antibody (Tau46), aggregates of hyperphosphorylated tau were found in the hippocampus of 13 month-old HSF1+/-, not in WT control (Figure 4-5). Diffuse plaques that showed strong positive signals for thioflavin S consisted of tau proteins (Figure 4-5). Thioflavin S staining also revealed globose-type NFTs and diffuse plaques in the cortical areas adjacent to the hippocampus (Figure 4-5).

Although tau protein is most abundant in axons, abnormal modifications of tau such as hyperphosphorylation can lead to redistribution of tau from neuronal processes to the soma where it likely forms toxic oligomers or aggregates (Tashiro et al., 1997). Hyperphosphorylation of tau has been identified to mislocalize tau to dendritic spines in neurons in AD models (Hoover et al., 2010). By subcellular fractionation and isolation of cytoplasm and synaptosome, both cytoplasmic- and synaptic- tau were found to increase in the hippocampus of 13 month-old HSF1+/- compared to WT control hippocampus (Figure 4-6A). We found increase in the average of the relative expression of $p$-Tau (Ser202/Thr205) in the cytoplasm and synaptosomal membrane of the hippocampus of 6 month-old HSF1+/- compared to that of 6 month-old WT (Figure 4-6A, cytoplasmic pTau (Ser202/Thr205), P < 0.05). Clearly, expression levels of p-Tau (Ser202/Thr205) were significantly upregulated in the both cytoplasm and synaptosomal membrane of the hippocampus of 14- and 25- month-old HSF1+/- compared to that of 14 month-old WT (Figure 4-6B). 


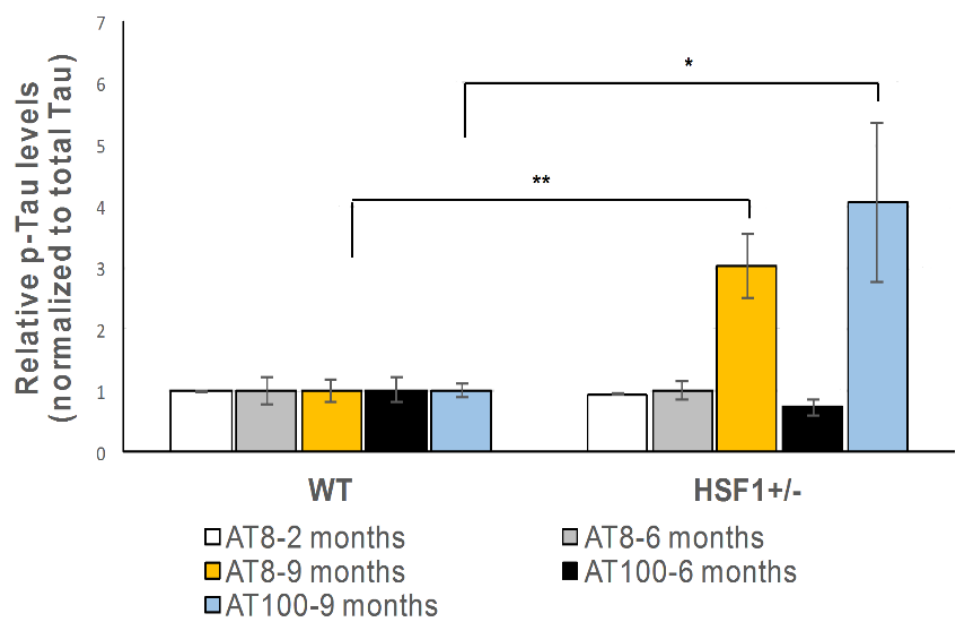

Figure 4-4. A Graph Shows Quantitative Measurement of the Relative Tau Phosphorylation Normalized to Total Tau (Tau46) in the Hippocampus of WT and HSF1+/- at 2, 6, and 9 Months of Age.

Two distinct p-Tau antibodies (AT8 (Ser202/Thr205) and AT100 (Thr212/Ser214)) used. (means $\pm \mathrm{SEM}, * P<0.05, * * P<0.01, \mathrm{n}=3$ ( 2 months), $\mathrm{n}=4$ (6 months) $\mathrm{n}=6$ (9 months)).

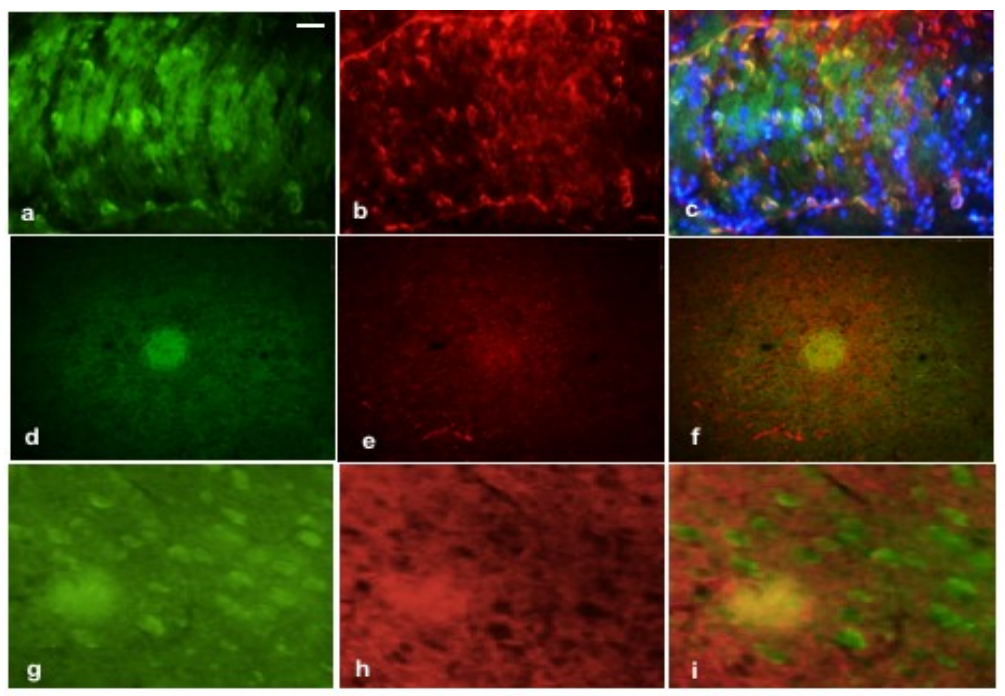

Figure 4-5. Double Staining with Thioflavin S (green, a) and Tau46 Antibody (red, b) in the Hippocampus of 13 Month-old HSF1 +/-.

Merged images of the hippocampus of HSF1+/- (c). Globose-type NFTs and diffuse plaques containing tau aggregates in the cortical areas adjacent to the hippocampus of HSF1+/- (d-i). Scale bar : $1 \mathrm{~mm}$. 


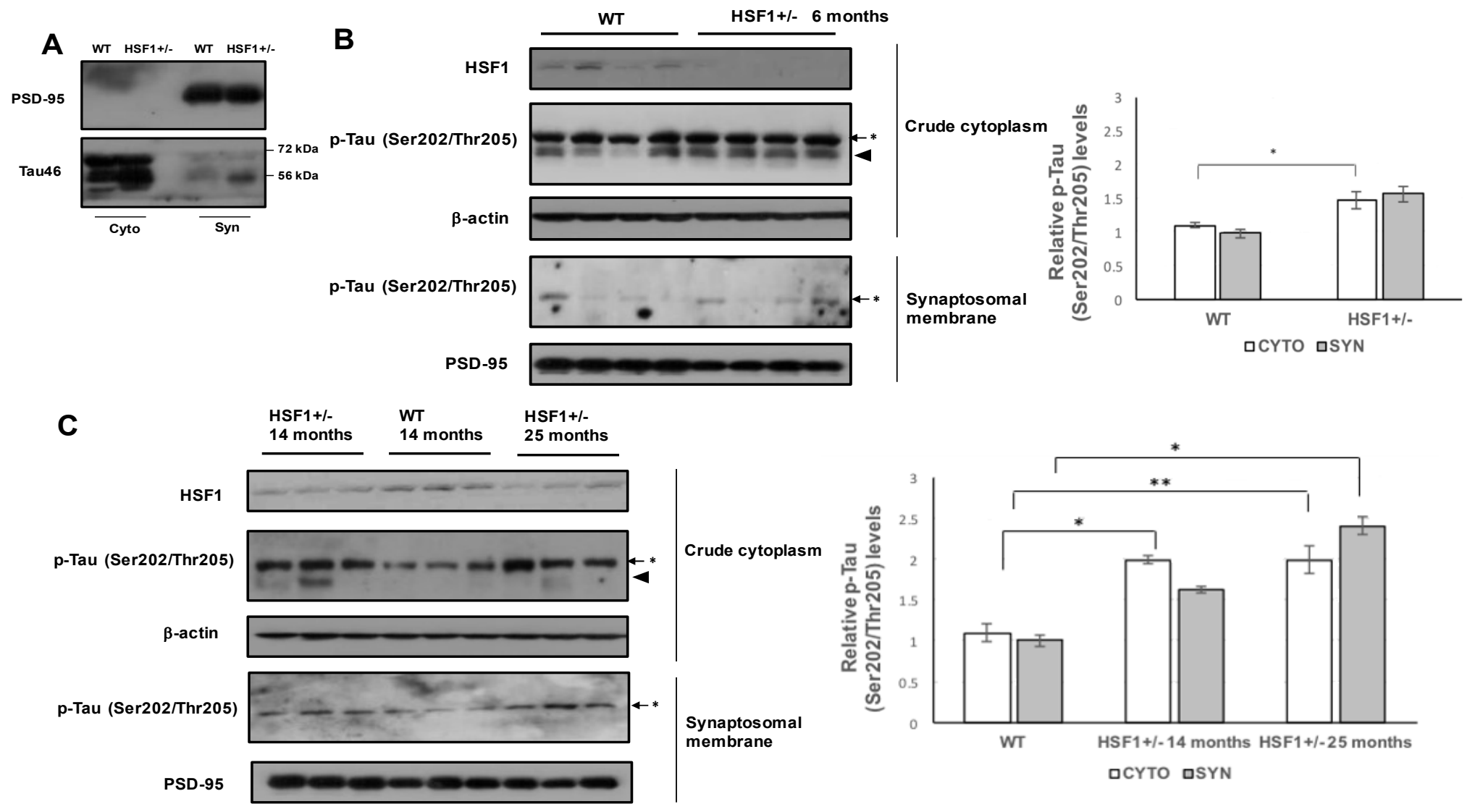

Figure 4-6. Abnormal Mislocalization of Hyperphosphorylated Tau in the Hippocampus of HSF1 Haploinsufficient Mouse. (A) PSD-95 highly enriched in the synaptosomal membrane fraction of 13 month-old mouse hippocampus. Tau 46 antibody was used to detect total tau protein in the crude cytoplasm and synaptosomal membrane fraction. (B and C) Increased expression of $\mathrm{p}-\mathrm{Tau}$ (Ser202/Thr205) in the cytoplasm and synaptosomal membrane fraction from the hippocampus of HSF1+/-. Graphs indicate quantification of p-Tau (Ser202/Thr205) protein levels normalized to $\square$-actin (cytoplasm) and PSD-95 (synaptosomal membrane) in the hippocampus of WT and HSF1+/- at different ages as indicated (means \pm SEM, $* \mathrm{P}<0.05, * * \mathrm{P}<0.01, \mathrm{n}=4(\mathrm{~B}), \mathrm{n}=3(\mathrm{C})$ ). 


\section{eIF2 $\alpha$-CHOP Activation Is Involved in Autophagy-Lysosomal HSF1 Protein Degradation during Chemically Induced ER Stress in Neurons}

We observed that loss of HSF1 protein was more severe in the brains of rTg4510 and Braak stages V/VI subjects where PERK was activated when compared to that of age-matched PS19 and stages III/IV subjects that did not show PERK activation, respectively (Figure 4-1, 4-2). Thus, we asked if UPR activation was involved in HSF1 protein loss in rat primary cultured neurons. Thapsigargin triggers ER stress by inhibiting Ca2+-transporting, ATPase mediated uptake of calcium ions into the sarcoplasmic reticulum. It is well documented that rapamycin, an inhibitor of mTOR (mammalian target of rapamycin), represses ER stress in various cell types (Kato et al., 2012). Rapamycin seemed to act as a reliable inhibitor of CHOP expression both in the presence and absence of thapsigargin in primary cortical neurons (Figure 4-7A, B, and $\mathbf{C}, \downarrow, \mathrm{P}<$ $0.001, \mathrm{n}=3-4)$. We demonstrated that thapsigargin triggered pro-apoptotic UPR activation that was attenuated by co-treatment of rapamycin (Figure 4-7A, B, and C). Of note, thapsigargin treatment led to $\sim 54 \%$ reduction in the HSF1 protein levels in primary neurons $(\mathrm{P}<0.05, \mathrm{n}=4)$, which was nearly blocked by rapamycin (Figure 4-7B, C). $\mathrm{CHOP}$ activation could be further enhanced by salubrinal treatment which inhibits activity of protein phosphatase 1 and prolongs eIF $2 \alpha$ phosphorylation. In contrast to rapamycin, salubrinal treatment further aggravated HSF1 loss from thapsigargin-induced

ER stress in primary neurons (Figure 4-7B, $\downarrow \downarrow$ ). Autophagy activity, as determined by LC3 II accumulation, was increased in thapsigargin-treated neurons (Figure 4-7B, $\downarrow$ ). Treatment of autophagy-lysosomal blocker, NH4Cl, inhibited thapsigargin-induced HSF1 loss, revealing HSF1 protein degradation through autophagy-lysosomal system (Figure 4-7B, a solid line indicated). Although direct HSF1 activator has not been identified, we previously reported that resveratrol could prevent HSF1 degradation from proteotoxic stress (Kim et al., 2016). In the current study, resveratrol attenuated thapsigargin-induced HSF1 protein degradation in primary neurons (Figure 4-7B, a dashed line indicated). Tunicamycin is another chemical UPR inducer that causes protein unfolding by blocking the glycoprotein synthesis pathway. Tunicamycin also reduced HSF1 protein expression in primary cultured cortical and hippocampal neurons (Figure 4-7D). We then sought in vivo evidence of thapsigargin-induced HSF1 degradation. Five month-old C57BL/6 mice were injected with thapsigargin (i.p. $2 \mathrm{mg} / \mathrm{kg}$ ) or PBS containing 10\% DMSO.

Remarkably, 6 hrs later, drastic HSF1 degradation, about 50\% reduction in HSF1 protein expression, was detected in the mouse brain injected with thapsigargin (Figure 4-7E, $\mathrm{P}<$ $0.01, \mathrm{n}=5)$.

\section{Overexpressed Pro-Aggregation Mutant Tau ${ }_{\text {RD }} \Delta$ K280 Leads to HSF1 Degradation Which Is Further Exacerbated by eIF2 $\alpha$-CHOP Activation}

Loss of HSF1 protein began to occur even in the absence of PERK activation in Braak stage III/IV and PS 19 mouse (Figure 4-1, 4-2). There is a possibility that proteotoxic stress from pathogenic tau affected HSF1 expression (Kim et al., 2016). To determine if overexpressed mutant tau caused HSF1 protein loss and eIF2 $\alpha-\mathrm{CHOP}$ 


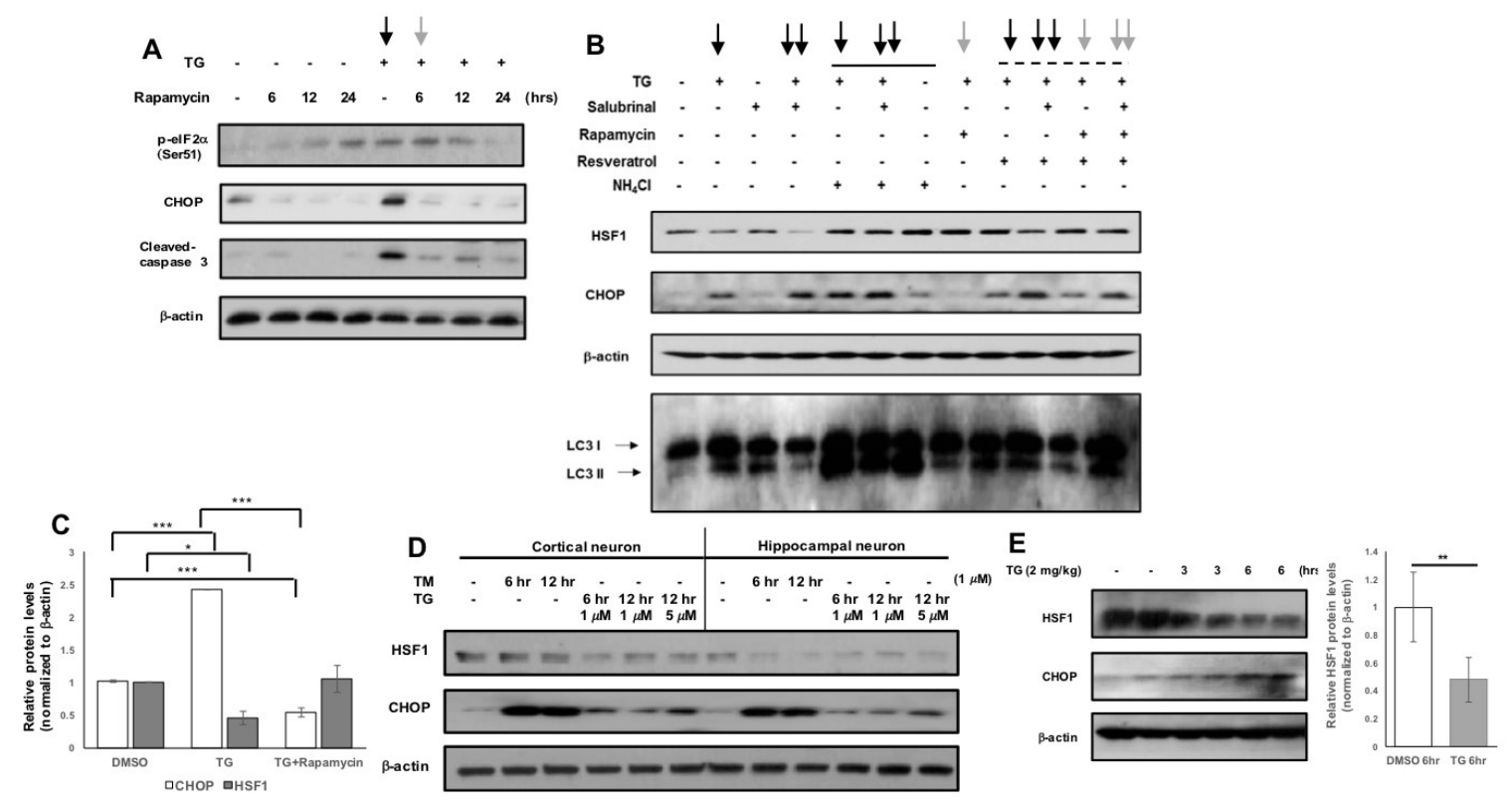

Figure 4-7. Autophagy-Lysosomal HSF1 Protein Degradation Mediated by eIF2 $\alpha$-CHOP Activation during Chemically Induced Acute ER Stress in Neurons.

(A) Reduced pro-apoptotic $\mathrm{CHOP}$ activation by rapamycin treatment during thapsigargin (TG)-induced ER stress. Primary cortical neurons were pre-treated with rapamycin $(0.4$ $\mu \mathrm{M}$, for indicated duration time) followed by TG incubation ( $1 \mu \mathrm{M}$, the last $6 \mathrm{hr}$ ). Cleaved-caspase 3 as apoptotic marker. (B) The combination treatments of ER stressinducer (TG, $1 \mu \mathrm{M}$ ), ER stress-inhibitor (rapamycin, $0.5 \mu \mathrm{M}$ ), p-eIF2 $\alpha$ activator (salubrinal, $20 \mu \mathrm{M})$, lysosomal blocker $\left(\mathrm{NH}_{4} \mathrm{Cl}, 5 \mathrm{mM}\right)$ and resveratrol $(5 \mu \mathrm{M})$ for $6 \mathrm{hr}$ in primary cortical neurons to study the relationship between HSF1 protein and eIF2 $\alpha$ CHOP activation. LC3 II increase by TG and $\mathrm{NH}_{4} \mathrm{Cl}$ (LC3 I and II (autophagy markers)). $\downarrow$ : thapsigargin, $\downarrow$ : co-treatment of thapsigargin and rapamycin, $\downarrow \downarrow$ : co-treatment of thapsigargin and salubrinal, A solid and a dashed line indicate the treatments of $\mathrm{NH}_{4} \mathrm{Cl}$ and resveratrol, respectively. (C) A graph represents quantification of relative protein levels of HSF1 and CHOP in the three distinct groups of primary cortical neurons (means $\pm \mathrm{SEM}, * P<0.05, * * * P<0.001, \mathrm{n}=3-4)$. TG $(1 \mu \mathrm{M}, 6 \mathrm{hr})$ and rapamycin $(0.5 \mu \mathrm{M}, 6 \mathrm{hr})$, (D) Rat primary cultured cortical and hippocampal neurons were treated with tunicamycin (TM) and TG at different doses $(1 \mu \mathrm{M}$ and $5 \mu \mathrm{M})$ and duration times $(6 \mathrm{hr}$ and $12 \mathrm{hr}$ ). (E) Cerebral cortex of 5 month-old B6 mice subjected to the intraperitoneal injection of thapsigargin (TG, $2 \mathrm{mg} / \mathrm{kg}$ ) were harvested at different time points indicated and subjected to western blot. A graph showing the change on the relative protein levels of HSF1 in the mouse cortex after TG treatment $(* * P<0.01$, comparing to control injected with PBS containing 10\% DMSO, means \pm SEM, $n=5$ ). 
activation in vitro, we transiently transfected N2a neuroblastoma cells with wild-type- or P301L- or $\Delta \mathrm{K} 280-\mathrm{Tau}_{\mathrm{RD}}$. Tau protein typically does not form amyloid fibrils in vitro because of its intrinsic hydrophilic feature. The lack of amyloidogenic propensity can be overcome by using the tau repeated domain $\left(\mathrm{Tau}_{\mathrm{RD}}\right)$, the most commonly used form of truncated tau. The four conserved sequence motifs in this domain are essential for tau aggregation. Both eIF2 $\alpha$-CHOP activation and decreased HSF1 protein expression were manifested in $\triangle \mathrm{K} 280 \mathrm{Tau}_{\mathrm{RD}}$ transfected N2a cells (Figure 4-8A). Thus, we generated $\mathrm{N} 2 \mathrm{a}$ cells stably overexpressing $\mathrm{Tau}_{\mathrm{RD}} \Delta \mathrm{K} 280\left(\mathrm{~N} 2 \mathrm{a}-\mathrm{Tau}_{\mathrm{RD}} \Delta \mathrm{K} 280\right)$ as a cellular model to study the relationship of CHOP activation and HSF1 loss in the following studies. In $\mathrm{N} 2 \mathrm{a}-\mathrm{Tau}_{\mathrm{RD}} \Delta \mathrm{K} 280$, about $40 \%$ of HSF1 protein was lost whereas 2.3 -fold increase in $\mathrm{CHOP}$ protein was found in $\mathrm{N} 2 \mathrm{a}-\mathrm{Tau}_{\mathrm{RD}} \Delta \mathrm{K} 280$, which was statistically significant (Figure 4-8B, HSF1, $\mathrm{P}<0.01$; CHOP, $\mathrm{P}<0.05, \mathrm{n}=4$ ). We observed a remarkable HSF1 increase after treatment with each of three agents to inhibit autophagy-lysosome pathway and MG132, a proteasomal blocker, suggesting that HSF1 protein was degraded by both UPS and autophagy-lysosome in N2a-Tau ${ }_{\mathrm{RD}} \Delta \mathrm{K} 280$ (Figure 4-8C).

Since reduced HSF1 expression caused CHOP activation in the mouse brain (Figure 4-3), we asked if HSF1 degradation was related with CHOP activation in N2a$\mathrm{Tau}_{\mathrm{RD}} \Delta \mathrm{K} 280$. Overexpressed HSF1 WT reduced about $60 \%$ of CHOP protein expression in N2a-TauRD $\Delta \mathrm{K} 280$ (Figure 4-9A, $P<0.001, \mathrm{n}=4$ ). Conversely, eIF2 $\alpha$ CHOP activation seemed to further promote HSF1 degradation in N2a-TauRD $\Delta \mathrm{K} 280$ (Figure 4-9B, $\mathrm{P}<0.01, \mathrm{n}=4$ ), as seen in primary neurons in Figure 4. Suppression of CHOP activation by rapamycin attenuated thapsigargin-induced autophagy-lysosomal HSF1 degradation in N2a-Tau ${ }_{\mathrm{RD}} \Delta \mathrm{K} 280$ (Figure 4-9C). During thapsigargin and tunicamycin treatments for various time periods, reduction of CHOP expression via siRNA upregulated HSF protein expression in N2a-Tau $\mathrm{RD} \Delta \mathrm{K} 280$ (Figure 4-9D), which was statistically significant (Figure 4-9E, $\mathrm{P}<0.05, \mathrm{n}=4$ ). However, in the absence of thapsigargin, CHOP was revealed not to be a primary component in HSF1 degradation present in N2a-Tau ${ }_{\mathrm{RD}} \Delta \mathrm{K} 280$, as confirmed by the lack of statistically significant HSF1 change following CHOP silencing (Figure 4-9E).

\section{Aberrant HSF1 Degradation and HSP70 a5 (BiP/GRP78) Attenuation Are Associated with Tau Accumulation and Toxicity in Tauopathy}

HSP70 a5 (BiP/GRP78), a major ER chaperone protein, acts as a negative regulator of UPR signaling (Bertolotti et al., 2000, Gorbatyuk et al., 2013). The promoter of HSP70a5 possesses DNA sequences called heat shock elements where HSF1 can bind for transcriptional activation (Mori et al., 1992). In contrast to CHOP elevation, HSP70 a5 (BiP/GRP78) expression normalized to $\beta$-actin was slightly reduced in the human AD brains when compared to non-AD brains (Figure 4-10A, non-significant). In addition, the steady state level of HSP70 a5 (BiP/GRP78) was 70\% less and 60\% less in N2a-TauRD $\Delta \mathrm{K} 280$ and $\mathrm{rTg}(\operatorname{tauP} 301 \mathrm{~L}) 4510$ than their control, respectively (Figure 4-10B).

Overexpressed HSF1 protein in $\mathrm{N} 2 \mathrm{a}-\mathrm{Tau}_{\mathrm{RD}} \Delta \mathrm{K} 280$ not only significantly enhanced HSP70 a5 (BiP/GRP78) expression but also remarkably reduced Tau $\mathrm{RD}_{\Delta \mathrm{K} 280}$ 


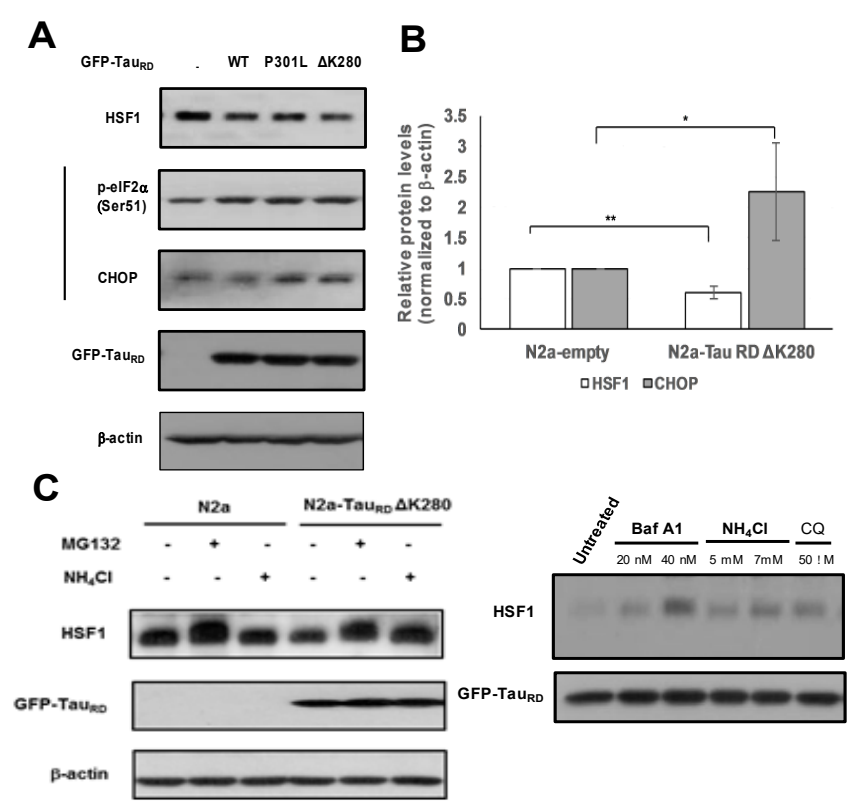

Figure 4-8. HSF1 Degradation and CHOP Activation in N2a-Tau ${ }_{R D} \Delta K 280$ Stable Cell Line.

(A) Western blot analysis on N2a neuroblastoma cells transiently overexpressing GFPtagged repeated domain (RD) tau constructs (TauRD WT, TauRD P301L, TauRD $\triangle$ K280) and empty vector (control). (B) A graph indicates relative protein levels of HSF1 and CHOP normalized to $\beta$-actin in $\mathrm{N} 2 \mathrm{a}$ and $\mathrm{N} 2 \mathrm{a}-\mathrm{Tau} \mathrm{RD} \Delta \mathrm{K} 280$ stable cell line (means \pm SEM, $* P<0.05, * * P<0.01$, comparing to control, $\mathrm{n}=4$ ). (C) HSF1 degradation through both autophagy-lysosome and proteasome in N2a-TauRD $\Delta K 280$. N2a-TauRD $\Delta K 280$ were treated with either autophagy-lysosomal (Baf $\mathrm{A} 1, \mathrm{NH}_{4} \mathrm{Cl}, \mathrm{CQ}$ ) or proteasomal blocker (MG 132, $20 \mu \mathrm{M}$ ) for $6 \mathrm{hr}$ at different concentrations indicated. Left: Control N2a treated with either MG132 or $\mathrm{NH}_{4} \mathrm{Cl}$. Right (short exposure of film): Baf A1: baflomycin A1, CQ: chloroquine. 
Figure 4-9. HSF1 Degradation Caused by Overexpressed TauRD $\Delta K 280$ and Further Facilitated by eIF2 $\alpha$-CHOP Activation.

(A) N2a-Tau ${ }_{\mathrm{RD}} \Delta \mathrm{K} 280$ were transfected with either HSF1 WT or HSF1 S303A (constitutively active form) or empty vector as control. Cleaved-caspase 3 as apoptotic marker. A right graph represents reduced relative $\mathrm{CHOP}$ protein expression levels normalized to $\beta$-actin in N2a-Tau $\mathrm{RD} \Delta \mathrm{K} 280$ overexpressing HSF1 WT (means $\pm \mathrm{SEM}$, $* * * P<0.001$, comparing to $\mathrm{N} 2 \mathrm{a}-\mathrm{Tau}_{\mathrm{RD}} \Delta \mathrm{K} 280$ transfected with empty vector, $\left.\mathrm{n}=4\right)$. (B) $\mathrm{N} 2 \mathrm{a}-\mathrm{Tau}_{\mathrm{RD}} \Delta \mathrm{K} 280$ were treated with either thapsigargin $(\mathrm{TG}, 1 \mu \mathrm{M})$ for $6 \mathrm{hr}$ or salubrinal $(20 \mu \mathrm{M})$ for $12 \mathrm{hr}$ or both of them. Their protein lysates were subjected to western blot (left). A graph on quantitative measurement of the relative HSF1 protein expression (right, means $\pm \mathrm{SEM},{ }^{*} P<0.05, \mathrm{n}=4$, N.S., non-significant). (C) Treatment of thapsigargin $(1 \mu \mathrm{M})$, rapamycin $(0.5 \mu \mathrm{M})$ and $\mathrm{NH}_{4} \mathrm{Cl}(5 \mathrm{mM})$ in $\mathrm{N} 2 \mathrm{a}-\mathrm{Tau}_{\mathrm{RD}} \Delta \mathrm{K} 280$. (D) The change on expression levels of HSF1 was assessed in N2a-TauRD $\Delta \mathrm{K} 280$ transfected with CHOP siRNA and later subjected to either thapsigargin or tunicamycin $(1 \mu \mathrm{M})$ treatment for different duration time as indicated. Quantitative measurement of relative HSF1 protein levels normalized to $\beta$-actin (bottom, arrow indicates dramatica increase in HSF1 protein by siCHOP expression.) (E) A graph indicates relative HSF1 protein levels in N2a-TauRD $\triangle \mathrm{K} 280$ transfected with CHOP siRNA in the presence or absence of thapsigargin (means $\pm \mathrm{SEM}, * P<0.05$, N.S., non-significant, $\mathrm{n}=3$ ). 


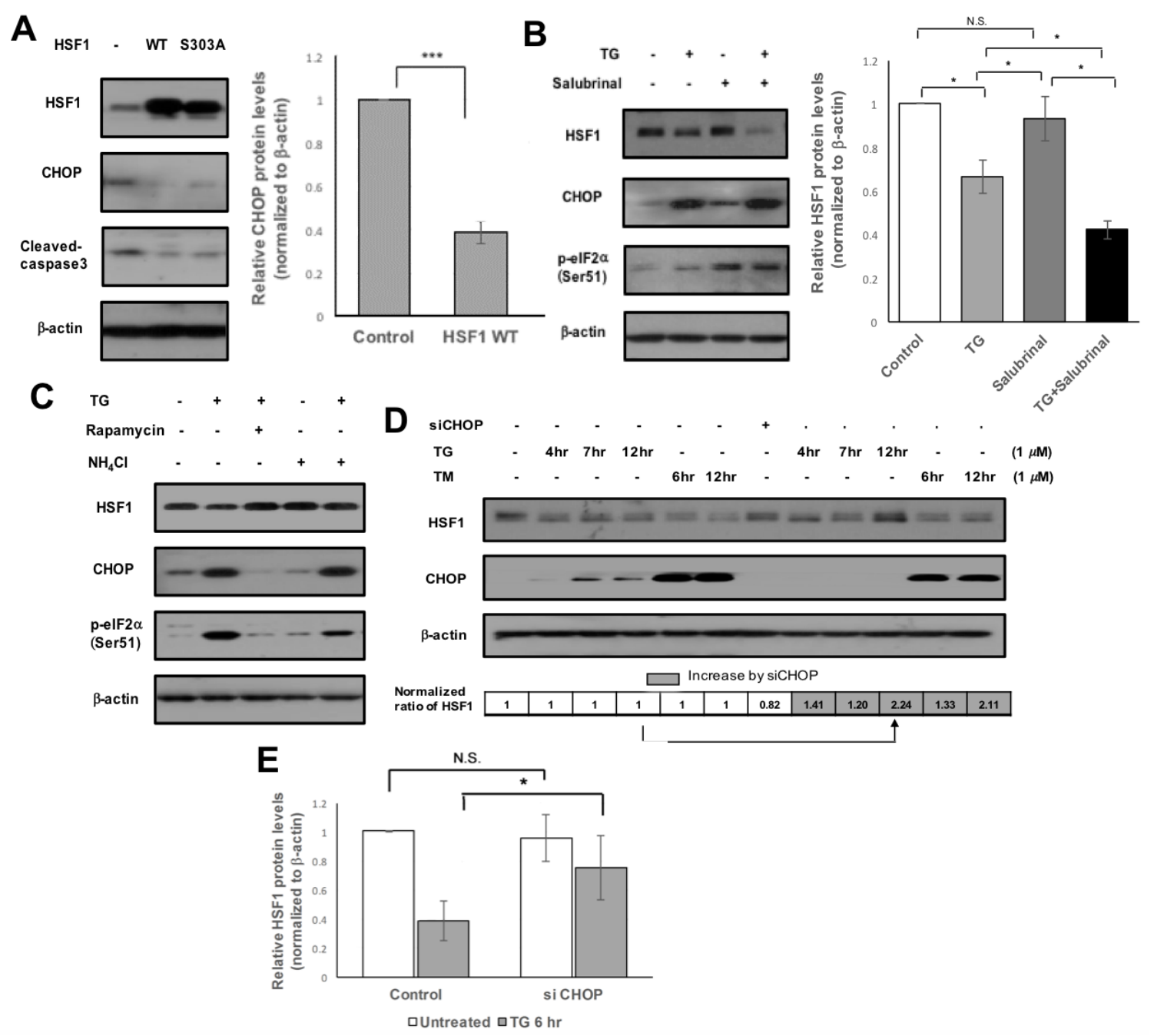



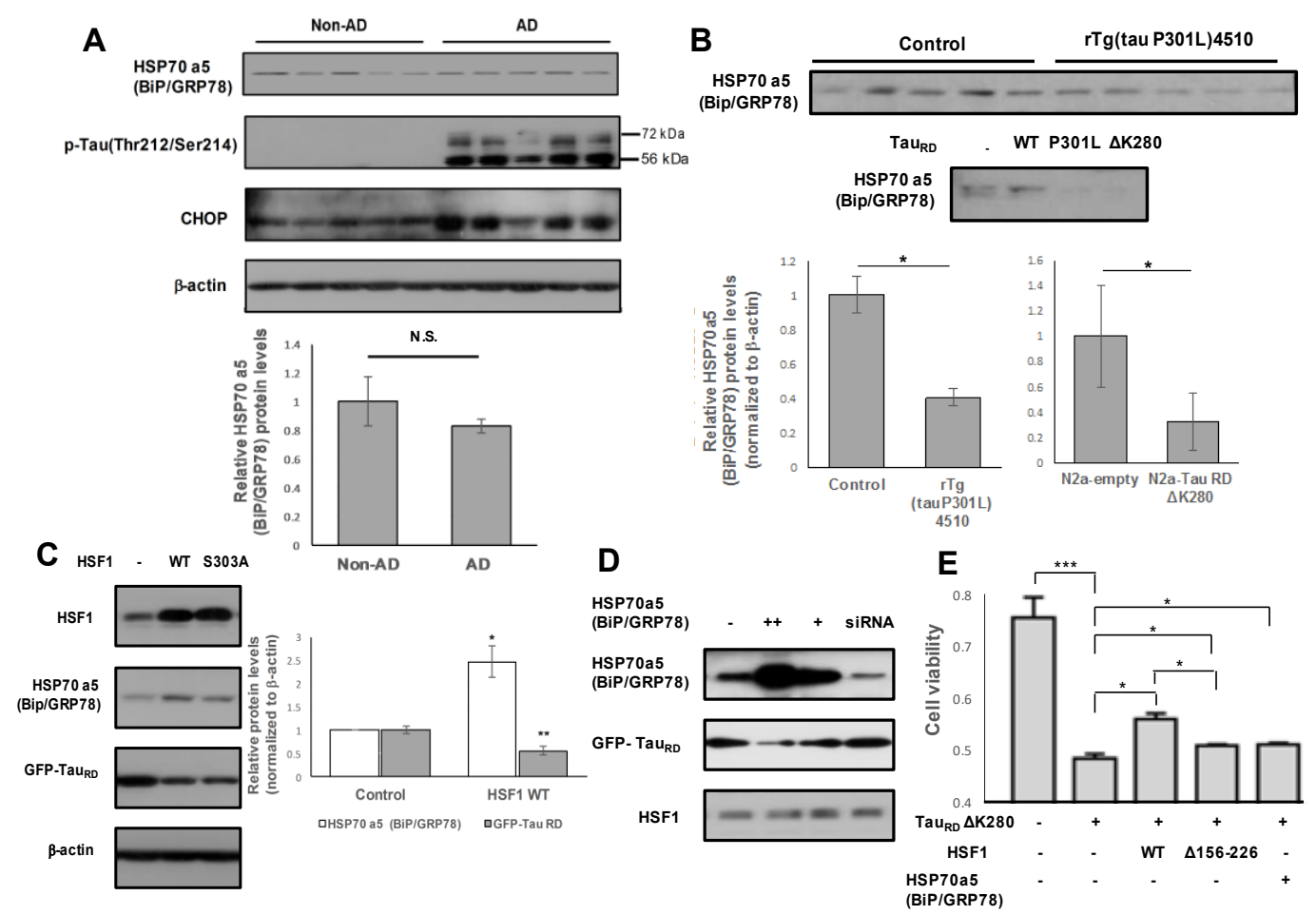

Figure 4-10. The Involvement of Aberrant HSF1 Degradation and HSP70 a5 (BiP/GRP78) Attenuation in Tau Accumulation and Toxicity in Tauopathy. (A and B) Reduced HSP70 a5 (BiP/GRP78) expression common in human AD brains (A) and cellular and mouse tauopathy models (B). Graphs show reduction in relative HSP70 a5 (BiP/GRP78) protein expression normalized to $\beta$-actin in N2a-Tau ${ }_{\mathrm{RD}} \Delta \mathrm{K} 280(\mathrm{n}=4$, * $P<0.05)$, human AD brains $(\mathrm{n}=5$, non-significant $)$ and $\operatorname{rTg}(\operatorname{tauP} 301 \mathrm{~L}) 4510(\mathrm{n}=5, * P$ $<0.05)$. Data represents means \pm SEM. $\beta$-actin for Fig. 6B was shown in Fig. 1B and 5A. (C and D) N2a-TauRD $\Delta \mathrm{K} 280$ were transfected with either HSF1 WT or HSF1 S303A (constitutively active form) (C) or HSP70 a5 (BiP/GRP78) or BiP siRNA (D). Control $\mathrm{N} 2 \mathrm{a}-\mathrm{Tau}_{\mathrm{RD}} \triangle \mathrm{K} 280$ transfected with empty vector. (C) A right graph on the relative HSP70 a5 (BiP/GRP78) and GFP-TauRD protein expression levels normalized to $\beta$-actin in N2a-Tau ${ }_{\mathrm{RD}}$ overexpressing HSF1 WT (means $\pm \mathrm{SEM},{ }^{*} P<0.05, \mathrm{HSP} 70$ a5 (Bip/GRP78); **P<0.01, GFP- Tau ${ }_{\mathrm{RD}}$, comparing to control, $\left.\mathrm{n}=4\right)$. (D) Reduced GFP$\mathrm{Tau}_{\mathrm{RD}}$ accumulation by HSP70 a5 (BiP/GRP78) overexpression in N2a-TauRD $\Delta \mathrm{K} 280$. (E) Cell viability in N2a-Tau ${ }_{\mathrm{RD}} \Delta \mathrm{K} 280$ overexpressing either HSF1 WT or HSF1 $\Delta 156-$ 226 , or HSP70 a5 (BiP/GRP78) and control was measured by MTT assay (means $\pm \mathrm{SEM}$, $* P<0.05, \mathrm{n}=5$ ) 
(Figure 4-10C, HSP70 a5 (BiP/GRP78), P < 0.05; GFP-Tau ${ }_{R D}, \mathrm{P}<0.01, \mathrm{n}=4$ ). Overexpressed HSP70 a5 (BiP/GRP78) reduced Tau RD $\Delta$ K280 accumulation without affecting HSF1 protein expression in N2a-Tau $\mathrm{RD}_{\mathrm{N}} \triangle \mathrm{K} 280$ (Figure 4-10D). These enhanced expressions of HSF1 and HSP70 a5 (BiP/GRP78) led to increased cell survival in N2a-Tau ${ }_{R D} \Delta$ K280 (Figure 4-10E). Overexpressed HSF1 mutant (i.e. HSF1 $156-226$ ) deficient in trimerization of a prerequisite step for transcriptional activation still demonstrated increased cell viability, however to a lesser degree than observed in HSF1 WT (Figure 4-10E). In primary neurons and N2a cells (Figure 4-11A, B), in response to tunicamycin treatment, protective UPR was activated that increased expression of HSP70 a5 (BiP/GRP78) (Figure 4-11A, B, $\mathrm{P}<0.05, \mathrm{n}=4$ ). In contrast, this protective stress

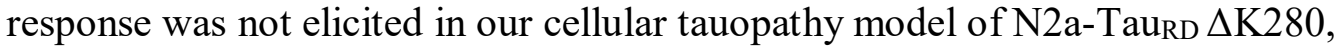
supporting attenuated HSP70 a5 (BiP/GRP78) expression in tauopathy (Figure 4-11A, B, $\mathrm{n}=4)$.

\section{Accumulation of Both $\alpha$-syn and Tau and Increased Activity of Tau Kinases Were Detected in the Brainstem of HSF1-haploinsufficient Mice}

In addition to hippocampus, tau hyperphosphorylation was significantly detected in the brainstem of HSF1+/- compared to age-matched control mice (Figure 4-12A). Hyperphosphorylation of $\alpha$-syn at Ser129 is found in Lewy body deposits and its neurotoxicitiy has been highly implicated in synucleinopathies (Sato et al., 2013). $\alpha$-syn and its phosphorylation at Ser129 both were found to be upregulated specifically in the brainstem of HSF1+/- at 13 months of age, whuch was absent in the hippocampus and cortex of HSF1+/- (Figure 4-12A). We also observed highly polyubiquitinated $\alpha$-syn in the brainstem of HSF1+/- (Figure 4-12B). Activation of tau kinases such as glycogen synthase kinase 3 beta (GSK3 $\beta$ ) and cyclin-dependent kinase 5 (CDK5) plays a key role in tau hyperphosphorylation in tauopatheis. Phosphorylation of GSK3 $\beta$ at a distinct site, Y216, is necessary for its activity. CDK5 is activated by binding to activator protein, $\mathrm{p} 35$, and its cleaved form of $\mathrm{p} 25$. Consistent with increased tau phosphorylation, expression of p35, p25, and p-GSK3 $\beta$ (Y216) was elevated in the brainstem of HSF1+/-

(Figure 4-12C). This increased activity of kinases was only detectable in the brainstem, not in the hippocampus and cortex of HSF1+/-. Protein phosphorylation is normally a reversible process regulated by phosphatases as well as kinases. In particular, protein phosphatase 2A (PP2A) is a principal tau-dephosphorylating enzyme in neurons. We did not observe any change on PP2A expression (Figure 4-12C).

\section{Summary}

Here we report studies in human brain and tau pathogenic mouse models (rTg4510 and PS19), identifying HSF1 degradation and UPR activation as precursors of aberrant tau pathogenesis. We demonstrate that chemical ER stress inducers caused autophagy-lysosomal HSF1 degradation, resulting in tau hyperphosphorylation in rat primary neurons. In addition, permanent HSF1 loss reversely causes chronic UPR activation, leading to aberrant tau phosphorylation and aggregation in the hippocampus of 

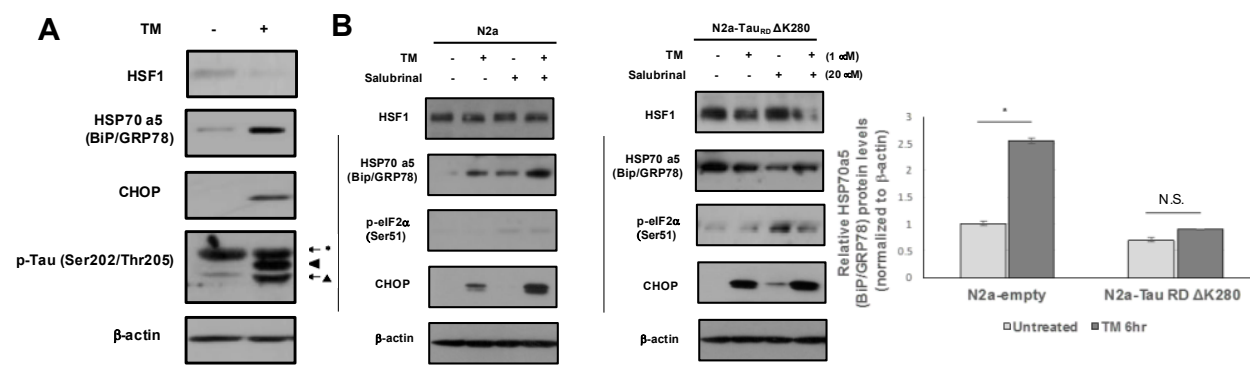

Figure 4-11. Suppressed Induction of HSP70 a5 (BiP/GRP78) upon Chemical ER Stress in N2a-TauRd $\Delta K 280$.

(A) Primary cortical neurons treated with tunicamycin (TM, $1 \mu \mathrm{M})$. (B) N2a-TauRD $\Delta \mathrm{K} 280$ and control N2a cells were treated with either tunicamycin $(\mathrm{TM}, 1 \mu \mathrm{M})$ for $6 \mathrm{hrs}$ or salubrinal $(20 \mu \mathrm{M})$ for $12 \mathrm{hrs}$ or both of them. Their protein lysates were subjected to western blot. A right graph on quantitative measurement of the relative HSP70 a5 $(\mathrm{BiP} / \mathrm{GRP} 78)$ protein expression in response to $\mathrm{TM}$ treatment in $\mathrm{N} 2 \mathrm{a}-\mathrm{Tau}_{\mathrm{RD}} \Delta \mathrm{K} 280$ and control N2a (means $\pm \mathrm{SEM},{ }^{*} P<0.05$, N.S., non-significant, $\mathrm{n}=4$ ).

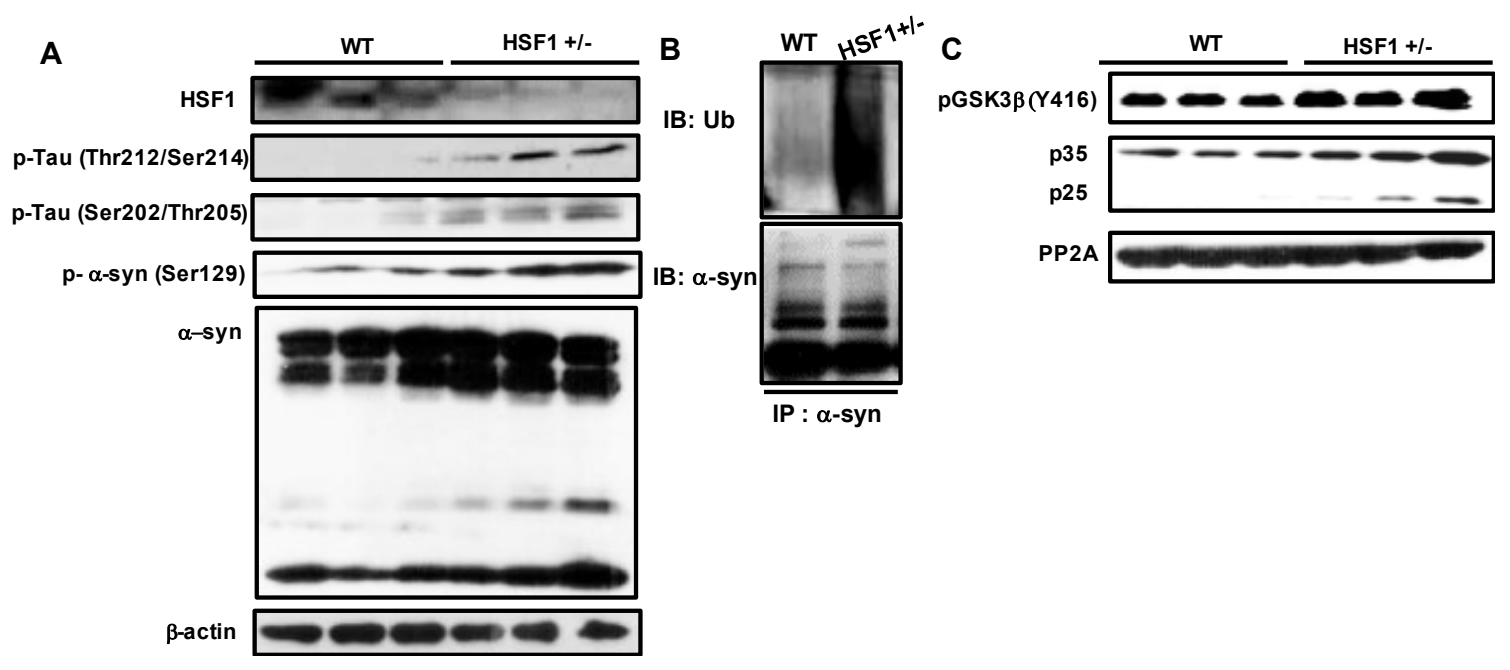

Figure 4-12. Accumulation of Both $\alpha$-syn and Tau and Increased Activity of Tau Kinases Were Detected in the Brainstem of HSF1+/-.

(A) Protein lysates from mouse brainstem were subjected to western blot to detect $\mathrm{p}$-Tau (Thr212/Ser214), p-Tau (Ser202/Thr205), p- $\alpha$-syn (Ser129), $\alpha$-syn, and $\beta$-actin. (B) Polyubiqutination of $\alpha$-syn in the brainstem of HSF1+/-. Co- immunoprecipitation of ubiquitin (Ub) with HSF1 using antibody against HSF1 was performed with protein extracts. (C) pGSK3 $\beta$ (Y416), p35/p25, and PP2A protein were detected by western blot. 
aged HSF1 heterozygous knock-out mice. The deleterious interplay of UPR activation and HSF1 loss is exacerbated in N2a cells stably overexpressing a pro-aggregation mutant TauRD $\Delta \mathrm{K} 280\left(\mathrm{~N} 2 \mathrm{a}-\mathrm{Tau}_{\mathrm{RD}} \Delta \mathrm{K} 280\right)$. We provide evidence of how these two stress response systems are intrinsically interwoven by showing that the gene encoding C/EBP-homologous protein (CHOP) activation in the UPR apoptotic pathway facilitates HSF1 degradation, which likely further contributes to prolonged UPR via ER chaperone HSP70 a5 (BiP/GRP78) suppression. Attenuating this vicious cycle by genetically or pharmacologically upregulating HSF1 relieves the tau toxicity in N2a- Tau RD $\Delta$ K280 by reducing CHOP and increasing HSP70 a5 (BiP/GRP78). Our work reveals how the bidirectional crosstalk between the two stress response systems promotes early tau pathology and identifies HSF1 being one likely key player in both systems. 


\section{CHAPTER 5. BEHAVIORAL CHARACTERIZATION OF HSF1 HAPLODEFICIENT MOUSE}

\section{Introduction}

In Chapters 3 and 4, we have studied the critical role of HSF 1 protein regulation in both synucleinopathy and tauopathy by biochemical analyses. We observed significant aging-associated AD-like neuropathological features, particularly, in the hippocampus of HSF1 haploinsufficient mouse (HSF1+/-). Expression of molecular chaperons is invovled in not only tau clearance but also rescuing behavioral deficits in tau transgenic mice (Ma et al., 2013). Thus, it is reasonable to hypothesize that reduced HSF1 contributes to neurodeneration and causes behavior deficits in mouse.

There is mounting evidence that the hippocampus plays an essential role in memory formation. The very first historical evidence came from Henry Molaison, whose unexpectedly disrupted hippocampus led to amnesia and failure of forming new episodic memories (Scoville et al., 1957). The hippocampus was first identified to regulate declarative long-term memory (Eichenbaum et al., 2004). Declarative memory indicates memory of facts and events that can be consciously recalled. Later, numerous studies have pointed out the hippocampus as a place responsible for working memory in the human brain (Axmacher et al., 2007, Poch et al., 2011). Working memory categorized as short-term memory enables temporary storage and managing the stored information that are necessary to execute complex tasks. In addition to the hippocampal function in memory, the hippocampus is involved in anxiety together with the amygdala and prefrontal cortex (Phillips and LeDoux, 1992, Sanders et al., 2003). The brainstem is a brain region where we found accumulation both $\alpha$-syn and tau protein in the aged HSF1+/- (Figure 4-12). The proper function of the the brainstem is necessary for making coordinated movements of the limbs and for maintaining posture. The brainstem is also involved in survival-related behaviors such as eating and drinking. The substantia nigra, a PD-affected area located in the midbrain, plays a key role in the regulation of movement, reward, and addiction. Taken together, it is intriguing to determine if there is any possible behavioral change including memory, anxiety, and locomoter activity in HSF1+/-.

Zhu et al., 2008 indicates that HSF1 deficient mice exhibited defective motivation and decreased exploratory behavior. Later, Uchida et al., 2011 also reports that HSF1 knock out mice showed reduced anxiety levels in a novelty-suppressed feeding test and an open field test. Using a forced swim test and a sucrose preference test, they demonstrated enhanced depression-like behavior in HSF1 homozygous knock-out mice. In the current chapter, we will extend our study to characterize the behaviors of aged HSF1+/- by performing a set of behavioral assays. 


\section{Results}

\section{Aged HSF1-haploinsufficient Mice Exhibit Increased Anxiety Levels in the Elevated Plus Maze, Not in the Open Field Test}

The previous studies from others reported statistically significant change on the anxiety levels only in HSF1 homozygous knock-out mice, not in heterozygous knock-out mice, compared to age-matched control mice, in the open field tests (Zhu et al., 2008, Uchida et al., 2011). Open-field test and elevated plus maze are commonly used to measure anxiety-like behaviors. In the current study, using open field test, we attempted to examine anxiety levels in HSF1-haploinsufficient mice (HSF1+/-) at two different ages, 13 months and 25 months, in comparison with 13 month-old HSF1+/+. In the open field test, all three groups of mice showed similar levels of total distance (Figure 5-1B). Total activity was found to be reduced in 25 month-old HSF1+/- compared to the other groups of mice (Figure 5-1A). In addition, time in center, which can be used as a measurement of anxiety levels, was increased in both different age groups of HSF $1+/$ - at almost similar levels (Figure 5-1C). However, all the differences observed were not statistically significant probably owing to large variation within groups.

In the elevated plus maze, we detected significant difference among groups in terms of time they spent in either open arm or closed arm or center zone (Figure 5-2B). Time spent in open arms was reduced in 13 month-old HSF1+/- when compared to HSF 1+/+, which was statistically significant (Figure 5-2B). 25 month-old HSF1+/- spent more time in closed arms and less time in open arms compared to 13 month-old HSF1+/-, (Figure 5-2B). The number of entries into open and closed arms, a parameter that represents locomoter activity of mouse, was increased in HSF1 +/- mice at two different ages compared to HSF1+/+ (Figure 5-2A).

\section{Aged HSF1-haploinsufficient Mice Exhibit Hippocampal-Dependent Learning and Memory Deficits}

Furthermore, we investigated short-term and long-term memory functions in the same group of HSF1+/- at two different ages, 13 months and 25 months, in comparison with 13 month-old HSF1+/+. Mice were tested in three independent behavioral assays, cross maze, fear contextual memory test, and morris water maze in order. First, the three groups of mice were subjected to the cross maze where two parameters, total entries and spontaneous alternation, were measured. The number of total entries as a reflection of activity was not different among groups (Figure 5-3A). Of note, working memory determined by spontaneous alteration ratio appeared to be significantly impaired in HSF $1+/$ - at both ages (Figure 5-3B). We observed no big difference between two groups of HSF1+/- at different ages (Figure 5-3).

Next, mice were subjected to the fear contextual retrieval test. Context-dependent memory enables mice to enhance the ability to recall specific information in the condition 
A

Total activity

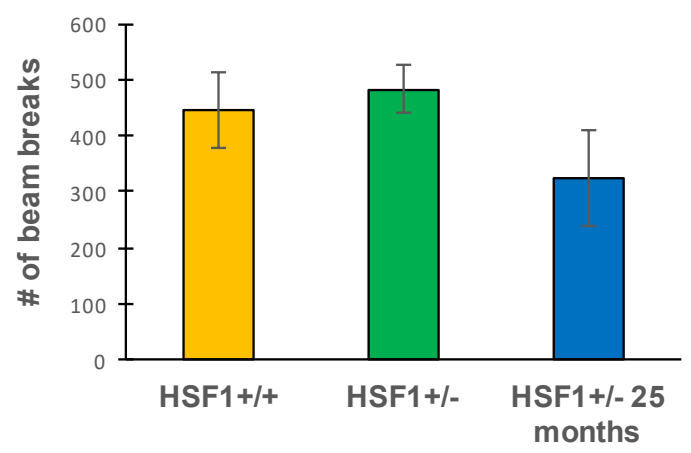

B

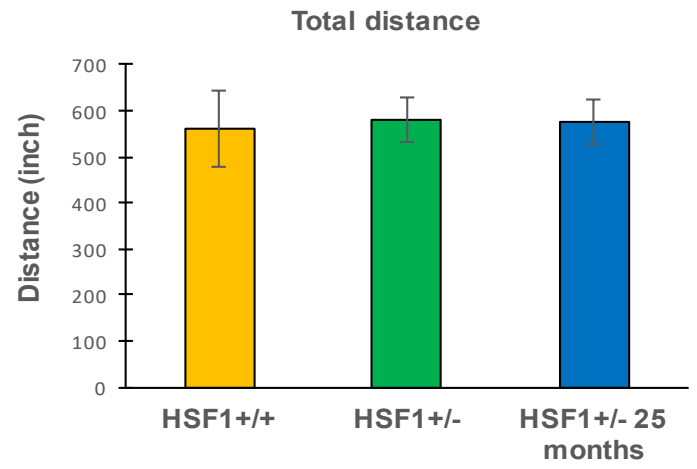

C Time in center

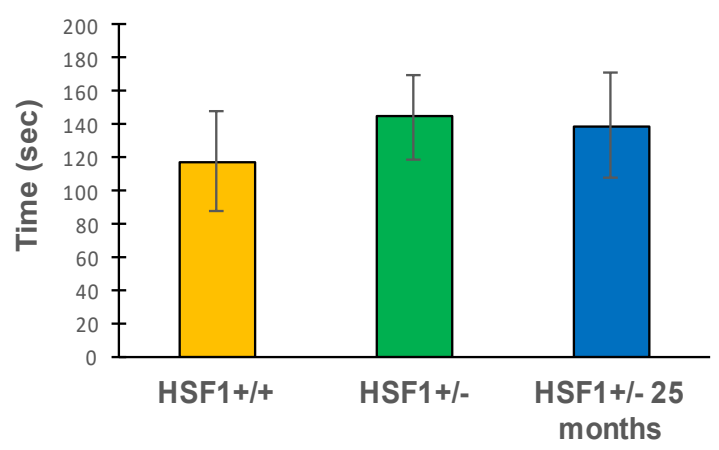

Figure 5-1. Locomoter Activity and Explorative Behavior in the Open Field Test. (A) Total number of beam breaks indicates locomoter activity. (B) Total distance travelled (unit : inch) represents locomoter activity. (C) Time spent in center was measured to assess explorative behavior. Means \pm SEM are represented in the graph. $\mathrm{n}=$ 10 (HSF1+/+, 13 months), $\mathrm{n}=17$ (HSF1+/-, 13 months), $\mathrm{n}=6$ (HSF1+/-, 25 months). 


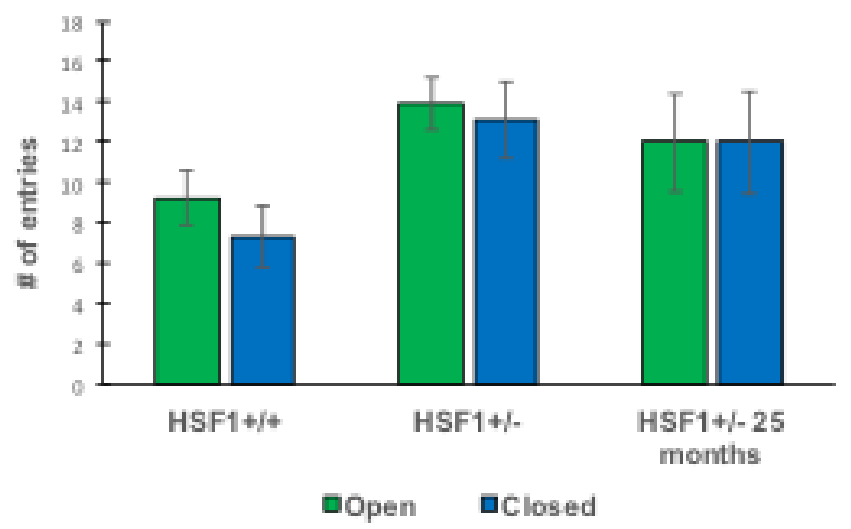

$\mathbf{B}$

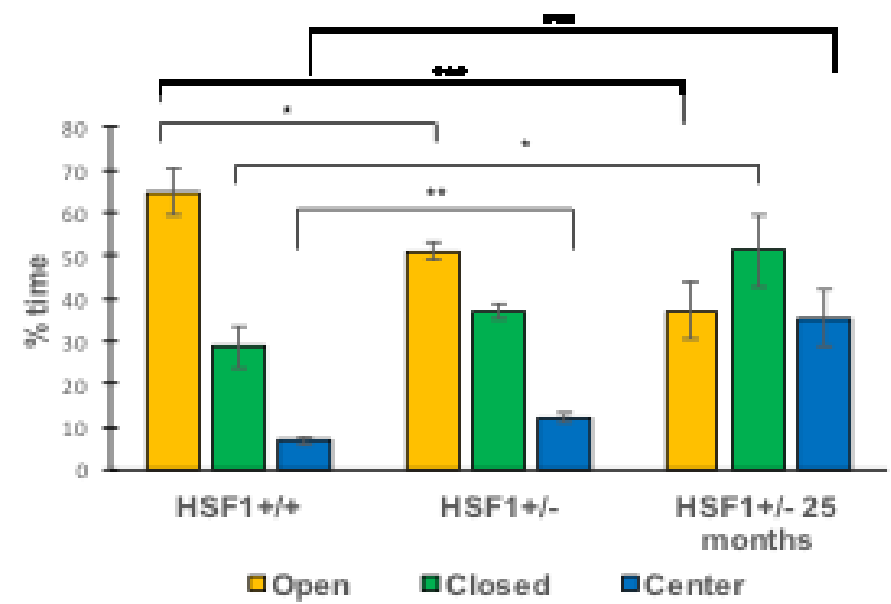

Figure 5-2. Increased Anxiety-related Behavior in HSF1-haploinsufficient Mice in the Elevated Plus Maze.

(A) Total number of entries into either open or closed arms. (B) Percentage of time spent in either open arms or closed arms or center zone was measured to assess anxiety-related behavior in three groups of mice. Means \pm SEM are represented in the graph. ${ }^{*} P<0.05$, ** $P<0.01, * * * P<0.001 \mathrm{n}=10$ (HSF1+/+, 13 months), $\mathrm{n}=17$ (HSF1+/-, 13 months), $\mathrm{n}=6$ (HSF1+/-, 25 months) 
A

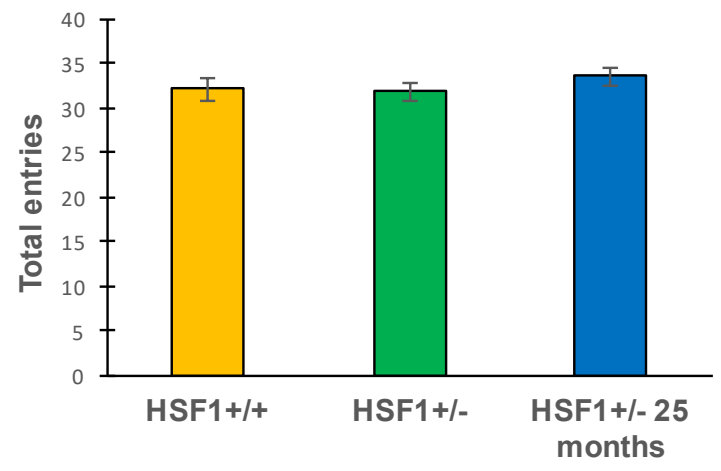

B

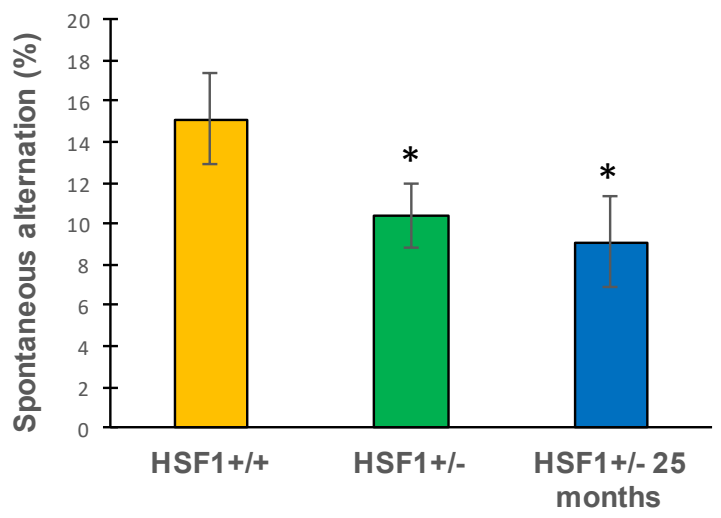

Figure 5-3. Impaired Working Memory in HSF1-haploinsufficient Mice in the Cross Maze.

(A) Total number of arm entries in HSF1+/+ (B) Ratios of spontaneous alternation versus total entry number were calculated. Means \pm SEM are represented in the graph. $* P<$ 0.05. $\mathrm{n}=10$ (HSF 1+/+, 13 months), $\mathrm{n}=17$ (HSF1+/-, 13 months), $\mathrm{n}=6$ (HSF1+/-, 25 months) 
that contexts exposed at encoding and retrieval are the same. During the first day training session, behaviors of HSF1 +/- and HSF1+/+ generated similar curves in the graph where freezing percentage across time was described (Figure 5-4A). A freezing percentage curve of 25 month-old HSF1+/- was shifted to downward in the graph (Figure 5-4A). As general, retrieval memory is measured by the freezing percentage on the second retrieval day. A freezing percentage was slightly increased in 13 month-old HSF1+/- compared to HSF1+/+, which was further elevated in 25 month-old HSF1+/- (Figure 5-4B). However, these changes were not statistically significant.

Finally, morris water maze test was employed to investigate learning and memory function in these mice. A graph that indicates total distance travelled across total 10 days hidden tests was shifted upward for HSF1+/- mice at 13 months of age compared to HSF1+/+ (Figure 5-5A). In other words, HSF1+/- tended to travel longer distance until they reached a platform. Learning and memory appeared likely to be defective in HSF1+/- at 25 months of age (Figure 5-5A). Reference memory represents learned performance based on representations that are found to be useful across trials. Reference memory was significantly reduced in HSF1+/- at 25 months of age (Figure 5-5B). HSF1+/- and HSF1+/+ showed comparable levels of swimming speed, supporting that decreased total distance in HSF1+/- was attributable to impairment in learning and memory as a result of HSF1 haplodeficiency (Figure 5-5C).

\section{Summary}

The data obtained from elevated plus maze and open field test in mouse has often been contradictory. It is desirable to carefully consider all the parameters that possibly confound the anxiety levels measured in these tests. Time in center, an indicator of anxiety-related behavior in the open field test, was slightly increased in HSF1+/compared to HSF1+/+ (Figure 5-1). Our results are consistent with the published data from Uchida et al., 2011. However, there is a possibility that increased locomoter activity of HSF1+/- might affects the interpretation of time in center in the test (Figure 5-1). In the elevated plus maze, although the number of total arm entries was upregulated in HSF1+/-, these mice exhibited significant decrease in the time spent in open arms. Time spent in open arms was further increased in HSF1 +/- at 25 months of age. It is argued that open filed test is suitable to measure locomoter and explorative behavirors whereas elevated plus maze is to evaluate anxiety-like behaviors. Thus, increased anxiety levels observed from elevated plus maze may be more reliable data than results from the open field test. Novelty suppressed feeding test can be great alternative to further study anxiety-related behaviors in HSF1+/-. This test is designed to assess stress-related anxiety levels by measuring the latency of mouse to eat a familiar food such as sucrose.

Our group previously showed that HSF1+/- spent less time with a novel object than wild-type control, suggesting impairment of episodic memory associated with the limbic system including hippocampus in HSF1+/- (Wang et al., 2016). In the current study, we garnered new evidence of impaired memory associated with damaged hippocampus in HSF1 +/- by several behavioral tests. Morris water maze and contextual 
A

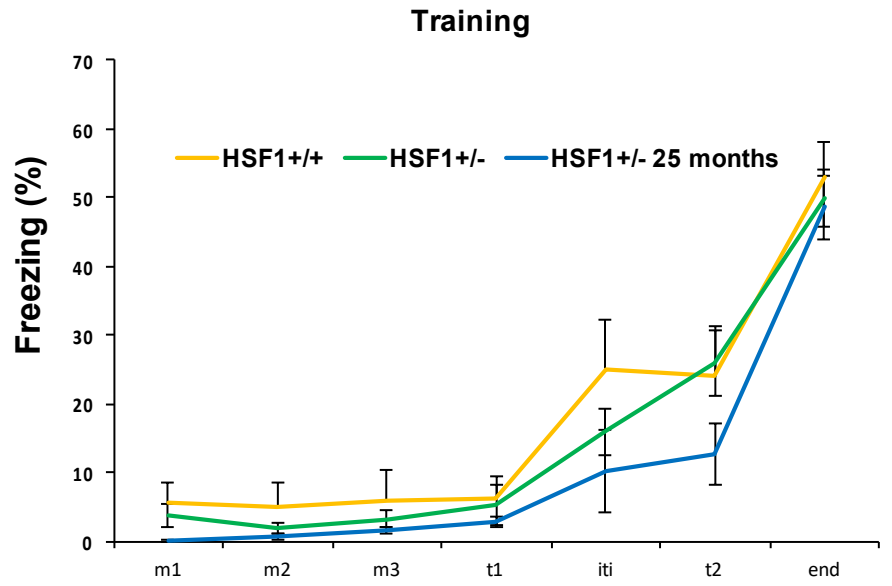

B

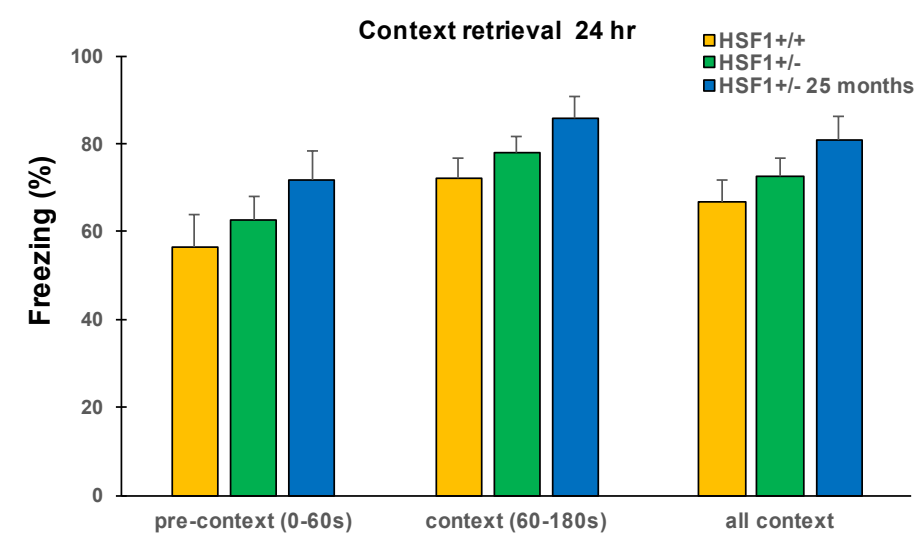

Figure 5-4. Fear Acquisition and Contextual Fear Memory in the Contextual Fear Conditioning Test.

(A) Freezing time (\%) during fear acquisition (training) in the contextual fear conditioning test. (B) Recorded contextual fear retention (context retrieval) $24 \mathrm{hr}$ after training. Data represents mean \pm SEM. $n=10$ (HSF1+/+, 13 months), $n=17$ (HSF1+/-, 13 months), $\mathrm{n}=6$ (HSF1+/-, 25 months) 
A

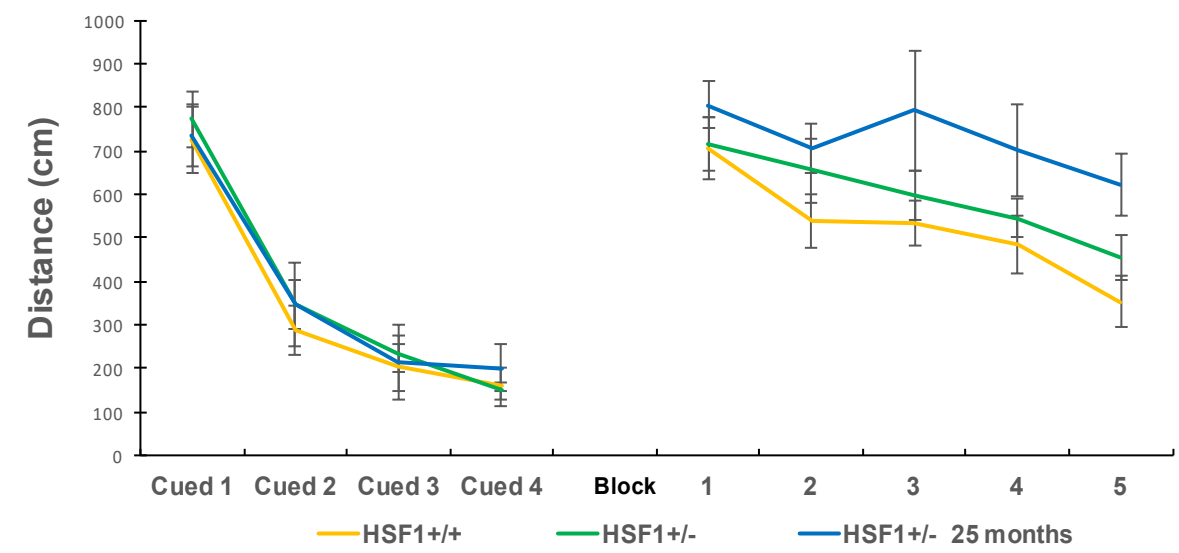

B

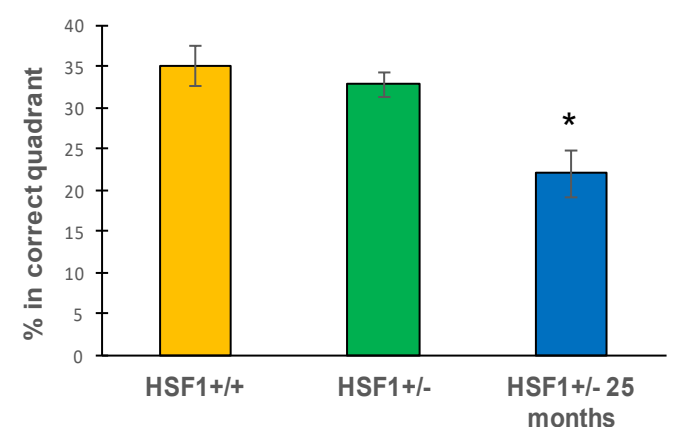

C

Figure 5-5. Defective Spatial Learning and Memory in HSF1-haploinsufficient Mice in the Water Maze.

(A) Distance swam was measured to examine spatial learning and memory in three different groups of mice. Histogram indicates average distance swam $(\mathrm{cm})$ in cued and hidden platform sessions. For analysis on hidden platform sessions, distance was averaged for every two days (total ten days hidden platform session, one block includes two days) (B) Probe trial test. After hidden platform sessions, percentage of time spent in the correct quadrant where platform was located was assessed to determine reference memory. (C) Swimming speed in the last two days of hidden session (block 5) was measured for three groups of mice. Data represents mean $\pm \mathrm{SEM} ; * P<0.05 . \mathrm{n}=10$ (HSF1+/+, 13 months), $\mathrm{n}=17$ (HSF1+/-, 13 months), $\mathrm{n}=6$ (HSF1+/-, 25 months). 
fear conditioning test are the most commonly used tasks to examine hippocampusdependent long-term memory. Contextual fear acquisition may involve both spatial and configural memory. Hippocampus is involved in contextual fear conditioning by affecting the input and output structure in fear conditioning (Phillips and LeDoux, 1992, Sanders et al., 2003). Amygldala also plays an important role in the formation and storage of fear memories. Disrupted amygdala caused failure in acquisiting and expressing fear-related memory (LeDoux et al., 1990). It should be noted that memory formation is not dependent on a single region, but rather determined by the interaction of neural circuitury. In the water maze which is a multi-trial task, it is hard to distinguish between deficits in learning versus memory. HSF1+/- seemed to have a difficulty in finding a platform compared to its control, which was further exacerbated in 25 monthold HSF1+/-. Because of a lack of statistical significance possibly due to large variation within each group in the water maze, repeated experiments with sufficient number of mice per each group need to be performed to consolidate our findings and make a conclusion. Defective working memory in HSF1+/- in the cross maze test further strengthens that HSF1 plays a crucial role in the formation and storage of memory related with functional hippocampus. 


\section{CHAPTER 6. DISCUSSIONS AND FURTHER DIRECTIONS}

\section{Aberrant HSF1 Degradation in Synucleinopathy}

Herein we have provided compelling and novel in vitro and in vivo evidence that proteotoxic stresses, as exemplified by $\alpha$-synucleinopathy, induce rapid and sustained HSF1 protein degradation mediated by the E3 ligase NEDD4 through UPS. We show here that both WT and A53T mutant $\alpha$-syn caused HSF1 protein degradation to varying degrees: while A53T $\alpha$-syn-induced HSF1 lost in both cytosolic and nuclear compartments, WT $\alpha$-syn over- expression appeared to only induce loss of nuclear HSF1. Whether nuclear HSF1 is more vulnerable to proteotoxic stress remains unclear.

The consequences of losing HSF1, a master regulator of stress response for cell survival, can be detrimental in many ways. For instance, impairment of HSF1-mediated stress response, reflected by failed induction of chaperone proteins to neuronal stresses can significantly impact the onset, development, and progression of stress-related neurodegenerative diseases. Additionally, given that HSF1 is an integrated part of proteosomal functions, even partial loss of HSF1, can further compromise proteasomal degradative machinery. This results in a build-up of misfolded substrate proteins that are labeled to be degraded by UPS (Pirkkala et al., 2000, Anckar et al., 2011). We recently discovered that HSF1 is the major transcription factor for activating not only hsp genes, but also a number of genes pertinent to synaptic functions, such as bdnf (Chen et al., 2014). Therefore, loss of HSF1 is expected to compromise synaptic plasticity and memory function. Given its various significant functions, loss of HSF1 may underlie a fundamental mechanism of neurodegeneration, featured by proteinopathies. It should be noted that HSF1 protein degradation appears to be a process selectively induced by proteotoxic stress, and not heat stress. In fact, heat stress induces HSF1 activation, and much of our understanding of HSF1 regulation has derived from the studies under heat shock conditions on various species: similarly to what an Hsp90 inhibitor does, heat shock in- duces an initial releasing step of HSF1 sequestration from Hsp90, followed by a series of phosphorylation, trimerization and nuclear translocation (Lindquist et al., 1986, Cotto et al., 1998, Zou et al., 1998, Shi et al., 1998, Morimoto et al., 1998). Multiple post-translational modifications of HSF1 have been studied in relation to its activity state, including acetylation, sumoylation and phosphorylation (Neef et al., 2011), and we can now add ubiquitination to that list.

Proteostasis has been most widely studied under heat shock conditions, and this HSR most notably concerns the HSF1 complex (Pirkkala et al., 2000). Proteostasis under proteotoxic stress, while less exten- sively studied, is believed to share much in common as HSR. However, it remains unclear how misfolded proteins are targeted for proteolysis under these conditions. Recently, Rsp5 and its mammalian homolog NEDD4 were discovered as the E3 ligases responsible for the increased ubiquitylation induced by heat stress. These ligases mainly target cytosolic misfolded proteins upon heat shock for proteasome degradation (Fang et al., 2014, Haitani et al., 2006). Interestingly, temperature-sensitive Rsp5 mutants revealed dysfunctional HSF1 transcriptional activity, 
as well as reduced protein expression of HSF1, conceivably due to some kind of posttranslational modification(s) (Haitani et al., 2008). In addition, physical interactions between Rsp5 and HSF1 were detected under this experimental condition. This work provided evidence of a modulatory role of Rsp5, as in its regulation of HSF1 during mRNA export from nucleus (Tardiff et al., 2013); however, it did not provide direct support for Rsp5 being the E3 ligase.

Herein, we provide compelling biochemical evidence for NEDD4 as the E3 ligase for HSF1 ubiquitination and the subsequence degradation via UPS. NEDD4 is the prototypic HECT - type E3 ligase for a large family that has been conserved from yeasts (Rsp5) to humans. NEDD4 was first identified by genetically screening developmentally down-regulated genes in the early embryonic, murine central nervous system (Yang et al., 2010, Donovan et al., 2013). Human NEDD4 exists in at least eight isoforms, resulting from alternative splicing that recognizes a proline-rich motif (PPXY or PY motif) not present in the HSF1 protein.

Strangely enough, NEDD4 was also reported to be the E3 ligase of $\alpha$-syn in Parkinsonism via the endosomal-lysosomal pathway (Tofaris et al., 2011). Defective Rsp5/NEDD4 pathways were linked to $\alpha$-synucleinopathy, which was supported by genetic screening in yeast (Tardiff et al., 2013, Chung et al., 2013). We consistently found that overexpression of WT NEDD4, but not the DN mutant form, resulted in a significant reduction of both ROS and $\alpha$-syn aggregation (Figure 3-6A, B). The seemingly contradictory roles of NEDD4 in the degradation processes of both toxic $\alpha$-syn and protective HSF1 raise possibility of involving different mechanisms. Although our current in vitro data suggest that HSF1 is primarily degraded via UPS, we cannot rule out possible involvement of other major lysosomal path- ways, particularly the chaperonemediated autophagy (CMA). In addition, different isoforms of NEDD4 may ubiquitinate $\alpha$-syn and HSF1, subjecting them to different subcellular compartments that likely involve different co-chaperone networks. Nevertheless, our study seeks to identify NEDD4 as an important E3 ligase, much like the C-terminus of Hsc70-interacting protein and Parkin in neurodegeneration.

Protein acetylation/deacetylation has been recently linked to protein regulation, especially via complex interplay with other forms of post-translational modifications (Caron et al., 2005). K208 and K298 acetylation by the acetyltransferase EP300 has been reported to regulate HSF1 protein stability under heat stress during reorgan- ization of nuclear proteosomal network (Raychaudhuri et al., 2014). Although it may be coincidental, acetylation has recently been identified as a mechanism regulating tau turnover (Cook et al., 2014), implying that complex interplay between different posttranslational modifications represents a common mechanism neurons use to fine-tune major classes of molecular processes. Our study distinguishes a strong correlation between an increased acetylation of HSF1 and its degradation, which is contrary to the relationship identi- fied between acetylation and the degradation of FTDP tau (Min et al, 2010). Deacetylation of Lys 80 residue by SIRT1 activation has been shown to activate the transcriptional activity of HSF1 via assessing its promoter occupancy rate

(Westerheide et al., 2009). We demonstrate that the same Lys 80 acetylation state directly 
correlates with the ubiquitination levels and the stability of HSF1. The underlying mechanism is unclear. Since we observed opposite levels of ubiquitination on the K80R but not K80Q mutants, ubiquitination of HSF1 unlikely occurs on the Lys80. However, it is plausible that somehow highly acetylated Lys80 (e.g. K80Q) alters protein conformation, making it more accessible for NEDD4-mediated ubiquitination.

In summary, we discovered complete loss of HSF1 protein under various conditions of proteinopathies, suggesting that aberrant HSF1 protein degradation may represent a common and important key molecular mechanism underlying neurodegeneration. While HSF1 is increasingly being recognized as an important therapeutic target (Neef et al., 2011), it should be approached with caution, and HSF1's protein degradation should be taken into consideration. Pharmacological development of a feasible HSF1 stabiliing agent is thus of high importance. It can perhaps be used in combination with an HSF1-activating agent to combat neurodegeneration. Our study has also demonstrated the potential sig- nificance of SIRT1-mediated deacetylation in HSF1 stability, adding to the growing body of evidence of sirtuins in the regula- tion of proteostasis (SampaloMarques et al., 2015). Fully delineating the underlying mechanism of SIRT1-mediated proteostasis will facilitate development of this promising strategy.

\section{HSF1 Degradation as a Mechanistic Link between UPR Activation and Tau Phosphorylation}

Here we provide both in vitro and in vivo evidence that strongly suggests an autopropagating interplay of UPR activation and HSF1 degradation being a common pathogenic feature in both human $\mathrm{AD}$ and tau transgenic mouse $\mathrm{AD}$ models (Figure 6-1). Characterizations of ER stress on early-stage AD / MCI patients have been neglected in many studies. The underlying mechanisms leading to chronically sustained ER stress in human $\mathrm{AD}$ brain are not entirely understood. This experiment looks to identify persistent and striking HSF1 degradation as an integral component in the chronic UPR activation pathway that ultimately causes tau hyperphosphorylation in early AD pathogenesis.

It should be noted that proteotoxic stress from tau aggregation could promote destabilization of HSF1 protein in several ways that are not clearly understood (Figure 4-1, 4-8). We implicate CHOP activation in the UPR pathway as one of key players that partially contributes to HSF1 degradation in tauopathy (Figure 4-7, 4-9). CHOP

activation can be regulated in the ER not only by PERK-eIF2 $\alpha$ but also by IRE1 or other unknown ER-independent mechanism (Kato et al., 2012). Consequently, reduced steady state level of HSF1 is likely to trigger permanent PERK-CHOP activation (Figure 4-3) that reversely facilitates HSF1 loss, leading to tau hyperphosphorylation in tauopathy (Figure 4-3, 4-9A). It can therefore be reasonably concluded that suppression of proapoptotic protein CHOP could be an efficient means of protecting HSF1 from ER stressrelated tauopathy. However, it was also revealed that in the young HSF1+/- mouse brain, HSF1 depletion alone was insufficient to cause either ER stress or tau phosphorylation (Figure 4-4), but another unidentified aging-related pathway with which HSF1 degradation associates with may actually cause disturbances in ER homeostasis. The 
A

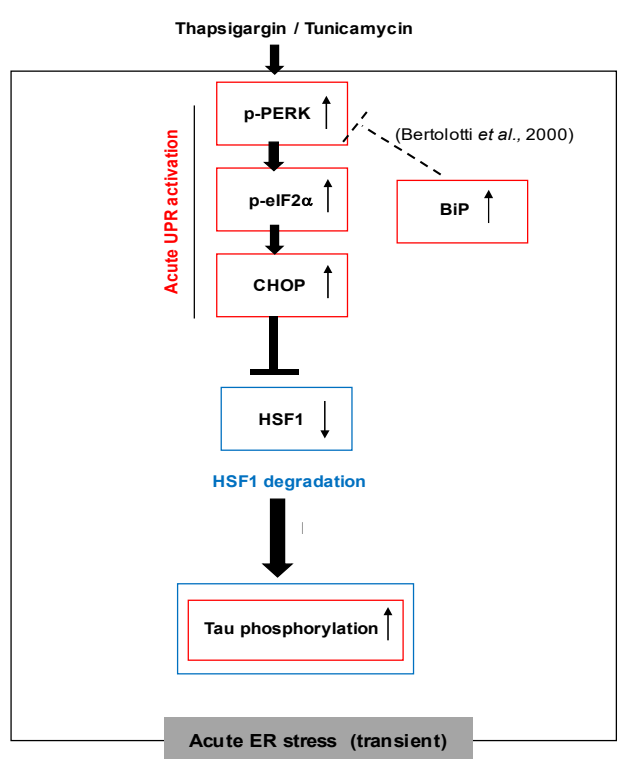

B

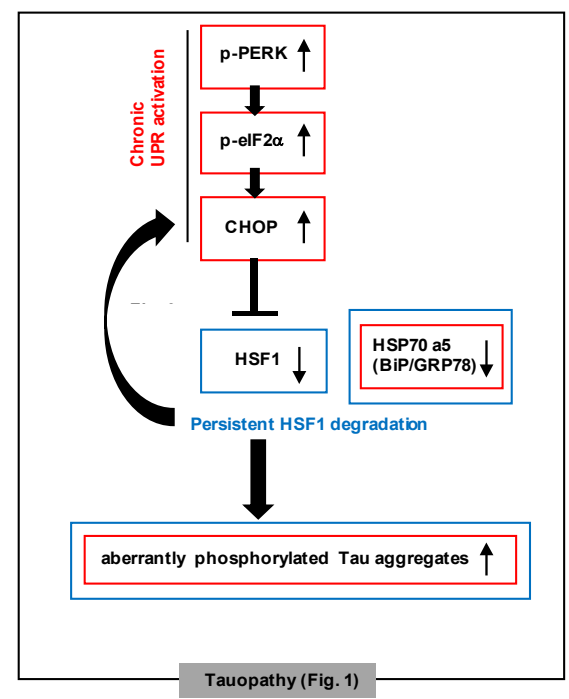

Figure 6-1. Hypothetic Model on a Vicious Cycle of UPR Activation and HSF1 Degradation in Tauopathy.

(A) Acute ER stress activates both CHOP and BiP proteins, which may 'transiently' lead to HSF1 degradation (Figure 4-4) and tau phosphorylation. Activated BiP contributes to cease UPR activation. (B) Chronic UPR activation (CHOP activation and BiP attenuation) in tauopathy. Persistent HSF1 loss seemed to cause chronic CHOP activation (Figure 4-2, 4-3), starting to form a vicious cycle of chronic CHOP activation and persistent HSF1 degradation. This eventually may lead to the formation of aberrantly phosphorylated tau aggregates in the aged hippocampus (Figure 4-2, 4-3). HSP70 a5 (BiP/GRP78) attenuation may contribute to tau accumulation and its toxicity in tauopathy (Figure 4-6). (Red, UPR-related proteins; Blue, HSF1-regulated proteins). 
relevant kinase(s) affecting tau phosphorylation in CHOP-HSF1 axis should also be determined in further studies. In addition to the role of autophagy-lysosomal activity on the HSF1 protein turnover, given highly poly-ubiquitinated HSF1 protein in N2a-TauRD $\triangle \mathrm{K} 280$, it is necessary to investigate the involvement of ubiquitin-proteasome system in tauopathy as we did in synucleinopathy (Kim et al., 2016). Tau toxicity can impair another essential response in ER called Endoplasmic-reticulum-associated protein degradation (ERAD) (Abisambra et al., 2013). Hrd1 is an ERAD-associated E3 ubiquitin protein ligase previously found to interact with tau (Abisambra et al., 2013). We observed a tremendous decrease in Hrd1 expression in rTg4510 (data not shown). The potential interactions between ERAD and HSF1 should be considered in future studies.

Considering HSF1 loss observed in Braak III/IV stages, it also leaves open the possibility of the involvement of amyloid pathology in HSF1 degradation. We found that the extent of HSF1 loss was more severe in tau transgenic mice (Tg4510) than in APP transgenic mice ( $\operatorname{Tg} 2576$ ) that produced amyloid beta plaques (Wang et al., 2016) (Figure 4-1). Tg2576 mice were reported not to show any signs of UPR activation by others (Lee et al., 2010). However, the more recent in vitro data suggests possible involvement of UPR activation in A $\beta$ toxicity (Resende et al., 2008). It is thus necessary to identify whether $A \beta$ can cause ER stress in vivo, and if so, whether or not this mechanism is tau-dependent.

Another corollary of HSF1 loss represented in the study is that it can be deterministic of the balance between pro-apoptotic (CHOP) and pro-survival responses (BiP) to ER stress. Inhibited HSP70 a5 (BiP/GRP78) protein expression is consistently observed in all our tauopathy models (N2a-Tau $\mathrm{RD}_{\mathrm{N}} \Delta \mathrm{K} 280, \mathrm{rTg}(\operatorname{taupP} 301 \mathrm{~L}) 4510$, and human AD, Figure 4-10). It may lead to an inability to attenuate UPR (Bertolotti et al., 2000, Gorbatyuk et al., 2013), contributing to tau aggregation and its toxicity in tauopathy. In vivo studies on Tau $\Delta \mathrm{K} 280$ transgenic mice indicated that tau toxicity was closely related to its ability to form aggregates (Mocanu et al., 2008). We could garner some evidence to speculate that the highly aggregated fibrillary form of tau is the major causative species to induce HSF1 degradation. Overexpressed full-length Tau WT, Tau P301L, and Tau R406W in N2a cells did not significantly alter HSF1 protein levels. Each tau repeated domain only (TauRD) and the mutant tau transgene lacking N-terminal insert (expressed in rTg4510) are all considered to aggregate faster than full length tau and the mutant tau transgene including one N-terminal insert (expressed in PS19), respectively (Barghorn et al., 2000, Binder et al., 2005, Gustke et al., 1994, VogelsbergRagaglia et al., 2000). The extent of HSF1 degradation may explain the discrepancies that exist in the presence of UPR activation in different tau transgenic mouse models (Shipton et al., 2011) (Figure 4-1).

The consequence of eIF2 $\alpha$ phosphorylation appears to be biphasic (Chadwick et al., 2012, Drexler et al., 2009). Though salubrinal is known to inhibit ER stress via protein synthesis attenuation (to prevent ER protein overload) (Boyce et al., 2005), elevated p-eIF2 $\alpha$ by salubrinal rather facilitated UPR-induced HSF1 degradation in N2a$\mathrm{Tau}_{\mathrm{RD}} \Delta \mathrm{K} 280$ of our study (Figure 4-9). Therefore, furthermore comprehensive consideration of both pathways and their functional outcomes in the cellular context is 
required. Our results are informative of the caution that should be taken in future designs of therapeutic approaches seeking to treat neurodegenerative diseases amenable to UPR inhibition.

\section{Synaptic Tau Pathology and HSF1 Dysregulation}

Interestingly, tau protein originally thought to be located in axon is now found to be present in dendritic spines as well, where it interacts with the Src kinase Fyn to regulate the NMDA receptor-mediated synaptic signaling (Ittner et al., 2010). Nonreceptor tyrosine kinase Fyn located in the postsynaptic density phosphorylates NMDA receptor subunit NR2B, leading to increase in the expression and activity of NMDA receptor. Tau protein mediates the translocation of Fyn to the exciatotory synpases (Ittner et al., 2010). In contrast, P301L mutant human tau was previously shown to reduce the number of glutamate receptors in spines, disrupting synaptic function (Hoover et al., 2010). Accumulation of tau in the somatodedritic and synaptic compartments in AD mouse models has been identified (Tashiro et al., 1997, Hoover et al., 2010). The presence of phosphorylated tau at synapses was not limited to human AD brain but also found in the adult human brain (Tai et al., 2012). In addition to its involvement in the microtubule stabilization, the normal physiological functions of synaptic tau in neurons should be further elucidated in detail. Tau deletion resulted in preventing hippocampaldependent behavior deficits in the AD mouse model overexpressing APP, revealing a central role of tau in cognitive impairment in AD (Roberson et al., 2007). Proper function of proteasome mediated protein turnover appears to be critical for synaptic transmission and plasticity on a time scale of minute (Speese et al., 2003). There is one study suggesting that synaptic tau aggregates were highly associated with impaired proteolysis through UPS (Tai et al., 2012). Rather than A $\beta$, tau protein well correlated with UPS impairment in synaptosomes in human AD brain. Attenuating tau-driven proteasome dysfunction prevented cognitive deficits in tau transgenic mouse model (Myegu et al., 2016). Therefore, impaired UPS function may be one potential mechanism by which synaptic tau causes cognitive deficits.

Neuronal hyperexcitability has been linked to both amyloid and tau pathology in AD mouse models (Busche et al., 2012). Neuronal activity has been implicated in the propagation of tau pathology (Wu et al., 2016, Pooler et al., 2013). Optogenetically stimulated rTg4510 mice overexpressing P301L human tau exacerbated somatic tau accumulation in the stimulated hippocampus (Wu et al., 2016). Exosomes represent small extracellular vesicles, which can be secreted and taken up by the recipient cells. Thus, it has emerged as a novel intercellular communication method to be used for the delivery of molecules (Asai et al., 2015, de Calignon et al., 2012). In attempts to understand transsynaptic transmission of tau, the concept of exosome-mediated tau transmission was first conceived by Saman et al., 2012. In the scenario, exosomes containing tau are released from axonal terminals where they can be taken up by relevant neurons in the neural circuit. Exosomes travelling to axon are finally released again at the presynaptic terminals, which contributes to the distribution of tau protein throughout the affected brain regions. In rat primary cortical neurons, it was demonstrated that neuronal activity 
induced release of tau-containing exosomes, which was dependent on synaptic connectivity (Pooler et al., 2013). Takeda et al., 2015 provided the first demonstration that a unique high-molecular weight tau is a specific form of tau involved in tau propagation throughout the brain. Accumulation of this high-molecular species was found in the cerebrospinal fluid of AD patients.

Importantly, we could garner substantial evidence that HSF1 plays a crucial role in the synaptic proteome in neurons. In the current study, we observed not only abnormally hyperphosphorylated tau protein present in synapses but also hippocampal memory deficits in HSF1+/-. It is pertinent to explore the possible role of UPS impairment in both tau accumulation and behavior deficits in HSF1+/-. HSF1 has been identified to be an important regulator of synaptic genes such as PSD-95 and BDNF. Of note, boosting HSF1 activity appears to provide synaptic protection in the AD mouse model by affecting coherent neuronal activity between the PFC and CA1 (Wang et al., 2016). It might be necessary to assess the electrophysiological features of neurons in HSF $1+/$ - in future studies.

The current study on the role of HSF1 dysregulation in neurodegeneration just began to highlight the importance of HSF1 protein stability to protect the brain from accumulation of misfolded proteins. This work reveals the pathological role of HSF1 degradation in both synucleinopathy and tauopathy. We observed that HSF1 haploinsufficiency in the brain could initiate neuropathological and behavioral changes that are associated with aging. It is necessary to identify the specific pathway targeted by aging that likely exacerbates defects as a result of HSF1 loss in the brain. AD has been featured as a supratentorinal disease and Braak staging of NFTs does not count for the braintem. However, interestingly, the case of braak stage 0 without any cortical tangles formation was shown to have NFTs in the dorsal raphe nucleus in the brainstem (Grinberg et al., 2009). This may suggest that NFTs burden could take place in the brainstem even before the supraentorhinal region. As each neurodegenerative disease targets particular subpopulation of neurons, we observed regionally specific pathologic findings in the brain of HSF1+/-. The findings on the brain stem of HSF1+/- where both pathological forms of $\alpha$-syn and tau were enormously accumulated should be further consolidated to make a firm conclusion. If it turns out to be true that brainstem is the foremost and utmost targeted area by HSF1 deficiency in terms of protein aggregates, it is intriguing to study the pathological synergistic effects of $\alpha$-syn and tau. It remains as a challenging task to uncover the direct and indirect cellular pathways that HSF1 loss activates or suppresses to increase intracellular aggregates in the brainstem 


\section{LIST OF REFERENCES}

Abisambra JF, Jinwal UK, Blair LJ, O'Leary JC, Li Q, Brady S et al. (2013) Tau accumulation activates the unfolded protein response by impairing endoplasmic reticulum-associated degradation. J Neurosci. 33:9498-9507

Ahn SG and Thiele DJ (2003) Redox regulation of mammalian heat shock factor 1 is essential for Hsp gene activation and protection from stress. Genes Dev. 17:516-528.

Akerfelt M, Morimoto RI, Sistonen L (2010) Heat shock factors: integrators of cell stress, development and lifespan. Nature Rev. Mol. Cell Biol. 11:545-555.

Alonso Adel C, Mederlyova A, Novak M, Grundke-Iqbal I, Iqbal K (2004) Promotion of hyperphosphorylation by frontotemporal dementia tau mutations. J Biol Chem 279: 34873-34881

Alzheimer's Association (2016) 2016 Alzheimer's disease facts and figures. Alzheimers Dement.12:459-509.

Anckar, J. and Sistonen, L. (2011) Regulation of HSF1 function in the heat stress response: implications in aging and disease. Annu. Rev. Biochem., 80, 1089-1115.

Andorfer C, Kress Y, Espinoza M, de Silva R, Tucker KL, Barde YA, Duff K, Davies P (2003) Hyperphosphorylation and aggregation of tau in mice expressing normal human tau isoforms. J Neurochem. 86:582-590

Anglade P, Vyas S, Javoy-Agid F, Herrero MT, Michel PP, Marquez J, Mouatt- Prigent A, Ruberg M, Hirsch EC, Agid Y (1997) Apoptosis and autophagy in nigral neurons of patients with Parkinson's disease. Histol Histopathol. 12:25-31.

Arima K, Hirai S, Sunohara N, Aoto K, Izumiyama Y, Uéda K, Ikeda K, Kawai M (1999) Cellular co-localization of phosphorylated tau- and NACP/alpha-synucleinepitopes in lewy bodies in sporadic Parkinson's disease and in dementia with Lewy bodies. Brain Res. 843:53-61.

Arrasate M, Mitra S, Schweitzer ES, Segal MR, Finkbeiner S (2004) Inclusion body formation reduces levels of mutant huntingtin and the risk of neuronal death. Nature. 431:805-10.

Arriagada PV, Growdon JH, Hedley-Whyte ET, Hyman BT (1992) Neurofibrillary tangles but not senile plaques parallel duration and severity of Alzheimer's disease. Neurology 42(3 Pt 1):631-639 
Asai H, Ikezu S, Tsunoda S, Medalla M, Luebke J, Haydar T, Wolozin B, Butovsky O, Kügler S, Ikezu T (2015) Depletion of microglia and inhibition of exosome synthesis halt tau propagation. Nat Neurosci. 18:1584-1593

Auluck PK, Chan HY, Trojanowski JQ, Lee VM, Bonini NM (2002) Chaperone suppression of alpha-synuclein toxicity in a Drosophila model for Parkinson's disease. Science. 295:865-868.

Axmacher N, Mormann F, Fernández G, Cohen MX, Elger CE, Fell J (2007) Sustained neural activity patterns during working memory in the human medial temporal lobe. J Neurosci. 27:7807-7816.

Barghorn S, Zheng-Fischhöfer Q, Ackmann M, Biernat J, von Bergen M, Mandelkow EM et al. (2000) Structure, microtubule interactions, and paired helical filament aggregation by tau mutants of frontotemporal dementias. Biochemistry 39:11714-11721

Barrachina M, Maes T, Buesa C, Ferrer I (2006) Lysosome-associated membrane protein 1 (LAMP-1) in Alzheimer's disease. Neuropathol Appl Neurobiol. 32:505-516.

Bartels T, Choi JG, Selkoe DJ (2011) $\alpha$-Synuclein occurs physiologically as a helically folded tetramer that resists aggregation. Nature. 477:107-110

Bence NF, Sampat RM, Kopito RR. Impairment of the ubiquitin-proteasome system by protein aggregation. Science. 2001; 292:1552-1555

Bennett EJ, Bence NF, Jayakumar R, Kopito RR (2005) Global impairment of the ubiquitin-proteasome system by nuclear or cytoplasmic protein aggregates precedes inclusion body formation. Mol Cell. 17:351-365.

Ben-Zvi A, Miller EA, Morimoto RI (2009) Collapse of proteostasis represents an early molecular event in Caenorhabditis elegans aging. Proc Natl Acad Sci U S A. 106:1491414919.

Bertolotti A, Zhang Y, Hendershot LM, Harding HP, Ron D (2000) Dynamic interaction of BiP and ER stress transducers in the unfolded-protein response. Nat Cell Biol. 2:326332

Binder L, Frunkfurter A, Rebhum L (1985) The distribution of tau in the mammalian nervous system. J. Cell Biol.101: 1371-1378

Binder LI, Guillozet-Bongaarts AL, Garcia-Sierra F, Berry RW (2005) Tau, tangles, and Alzheimer's disease. Biochim Biophys Acta 1739:216-223

Björkdahl C, Sjögren MJ, Zhou X, Concha H, Avila J, Winblad B, Pei JJ (2008) Small heat shock proteins Hsp27 or $\alpha \mathrm{B}$-crystallin and the protein components of neurofibrillary tangles: Tau and neurofilaments. J Neurosci Res. 86: 1343-1352 
Boyce M, Bryant KF, Jousse C, Long K, Harding HP, Scheuner D et al. (2005) A selective inhibitor of eIF2alpha dephosphorylation protects cells from ER stress. Science 307:935-939

Braak H, Braak E (1991) Neuropathological stageing of Alzheimer-related changes. Acta Neuropathol 82:239-259.

Braak H, Del Tredici K, Bratzke H, Hamm-Clement J, Sandmann-Keil D, Rüb U (2002) Staging of the intracerebral inclusion body pathology associated with idiopathic Parkinson's disease (preclinical and clinical stages). J Neurol. 249 Suppl 3:III/1-5.

Broquet AH, Thomas G, Masliah J, Trugnan G, Bachelet M (2003) Expression of the molecular chaperone Hsp70 in detergent-resistant microdomains correlates with its membrane delivery and release. J Biol Chem. 278:21601-21606

Brown SA, Weirich CS, NewtonEM,Kingston RE (1998) Transcriptional activation domains stimulate initiation and elongation at different times and via different residues. EMBO J. 17:3146-3154

Burke RE, Dauer WT, Vonsattel JP (2008) A critical evaluation of the Braak staging scheme for Parkinson's disease. Ann Neurol. 64:485-491

Busche MA, Chen X, Henning HA, Reichwald J, Staufenbiel M, Sakmann B, Konnerth A (2012) Critical role of soluble amyloid- $\beta$ for early hippocampal hyperactivity in a mouse model of Alzheimer's disease. Proc Natl Acad Sci U S A. 109:8740-8745

Butler M and Shelanski ML (1986) Microheterogeneity of microtubule-associated tau proteins is due to differences in phosphorylation. J. Neurochem. 47: 1517-1522

Cabin DE, Shimazu K, Murphy D, Cole NB, Gottschalk W, McIlwain KL, Orrison B, Chen A, Ellis CE, Paylor R, et al. (2002) Synaptic vesicle depletion correlates with attenuated synaptic responses to prolonged repetitive stimulation in mice lacking alphasynuclein. J Neurosci. 22:8797-8807.

Chadwick W, Mitchell N, Martin B, Maudsley S (2012) Therapeutic targeting of the endoplasmic reticulum in Alzheimer's disease. Curr Alzheimer Res 9:110-119

Chen B., Retzlaff M., Roos T. and Frydman J. (2011) Cellular strategies of protein quality control. Cold Spring Harb. Perspect. Biol., 3, a004374

Chen L, Feany MB (2005) Alpha-synuclein phosphorylation controls neurotoxicity and inclusion formation in a Drosophila model of Parkinson disease. Nat Neurosci.8:657663. 
Chen L, Thiruchelvam MJ, Madura K, Richfield EK (2006) Proteasome dysfunction in aged human alpha-synuclein transgenic mice. Neurobiol Dis. 23:120-126

Chen Y, Wang B, Liu D, Li JJ, Xue Y, Sakata K, Zhu LQ, Heldt SA, Xu H, Liao FF (2014) Hsp90 chaperone in- hibitor 17-AAG attenuates A $\beta$-induced synaptic toxicity and memory impairment. J. Neurosci., 34, 2464-2470.

Cleveland DW, Hwo SY, Kirschner MW (1977) Physical and chemical properties of purified tau factor and the role of tau in microtubule assembly. J Mol Biol 116: 227-247.

Cook C, Stankowski, JN, Carlomagno Y, Stetler C, Petrucelli L (2014) Acetylation: a new key to unlock tau's role in neurodegeneration. Alzheimers Res. Ther., 6, 29.

Cooper AA, Gitler AD, Cashikar A, Haynes CM, Hill KJ, Bhullar B, Liu K, Xu K, Strathearn KE, Liu F, et al. (2006) Alpha-synuclein blocks ER-Golgi traffic and Rab1 rescues neuron loss in Parkinson's models. Science. 313 :324-328.

Cotto J., Fox S. and Morimoto RI (1998) HSF1 granules: a novel stress-induced nuclear compartment of human cells. J. Cell. Sci., 110, 2925-2934.

Cuervo AM, Stefanis L, Fredenburg R, Lansbury PT, Sulzer D (2004)

Impaired degradation of mutant alpha-synuclein by chaperone-mediated autophagy. Science. 305:1292-1295.

Dai C, Whitesell L, Rogers AB, Lindquist S (2007) Heat shock factor 1 is a powerful multifaceted modifier of carcinogenesis. Cell. 130:1005-1018.

Dai Q, Zhang C, Wu Y, McDonough H, Whaley RA, Godfrey V, Li HH, Madamanchi N, Xu W, Neckers L, Cyr D, Patterson C (2003) CHIP activates HSF1 and confers protection against apoptosis and cellular stress. EMBO J. 22:5446-5458.

Davie CA (2008) A review of Parkinson's disease. Br Med Bull. 86:109-127.

Davies SE, Hallett PJ, Moens T, Smith G, Mangano E, Kim HT, Goldberg AL, Liu JL, Isacson O, Tofaris GK (2014) Enhanced ubiquitin-dependent degradation by Nedd4 protects against $\alpha$-synuclein accumulation and toxicity in animal models of Parkinson's disease. Neurobiol Dis. 64:79-87.

de Calignon A, Polydoro M, Suárez-Calvet M, William C, Adamowicz DH, Kopeikina $\mathrm{KJ}$ et al. (2012) Propagation of tau pathology in a model of early Alzheimer's disease. Neuron. 73: 685-697.

De Maio A (1999) Heat shock proteins: facts, thoughts, and dreams. Shock. 11:1-12. 
Desai S, Liu Z, Yao J, Patel N, Chen J, Wu Y, Ahn EE, Fodstad O, Tan M (2013)

Heat shock factor 1 (HSF1) controls chemoresistance and autophagy through transcriptional regulation of autophagy-related protein 7 (ATG7). J Biol Chem 288:91659176

Dettmer U, Newman AJ, Soldner F, Luth ES, Kim NC, von Saucken VE, Sanderson JB, Jaenisch R, Bartels T, Selkoe D (2015) Parkinson-causing $\alpha$-synuclein missense mutations shift native tetramers to monomers as a mechanism for disease initiation. Nat Commun. 6:7314.

Dickey CA, Eriksen J, Kamal A, Burrows F, Kasibhatla S, Eckman CB, Hutton M, Petrucelli L (2005). Development of a high throughput drug screening assay for the detection of changes in tau levels - proof of concept with HSP90 inhibitors. Curr Alzheimer Res. 2:231-238.

Dickey CA, Dunmore J, Lu B, Wang JW, Lee WC, Kamal A, Burrows F, Eckman C, Hutton M, Petrucelli L (2006) HSP induction mediates selective clearance of tau phosphorylated at proline-directed Ser/Thr sites but not KXGS (MARK) sites. ASEB J. 20:753-755

Dickey CA, Kamal A, Lundgren K, Klosak N, Bailey RM, Dunmore J, Ash P, Shoraka S, Zlatkovic J, Eckman CB, et al. (2007) The high-affinity HSP90-CHIP complex recognizes and selectively degrades phosphorylated tau client proteins. J Clin Invest. 117:648-658.

Donmez, G., Arun, A., Chung, C.Y., McLean, P.J., Lindquist, S. and Guarente, L (2012) SIRT1 protects against $\alpha$-synuclein ag- gregation by activating molecular chaperones. J. Neurosci., 32, 124-132.

Dou F, Netzer WJ, Tanemura K, Li F, Hartl FU, Takashima A, Gouras GK, Greengard P, $\mathrm{Xu} \mathrm{H}$ (2003) Chaperones increase association of tau protein with microtubules. Proc Natl Acad Sci 100:721-726

Drexler HC (2009) Synergistic apoptosis induction in leukemic cells by the phosphatase inhibitor salubrinal and proteasome inhibitors. PLoS One. 4:e4161

Duff K, Knight H, Refolo LM, Sanders S, Yu X, Picciano M, Malester B, Hutton M, Adamson J, Goedert M, et al. (2000) Characterization of pathology in transgenic mice over-expressing human genomic and cDNA tau transgenes. Neurobiol Dis. 7:87-98

Eichenbaum H (2004) Hippocampus: cognitive processes and neural representations that underlie declarative memory. Neuron. 44:109-120.

Ellis J (1987) Proteins as molecular chaperones. Nature. 328:378-379. 
Fang NN, Chan GT, Zhu M, Comyn SA, Persaud A, Deshaies RJ, Rotin D, Gsponer J, Mayor T (2014) Rsp5/Nedd4 is the main ubiquitin ligase that targets cytosolic misfolded proteins following heat stress. Nat. Cell. Biol., 16, 1227-1237.

Ferreiro E, Pereira CM (2012) Endoplasmic reticulum stress: a new playER in tauopathies. J Pathol 226(5):687-692

Georgieff I, Liem RKH, Mellado W, Nunez, J, Shelanski, ML (1991). High molecular weight tau: preferential localization in theperipheral nervous system. J. Cell Sci. 100: 5560.

Ghemrawi R, Pooya S, Lorentz S, Gauchotte G, Arnold C, Gueant JL et al. (2013) Decreased vitamin B12 availability induces ER stress through impaired SIRT1deacetylation of HSF1. Cell Death Dis. 4:e553

Giasson BI, Forman MS, Higuchi M, Golbe LI, Graves CL, Kotzbauer PT et al. (2003) Initiation and synergistic fibrillization of tau and alpha-synuclein. Science 300:636-640.

Glenner GG, Wong CW (1984) Alzheimer's disease: initial report of the purification and characterization of a novel cerebrovascular amyloid protein. Biochem Biophys Res Commun 120: 885-890.

Golde TE, Borchelt DR, Giasson BI, Lewis J (2013) Thinking laterally about neurodegenerative proteinopathies. J Clin Invest. 123:1847-1855

Gorbatyuk MS, Gorbatyuk OS (2013) The Molecular Chaperone GRP78/BiP as a Therapeutic Target for Neurodegenerative Disorders: A Mini Review. J Genet Syndr Gene Ther 4. pii: 128.

Götz J, Probst A, Spillantini MG, Schäfer T, Jakes R, Bürki K, Goedert M (1995) Somatodendritic localization and hyperphosphorylation of tau protein in transgenic mice expressing the longest human brain tau isoform. EMBO J. 14:1304-1313

Green M, Schuetz TJ, Sullivan EK, Kingston RE (1995) A heat shock-responsive domain of human HSF1 that regulates transcription activation domain function. Mol. Cell. Biol. 15:3354-3362

Grinberg, L. T., Rub, U., Ferretti, R. E., Nitrini, R., Farfel, J. M., Polichiso, L., Gierga, K., Jacob-Filho, W., and Heinsen, H. (2009). The dorsal raphe nucleus shows phosphotau neurofibrillary changes before the transentorhinal region in Alzheimer's disease. A precocious onset? Neuropathol. Appl. Neurobiol. 35, 406-416.

Guettouche T, Boellmann F, Lane WS, Voellmy R (2005) Analysis of phosphorylation of human heat shock factor 1 in cells experiencing a stress. BMC Biochem 6:4. 
Guo Q, Li H, Cole AL, Hur JY, Li Y, Zheng H (2013) Modeling Alzheimer's disease in mouse without mutant protein overexpression: cooperative and independent effects of $A \beta$ and tau. PLoS One. 8:e80706

Gustke N, Trinczek B, Biernat J, Mandelkow EM, Mandelkow E (1994) Domains of Tau- protein and interactions with microtubules. Biochemistry 33: 9511-9522

Haggerty T, Credle J, Rodriguez O, Wills J, Oaks AW, Masliah E et al. (2011) Hyperphosphorylated Tau in an $\alpha$-synuclein-overexpressing transgenic model of Parkinson's disease. Eur J Neurosci 33:1598-1610

Haitani Y, Shimoi H, Takagi H (2006) Rsp5 regulates ex- pression of stress proteins via post-translational modifica- tion of Hsf1 and Msn4 in Saccharomyces cerevisiae. FEBS Lett., 580, 3433-3438.

Haitani Y and Takagi H (2008) Rsp5 is required for the nuclear export of mRNA of HSF1 and MSN2/4 under stress conditions in Saccharomyces cerevisiae. Genes Cells, 13, $105-116$.

Hara T, Nakamura K, Matsui M, Yamamoto A, Nakahara Y, Suzuki-Migishima R, Yokoyama M, Mishima K, Saito I, Okano H, et al. (2006) Suppression of basal autophagy in neural cells causes neurodegenerative disease in mice. Nature. 441:885-889

Hershko A, Ciechanover A (1998) The ubiquitin system. Annu Rev Biochem. 67:425479.

Hetz C, Mollereau B (2014) Disturbance of endoplasmic reticulum proteostasis in neurodegenerative diseases. Nat Rev Neurosci 15:233-249

Hieronymus H, Lamb J, Ross KN, Peng XP, Clement C, Rodina A, Nieto M, Du J, Stegmaier K, Raj SM, et al. (2006) Gene expression signature-based chemical genomic prediction identifies a novel class of HSP90 pathway modulators. Cancer Cell. 10:321 330 .

Higashi T, Nakai A, Uemura Y, Kikuchi H, Nagata K (1995). Activation of heat shock factor 1 in rat brain during cerebral ischemia or after heat shock. Brain Res Mol Brain Res. 34:262-270.

Ho YS, Yang X, Lau JC, Hung CH, Wuwongse S, Zhang Q et al (2012) Endoplasmic reticulum stress induces tau pathology and forms a vicious cycle: implication in Alzheimer's disease pathogenesis. J Alzheimers Dis. 28:839-854

Höhfeld J, Cyr DM, Patterson C (2001) From the cradle to the grave: molecular chaperones that may choose between folding and degradation. EMBO Rep. 2:885-890. 
Holmberg CI, Hietakangas V, Mikhailov A, Rantanen JO, Kallio M, et al. (2001) Phosphorylation of serine 230 promotes inducible transcriptional activity of heat shock factor 1. EMBO J. 20:3800-3810

Homma S, Jin X, Wang G, Tu N, Min J, Yanasak N et al (2007) Demyelination, astrogliosis, and accumulation of ubiquitinated proteins, hallmarks of CNS disease in hsf1-deficient mice. J Neurosci 27:7974-7986

Hooper PL, Durham HD, Török Z, Hooper PL, Crul T, Vígh L (2016) The central role of heat shock factor 1 in synaptic fidelity and memory consolidation. Cell Stress Chaperones. 21:745-753

Hoover BR, Reed MN, Su J, Penrod RD, Kotilinek LA, Grant MK, Pitstick R, Carlson GA, Lanier LM, Yuan LL, et al (2010) Tau mislocalization to dendritic spines mediates synaptic dysfunction independently of neurodegeneration. Neuron 68:1067-1081

Hoozemans JJ, Veerhuis R, Van Haastert ES, Rozemuller JM, Baas F, Eikelenboom P, Scheper W (2005) The unfolded protein response is activated in Alzheimer's disease. Acta Neuropathol. 110:165-172

Hoozemans JJ, Van Haastert ES, Eikelenboom P, de Vos RA, Rozemuller JM, Scheper W (2007) Activation of the unfolded protein response in Parkinson's disease. Biochem Biophys Res Commun.354:707-711

Hoozemans JJ, van Haastert ES, Nijholt DA, Rozemuller AJ, Eikelenboom P, Scheper W (2009) The unfolded protein response is activated in pretangle neurons in Alzheimer's disease hippocampus. Am J Pathol. 174:1241-1251.

Hou J, Tang H, Liu Z, Österlund T, Nielsen J, Petranovic D (2014) Management of the endoplasmic reticulum stress by activation of the heat shock response in yeast. FEMS Yeast Res 14:481-494

Hunsberger HC, Weitzner DS, Rudy CC, Hickman JE, Libell EM, Speer RR et al (2015) Riluzole rescues glutamate alterations, cognitive deficits, and tau pathology associated with P301L tau expression. J Neurochem 135:381-394

Hyman BT, Van Hoesen GW, Damasio AR, Barnes CL (1984) Alzheimer's disease: Cell-specific pathology isolates the hippocampal formation. Science 225:1168-1170

Ihara Y, Nukina N, Miura R, Ogawara M (1986) Phosphorylated tau protein is integrated into paired helical filaments in Alzheimer's disease. J Biochem. 99:1807-1810

Ishizawa T, Mattila P, Davies P, Wang D, Dickson DW. Colocalization of tau and alphasynuclein epitopes in Lewy bodies. J Neuropathol Exp Neurol. 2003 Apr;62(4):389-97. 
Ittner LM, Ke YD, Delerue F, Bi M, Gladbach A, van Eersel J, Wölfing H, Chieng BC, Christie MJ, Napier IA, et al. (2010) Dendritic function of tau mediates amyloid-beta toxicity in Alzheimer's disease mouse models. Cell. 142:387-397

Jensen PH, Nielsen MS, Jakes R, Dotti CG, Goedert M (1998) Binding of alphasynuclein to brain vesicles is abolished by familial Parkinson's disease mutation. J Biol Chem. 273:26292-26924.

Jiang YQ, Wang XL, Cao XH, Ye ZY, Li L, Cai WQ (2013) Increased heat shock transcription factor 1 in the cerebellum reverses the deficiency of Purkinje cells in Alzheimer's disease. Brain Res. 1519:105-111.

Julien C, Tremblay C, Emond V, Lebbadi M, Salem N Jr, Bennett DA, Calon F (2009) Sirtuin 1 reduction parallels the accumulation of tau in Alzheimer disease. J Neuropathol Exp Neurol. 68:48-58.

Kalmar B, Greensmith L. Activation of the heat shock response in a primary cellular model of motoneuron neurodegeneration - evidence for neuroprotective and neurotoxic effects. Cell. Mol. Biol. Lett. 2009; 14:319-335.

Kato H, Nakajima S, Saito Y, Takahashi S, Katoh R, Kitamura M (2012) mTORC1 serves ER stress-triggered apoptosis via selective activation of the IRE1-JNK pathway. Cell Death Differ 19:310-320

Keller JN, Hanni KB, Markesbery WR (2000) Impaired proteasome function in Alzheimer's disease. J Neurochem. 75:436-439.

Khurana V, Elson-Schwab I, Fulga TA, Sharp KA, Loewen CA, Mulkearns E, Tyynelä J, Scherzer CR, Feany MB (2010) Lysosomal dysfunction promotes cleavage and neurotoxicity of tau in vivo. PLoS Genet. 6:e1001026.

Kieran D, Kalmar B, Dick JR, Riddoch-Contreras J, Burnstock G, Greensmith L (2004) Treatment with arimoclomol, a coinducer of heat shock proteins, delays disease progression in ALS mice. Nat Med. 10:402-405.

Kim D, Nguyen MD, Dobbin MM, Fischer A, Sananbenesi F, Rodgers JT, Delalle I, Baur JA, Sui G, Armour SM, Puigserver P, Sinclair DA, Tsai LH (2007) SIRT1 deacetylase protects against neurodegeneration in models for Alzheimer's disease and amyotrophic lateral sclerosis. EMBO J. 26:3169-3179.

Kim E, Wang B, Sastry N, Masliah E, Nelson PT, Cai H et al. (2016) NEDD4-mediated HSF1 degradation underlies $\alpha$-synucleinopathy. Hum Mol Genet 25:211-222

Kline MP, Morimoto RI (1997) Repression of the heat shock factor 1 transcriptional activation domain is modulated by constitutive phosphorylation. Mol. Cell Biol. $17: 2107-2115$ 
Klucken J, Shin Y, Masliah E, Hyman BT, McLean PJ (2004) Hsp70 Reduces alphaSynuclein Aggregation and Toxicity. J Biol Chem. 279:25497-25502.

Klucken J, Ingelsson M, Shin Y, Irizarry MC, Hedley-Whyte ET, Frosch M (2006). Clinical and biochemical cor- relates of insoluble alpha-synuclein in dementia with Lewy bodies. Acta Neuropathol 111:101-108

Kokhan VS, Afanasyeva MA, Van'kin GI (2012) $\alpha$-Synuclein knockout mice have cognitive impairments.Behav Brain Res. 231:226-230

Kondo N, Katsuno M, Adachi H, Minamiyama M, Doi H, Matsumoto S, Miyazaki Y, Iida M, Tohnai G, Nakatsuji H, et al. (2013) Heat shock factor-1 influences pathological lesion distribution of polyglutamine-induced neurodegeneration. Nat Commun. 4:1405

Kosik KS, Crandall, JE, Mufson, EJ, Neve RL (1989) Tau in situ hybridization in normal and Alzheimer brain: localization in the somatodendritic compartment. Ann. Neurol. 26: $352-361$

Kotzbauer PT, Giasson BI, Kravitz AV, Golbe LI, Mark MH, Trojanowski JQ, Lee VM (2004) Fibrillization of alpha-synuclein and tau in familial Parkinson's disease caused by the A53T alpha-synuclein mutation. Exp Neurol. 187: 279-288.

Kourtis N, Moubarak RS, Aranda-Orgilles B, Lui K, Aydin IT, Trimarchi T, Darvishian F, Salvaggio C, Zhong J, Bhatt K, et al. (2015) FBXW7 modulates cellular stress response and metastatic potential through HSF1 post-translational modification. Nat Cell Biol. 17:322-332.

Kumar S, Harvey KF, Kinoshita M, Copeland NG, Noda M, Jenkins, NA (1997). cDNA cloning, expression analysis, and mapping of the mouse Nedd4 gene. Genomics 40: 435443.

Kwak YD, Wang B, Li JJ, Wang R, Deng Q, Diao S, Chen Y, Xu R, Masliah E, Xu H. et al. (2012) Upregulation of the E3 ligase NEDD4-1 by oxidative stress degrades IGF-1 receptor protein in neurodegeneration. J. Neurosci., 32, 10971-10981.

Leak RK (2014) Heat shock proteins in neurodegenerative disorders and aging. J Cell Commun Signal. 8:293-310.

LeDoux JE, Cicchetti P, Xagoraris A, Romanski LM (1990) The lateral amygdaloid nucleus: sensory interface of the amygdala in fear conditioning. J Neurosci.10:10621069.

Lee JH, Won SM, Suh J, Son SJ, Moon GJ, Park UJ et al. (2010) Induction of the unfolded protein response and cell death pathway in Alzheimer's disease, but not in aged Tg2576 mice. Exp Mol Med 42:386-394. 
Lin X., Parisiadou L, Gu XL, Wang L., Shim H., Sun L., Xie C., Long CX, Yang WJ, Ding J. et al. (2009) Leucine-rich repeat kinase 2 regulates the progression of neuropathology in- duced by Parkinson's-disease-related mutant alpha- synuclein. Neuron, 64, 807-827.

Lindersson E., Beedholm R., Hojrup P., Moos T., Gai W. P., Hendil K. B., Jensen P. H. (2004) Proteasomal inhibition by $\alpha$-synuclein filaments and oligomers. J. Biol. Chem. 279:12924-12934

Lindquist, S. (1986) The heat-shock response. Annu. Rev. Bio- chem., 55, 1151-1191.

Littlefield O, Nelson HCM. 1999. A new use for the 'wing' of the 'winged' helix-turnhelix motif in the HSF-DNA cocrystal. Nat. Struct. Biol. 6:464-470

Liu AY, Mathur R., Mei N, Langhammer CG, Babiarz B and Firestein BL (2011) Neuroprotective drug riluzole amplifies the heat shock factor 1 (HSF1)- and glutamate transporter 1 (GLT1)-dependent cytoprotective mechanisms for neuronal survival. J. Biol. Chem., 286, 2785-2794.

Liu WK, Dickson DW, Yen SH (1993) Heterogeneity of tau proteins in Alzheimer's disease. Evidence for increased expression of an isoform and preferential distribution of a phosphorylated isoform in neurites. Am J Pathol. 142:387-394.

Liu Y, Chang A (2008) Heat shock response relieves ER stress. EMBO J 27(7):10491059

Lu RC, Tan MS, Wang H, Xie AM, Yu JT, Tan L (2014) Heat shock protein 70 in Alzheimer's disease. Biomed Res Int. 435203

Luo W, Dou F, Rodina A, Chip S, Kim J, Zhao Q, Moulick K, Aguirre J, Wu N, Greengard P, Chiosis G (2007) Roles of heat-shock protein 90 in maintaining and facilitating the neurodegenerative phenotype in tauopathies. Proc Natl Acad Sci. 104:9511-9516

Ly PT, Cai F, Song W (2011) Detection of neuritic plaques in Alzheimer's disease mouse model. J Vis Exp. (53). pii: 2831

Ma QL, Zuo X, Yang F, Ubeda OJ, Gant DJ, Alaverdyan M, Teng E, Hu S, Chen PP, Maiti P, Teter B, Cole GM, Frautschy SA (2013) Curcumin suppresses soluble tau dimers and corrects molecular chaperone, synaptic, and behavioral deficits in aged human tau transgenic mice. J Biol Chem. 288:4056-4065.

Ma T, Trinh MA, Wexler AJ, Bourbon C, Gatti E, Pierre P, Cavener DR, Klann E (2013) Suppression of eIF2 $\alpha$ kinases alleviates Alzheimer's disease-related plasticity and memory deficits. Nat Neurosci. 16: 1299-1305 
Magrané J, Smith RC, Walsh K, Querfurth HW (2004) Heat shock protein 70 participates in the neuroprotective response to intracellularly expressed beta-amyloid in neurons. $\mathrm{J}$ Neurosci. 24:1700-1706.

Marcuccilli CJ, Mathur SK, Morimoto RI, Miller RJ (1996) Regulatory differences in the stress response of hippocampal neurons and glial cells after heat shock. J Neurosci. $16: 478-485$.

Mattson MP, Akbari Y, LaFerla FM (2003) Triple-transgenic model of Alzheimer's disease with plaques and tangles: intracellular Abeta and synaptic dysfunction. Neuron. 39:409-421

Matus S, Glimcher LH, Hetz C (2011) Protein folding stress in neurodegenerative diseases: a glimpse into the ER. Curr Opin Cell Biol. 23:239-252.

McLean PJ, Kawamata H, Ribich S, Hyman BT (2000) Membrane association and protein conformation of alpha-synuclein in intact neurons. Effect of Parkinson's diseaselinked mutations. J Biol Chem. 275:8812-8816.

McMillan DR, Xiao X, Shao L, Graves K, Benjamin IJ (1998) Targeted disruption of heat shock transcription factor 1 abolishes thermotolerance and protection against heatinducible apoptosis. J. Biol. Chem. 273:7523-7528

Medina, M. and Castro, A. (2008) Glycogen synthase kinase-3 (GSK-3) inhibitors reach the clinic. Curr. Opin. Drug Discov. Devel. 11:533-543

Meier S, Bell M, Lyons DN, Ingram A, Chen J, Gensel JC, Zhu H, Nelson PT,Abisambra JF (2015) Identification of Novel Tau Interactions with Endoplasmic Reticulum Proteins in Alzheimer's Disease Brain. J Alzheimers Dis. 48:687-702

Merrill DA, Masliah E, Roberts JA, McKay H, Kordower JH, Mufson EJ, Tuszynski MH (2011) Association of early experience with neurodegeneration in aged primates.

Neurobiol Aging. 32:151-156

Min SW, Cho SH, Zhou Y, Schroeder S, Haroutunian V, Seeley WW, Huang EJ, Shen Y, Masliah E, Mukherjee C, Meyers D, Cole PA, Ott M, Gan L (2010)

Acetylation of tau inhibits its degradation and contributes to tauopathy. Neuron. 67:953966

Mocanu MM, Nissen A, Eckermann K, Khlistunova I, Biernat J, Drexler D et al. (2008) The potential for beta-structure in the repeat domain of tau protein determines aggregation, synaptic decay, neuronal loss, and coassembly with endogenous Tau in inducible mouse models of tauopathy. J Neurosci. 28:737-748. 
Moon IS, Park IS, Schenker LT, Kennedy MB, Moon JI, Jin I (2001) Presence of both constitutive and inducible forms of heat shock protein 70 in the cerebral cortex and hippocampal synapses. Cereb Cortex. 11:238-248.

Moran PM, Higgins LS, Cordell B, Moser PC (1995) Age-related learning deficits in transgenic mice expressing the 751-amino acid isoform of human $\beta$-amyloid precursor protein. Proc Natl Acad Sci USA 92:5341-5345

Moreno JA, Halliday M, Molloy C, Radford H, Verity N, Axten JM, Ortori CA, Willis AE, Fischer PM, Barrett DA, et al. (2013) Oral treatment targeting the unfolded protein response prevents neurodegeneration and clinical disease in prion-infected mice. Sci Transl Med. 5:206

Mori K, Sant A, Kohno K, Normington K, Gething M J, Sambrook J F (1992) A 22 bp cis- acting element is necessary and sufficient for the induction of the yeast KAR2 (BiP) gene by unfolded proteins. EMBO J 11: 2583-2593.

Morimoto RI (1998) Regulation of the heat shock transcrip- tional response: cross talk between a family of heat shock factors, molecular chaperones, and negative regulators. Genes Dev., 12, 3788-3796.

Morimoto RI (2008) Proteotoxicstressandinduciblechaper- one networks in neurodegenerative disease and aging. Genes Dev., 22, 1427-1438.

Morris M, Maeda S, Vossel K, Mucke L (2011) The many faces of tau. Neuron. 70:410426

Moussaud S, Jones DR, Moussaud-Lamodière EL, Delenclos M, Ross OA, McLean PJ (2014) Alpha-synuclein and tau: teammates in neurodegeneration? Mol Neurodegener. 9:43.

Muchowski PJ, Wacker JL (2005) Modulation of neurodegeneration by molecular chaperones. Nat Rev Neurosci. 6:11-22

Mukaetova-Ladinska EB, Hurt J, Jakes R, Xuereb J, Honer WG, Wischik CM (2000) Alpha- synuclein inclusions in Alzheimer and Lewy body diseases. J. Neuropathol. Exp. Neurol. 59:408-417.

Murata S, Minami Y, Minami M, Chiba T, Tanaka K (2001) CHIP is a chaperonedependent E3 ligase that ubiquitylates unfolded protein. EMBO Rep. 2:1133-1138

Myeku N, Clelland CL, Emrani S, Kukushkin NV, Yu WH, Goldberg AL, Duff KE (2016) Tau-driven 26S proteasome impairment and cognitive dysfunction can be prevented early in disease by activating cAMP-PKA signaling. Nat Med. 22:46-53. 
Neef DW, Turski ML, Thiele DJ (2010) Modulation of heat shock transcription factor 1 as a therapeutic target for small molecule intervention in neurodegenerative disease.

PLoS Biol. 8:e1000291.

Neef DW, Jaeger AM, Thiele DJ (2011) Heat shock transcription factor 1 as a therapeutic target in neurodegenerative diseases. Nat Rev Drug Discov 10:930-944

Neef DW, Jaeger AM, Gomez-Pastor R, Willmund F, Frydman J, Thiele DJ (2014) A direct regulatory interaction between chaperonin $\mathrm{TRiC}$ and stress-responsive transcription factor HSF1. Cell Rep. 9:955-966.

Nelson PT, Abner EL, Schmitt FA, Kryscio RJ, Jicha GA, Santacruz K et al. (2009) Brains with medial temporal lobe neurofibrillary tangles but no neuritic amyloid plaques are a diagnostic dilemma but may have pathogenetic aspects distinct from Alzheimer disease. J Neuropathol Exp Neurol. 68:774-784.

Nelson PT, Kryscio RJ, Jicha GA, Abner EL, Schmitt FA, Xu LO, Cooper G, Smith CD, Markesbery WR (2009) Relative preservation of MMSE scores in autopsy-proven dementia with Lewy bodies. Neurology, 73, 1127-1133.

Nelson PT, Abner EL, Schmitt FA, Kryscio RJ, Jicha GA, Smith CD et al. (2010) Modeling the association between 43 different clinical and pathological variables and the severity of cognitive impairment in a large autopsy cohort of elderly persons. Brain Pathol. 20:66-79

Nelson PT, Alafuzoff I, Bigio EH, Bouras C, Braak H, Cairns NJ et al. (2012) Correlation of Alzheimer disease neuropathologic changes with cognitive status: a review of the literature. J Neuropathol Exp Neurol. 71:362-381.

Nijholt DA, Van Haastert ES, Rozemuller AJ, Scheper W, Hoozemans JJ (2012) The unfolded protein response is associated with early tau pathology in the hippocampus of tauopathies. J Pathol 226:693-702

Nijholt DA, Nölle A, van Haastert ES, Edelijn H, Toonen RF, Hoozemans JJ, Scheper W (2013) Unfolded protein response activates glycogen synthase kinase-3 via selective lysosomal degradation. Neurobiol Aging. 34:1759-1771

Nixon RA, Cataldo AM, Paskevich PA, Hamilton DJ, Wheelock TR, Kanaley-Andrews L (1992) The lysosomal system in neurons. Involvement at multiple stages of Alzheimer's disease pathogenesis. Ann N Y Acad Sci. 674:65-88.

Oddo S, Caccamo A, Shepherd JD, Murphy MP, Golde TE, Kayed R, Metherate R, Mattson MP, Akbari Y, LaFerla FM (2003) Triple-transgenic model of Alzheimer's disease with plaques and tangles: intracellular Abeta and synaptic dysfunction. Neuron. 39:409-421. 
Ogata M, Hino S, Saito A, Morikawa K, Kondo S, Kanemoto S, Murakami T, Taniguchi M, Tanii I, Yoshinaga K, et al. (2006) Autophagy is activated for cell survival after endoplasmic reticulum stress. Mol Cell Biol. 26:9220-9231

Oh S, Hong HS, Hwang E, Sim HJ, Lee W, Shin SJ, Mook-Jung I (2005) Amyloid peptide attenuates the proteasome activity in neuronal cells. Mech Ageing Dev. 126:1292-1299

Ohsumi Y (2014) Historical landmarks of autophagy research. Cell Res. 24:9-23

Outeiro TF, Klucken J, Strathearn KE, Liu F, Nguyen P, Rochet JC, Hyman BT, McLean PJ (2006) Small heat shock proteins protect against alpha-synuclein-induced toxicity and aggregation. Biochem Biophys Res Commun. 351:631-638

Papassotiropoulos A, Bagli M, Kurz A, Kornhuber J, Förstl H, et al. (2000) A genetic variation of cathepsin D is a major risk factor for Alzheimer's disease. Ann Neurol 47: 399-403.

Paris D, Ganey NJ, Laporte V, Patel NS, Beaulieu-Abdelahad D, Bachmeier C, March A, Ait-Ghezala G, Mullan MJ (2010) Reduction of beta-amyloid pathology by celastrol in a transgenic mouse model of Alzheimer's disease. J Neuroinflammation. 7:17

Perrin V, Régulier E, Abbas-Terki T, Hassig R, Brouillet E, Aebischer P, Luthi-Carter R, Déglon N (2007) Neuroprotection by Hsp104 and Hsp27 in lentiviral-based rat models of Huntington's disease. Mol Ther. 15:903-911

Petrucelli L, Dickson D, Kehoe K, Taylor J, Snyder H, Grover A, De Lucia M, McGowan E, Lewis J, Prihar G, et al. (2004) CHIP and Hsp70 regulate tau ubiquitination, degradation and aggregation. Hum Mol Genet. 13:703-714.

Phillips RG, LeDoux JE (1992) Differential contribution of amygdala andhippocampus to cued and contextual fear conditioning. Behav Neu-rosci 106:274-285.

Pickford F, Masliah E, Britschgi M, Lucin K, Narasimhan R, Jaeger PA, Small S, Spencer B, Rockenstein E, Levine B, et al. (2008) The autophagy-related protein beclin 1 shows reduced expression in early Alzheimer disease and regulates amyloid beta accumulation in mice. J Clin Invest. 118:2190-2199

Pierce A, Podlutskaya N, Halloran JJ, Hussong SA, Lin PY, Burbank R, Hart MJ, Galvan $\mathrm{V}$ (2013) Over-expression of heat shock factor 1 phenocopies the effect of chronic inhibition of TOR by rapamycin and is sufficient to ameliorate Alzheimer's-like deficits in mice modeling the disease. J Neurochem. 124:880-893

Pirkkala L, Alastalo TP, Zuo X, Benjamin Ij, Sistonen L (2000) Disruption of heat shock factor 1 reveals an essential role in the ubiquitin proteolytic pathway. Mol. Cell Biol., 20, 733. 
Poch C, Fuentemilla L, Barnes GR, Düzel E (2011) Hippocampal theta-phase modulation of replay correlates with configural-relational short-term memory performance. $\mathrm{J}$

Neurosci. 31:7038-7042

Polydoro M, Dzhala VI, Pooler AM, Nicholls SB, McKinney AP, Sanchez L, Pitstick R, Carlson GA, Staley KJ, Spires-Jones TL, et al. (2014) Soluble pathological tau in the entorhinal cortex leads to presynaptic deficits in an early Alzheimer's disease model. Acta Neuropathol. 127: 257-270.

Polymeropoulos MH, Lavedan C, Leroy E, Ide SE, Dehejia A, Dutra A, Pike B, Root H, Rubenstein J, Boyer R, et al. (1997) Mutation in the alpha-synuclein gene identified in families with Parkinson's disease. Science. 276:2045-2047.

Pooler AM, Phillips EC, Lau DH, Noble W, Hanger DP (2013) Physiological release of endogenous tau is stimulated by neuronal activity. EMBO Rep. 14:389-394

Postina R, Schroeder A, Dewachter I, Bohl J, Schmitt U, Kojro E, Prinzen C, Endres K, Hiemke C, Blessing M, et al. (2004) A disintegrin-metalloproteinase prevents amyloid plaque formation and hippocampal defects in an Alzheimer disease mouse model. J Clin Invest. 113:1456-1464.

Rabindran SK, Haroun RI, Clos J, Wisniewski J, Wu C (1993) Regulation of heat shock factor trimer formation: role of a conserved leucine zipper. Science 259:230-234

Ramsden M, Kotilinek L, Forster C, Paulson J, McGowan E, SantaCruz K, et al. (2005) Age- dependent neurofibrillary tangle formation, neuron loss, and memory impairment in a mouse model of human tauopathy (P301L). J Neurosci 25:10637-10647

Rane MJ, Pan Y, Singh S, Powell DW, Wu R, Cummins T, Chen Q, McLeish KR, Klein JB (2003) Heat shock protein 27 controls apoptosis by regulating Akt activation. J Biol Chem. 278:27828-27835

Rapoport M, Dawson HN., Binder LI, Vitek MP, Ferreira A (2002) Tau is essential to $\beta$ amyloid-induced neurotoxicity. Proc Natl Acad Sci U S A. 99: 6364-6369

Raychaudhuri S, Loew C, Körner R, Pinkert S, Theis M, Hayer-Hartl M, Buchholz F, Hartl FU (2014) Interplay of acetyltransferase EP300 and the proteasome system in regulating heat shock transcription factor 1. Cell. 156:975-985

Resende R, Ferreiro E, Pereira C, Oliveira CR (2008) ER stress is involved in Abetainduced GSK-3beta activation and tau phosphorylation. Neurosci Res 86:2091-2099

Ritossa F (1962). "A new puffing pattern induced by temperature shock and DNP in drosophila". Experientia. 18: 571-573 
Rizzu P, Van Swieten JC, Joosse M, Hasegawa M, Stevens M, Tibben A, et al. (1999) High prevalence of mutations in the microtubule-associated protein tau in a population study of frontotemporal dementia in The Netherlands. Am J Hum Genet 64:414-421.

Roberson ED, Scearce-Levie K, Palop JJ, Yan F, Cheng IH, Wu T, et al. (2007) Reducing endogenous tau ameliorates amyloid $\beta$-induced deficits in an Alzheimer's disease mouse model. Science 316:750-754

Sakagami Y, Kudo T, Tanimukai H, Kanayama D, Omi T, Horiguchi K, Okochi M, Imaizumi K, Takeda M (2013) Involvement of endoplasmic reticulum stress in tauopathy. Biochem Biophys Res Commun. 430:500-504.

Saman S, Kim W, Raya M, Visnick Y, Miro S, Saman S, Jackson B, McKee AC, Alvarez VE, Lee NC, Hall GF (2012) Exosome-associated tau is secreted in tauopathy models and is selectively phosphorylated in cerebrospinal fluid in early Alzheimer disease. J Biol Chem. 287:3842-3849

Sampalo-Marques B and Ludovico P (2015) Sirtuins and proteolytic systems: implications for pathogenesis of synu- cleinopathies. Biomolecules, 5, 735-757. Sanders MJ, Wiltgen BJ, Fanselow MS (2003) The place of the hippocampus in fear conditioning. Eur J Pharmacol. 463:217-223.

Santacruz K, Lewis J, Spires T, Paulson J, Kotilinek L, Ingelsson M, Guimaraes A, DeTure M, Ramsden M, McGowan E, et al. (2005) Tau suppression in a neurodegenerative mouse model improves memory function. Science. 309:476-481.

Santa-María I, Pérez M, Hernández F, Avila J, Moreno FJ (2006) Characteristics of the binding of thioflavin S to tau paired helical filaments. J Alzheimers Dis. 9: 279-285.

Sato H, Kato T, Arawaka S (2013) The role of Ser129 phosphorylation of $\alpha$-synuclein in neurodegeneration of Parkinson's disease: a review of in vivo models. Rev Neurosci. 24: $115-123$

Saxena S, Caroni P (2011) Selective neuronal vulnerability in neurodegenerative diseases: from stressor thresholds to degeneration. Neuron. 71:35-48

Schaffar G, Breuer P, Boteva R, Behrends C, Tzvetkov N, Strippel N, Sakahira H, Siegers K, Hayer-Hartl M, Hartl FU (2004) Cellular toxicity of polyglutamine expansion proteins: mechanism of transcription factor deactivation. Mol Cell.15:95-105.

Schlesinger MJ (1990) Heat shock proteins. J Biol Chem. 265:12111-12114

Schmitt FA, Nelson PT, Abner E, Scheff S, Jicha GA, Smith C, Cooper G, Mendiondo M, Danner DD, Van Eldik LJ et al. (2012) University of Kentucky Sanders-Brown healthy brain aging volunteers: donor characteristics, procedures and neuropathology. Curr. Alzheimer Res., 9, 724-733 
Schröder M, Kaufman RJ (2005) The mammalian unfolded protein response. Annu Rev Biochem 74:739-789

Scoville WB, Milner B (1957) Loss of recent memory after bilateral hippocampal lesions. J Neurol Neurosurg Psychiatry. 20:11-21.

Shcherbik N, Kee Y, Lyon N, Huibregtse JM, Haines DS (2004) A single PXY motif located within the carboxyl ter- minus of Spt23p and Mga2p mediates a physical and functional interaction with ubiquitin ligase Rsp5p. J. Biol. Chem., 279, 53892-53898.

Shen HY, He JC, Wang Y, Huang QY, Chen JF (2005) Geldanamycin induces heat shock protein 70 and protects against MPTP-induced dopaminergic neurotoxicity in mice. $\mathrm{J}$ Biol Chem. 280:39962-39969

Shi Y, Mosser DD, Morimoto RI (1998) Molecular cha- perones as HSF1-specific transcriptional repressors. Genes Dev., 12, 654-666.

Shimura H, Miura-Shimura Y, Kosik KS. Binding of tau to heat shock protein 27 leads to decreased concentration of hyperphosphorylated tau and enhanced cell survival. J. Biol. Chem. 279, 17957-17962 (2004).

Shipton OA, Leitz JR, Dworzak J, Acton CE, Tunbridge EM, Denk F, et al. (2011) Tau protein is required for amyloid \{beta\}-induced impairment of hippocampal long-term potentiation. J Neurosci 31:1688-1692

Spatara ML Robinson AS (2010) Transgenic mouse and cell culture models demonstrate a lack of mechanistic connection between endoplasmic reticulum stress and tau dysfunction. J Neurosci Res 88:1951-1961

Speese SD, Trotta N, Rodesch CK, Aravamudan B, Broadie K (2003) The ubiquitin proteasome system acutely regulates presynaptic protein turnover and synaptic efficacy. Curr Biol. 13:899-910.

Spillantini MG, Goedert M, Crowther RA, Murrell JR, Farlow MR, Ghetti B (1997) Familial multiple system tauopathy with presenile dementia: a disease with abundant neuronal and glial tau filaments. Proc Natl Acad Sci U S A. 94:4113-4118.

Spillantini MG, Goedert M (1998). Tau protein pathology in neurodegenerative diseases. Trends Neurosci. 21:428-433.

Spires TL, Orne JD, SantaCruz K, Pitstick R, Carlson GA, Ashe KH, Hyman BT (2006) Region-specific dissociation of neuronal loss and neurofibrillary pathology in a mouse model of tauopathy. Am J Pathol. 168:1598-1607.

Sullivan EK, Weirich CS, Guyon JR, Sif S, Kingston RE (2001) Transcriptional activation domains of human heat shock factor 1 recruit human SWI/SNF. Mol. Cell. 
Biol. 21:5826-37

Sun F, Anantharam V, Zhang D, Latchoumycandane C, Kanthasamy A, Kanthasamy AG (2006) Proteasome inhibitor MG-132 induces dopaminergic degeneration in cell culture and animal models. Neurotoxicology 27:807-815

Tai HC, Serrano-Pozo A, Hashimoto T, Frosch MP, Spires-Jones TL, Hyman BT (2012) The synaptic accumulation of hyperphosphorylated tau oligomers in Alzheimer disease is associated with dysfunction of the ubiquitin-proteasome system. Am J Pathol. 181:14261435

Takeda S, Wegmann S, Cho H, DeVos SL, Commins C, Roe AD, Nicholls SB, Carlson GA, Pitstick R, Nobuhara CK, et al. (2015) Neuronal uptake and propagation of a rare phosphorylated high-molecular-weight tau derived from Alzheimer's disease brain. Nat Commun. 6:8490.

Takeuchi H, Iba M, Inoue H, Higuchi M, Takao K, Tsukita K, Karatsu Y, Iwamoto Y, Miyakawa T, Suhara T, et al. (2011) P301S mutant human tau transgenic mice manifest early symptoms of human tauopathies with dementia and altered sensorimotor gating. PLoS One. 6:e21050.

Tardiff DF, Jui NT, Khurana V, Tambe MA, Thompson ML, Chung CY, Kamadurai HB, Kim HT, Lancaster AK, Caldwell KA. et al. (2013) Yeast reveal a "druggable" Rsp5/Nedd4 network that ameliorates $\alpha$-synuclein toxicity in neurons. Science, 342, 979983.

Tashiro K, Hasegawa M, Ihara Y, Iwatsubo T (1997) Somatodendritic localization of phosphorylated tau in neonatal and adult rat cerebral cortex. Neuroreport. 8:2797-2801.

Tofaris, G.K., Kim, H.T., Hourez, R., Jung, J.W., Kim, K.P. and Goldberg, A.L. (2011) Ubiquitin ligase Nedd4 promotes alpha-synuclein degradation by the endosomallysosomal pathway. Proc. Natl. Acad. Sci. U S A, 108, 17004-17009.

Tortosa E, Santa-Maria I, Moreno F, Lim F, Perez M, Avila J (2009) Binding of Hsp90 to tau promotes a conformational change and aggregation of tau protein. J Alzheimers Dis. 17:319-325.

Tóth ME1, Szegedi V, Varga E, Juhász G, Horváth J, Borbély E, Csibrány B, Alföldi R, Lénárt N, Penke B, et al. (2013) Overexpression of Hsp27 ameliorates symptoms of Alzheimer's disease in APP/PS1 mice. Cell Stress Chaperones.18:759-771

Uchida S, Hara K, Kobayashi A, Fujimoto M, Otsuki K, Yamagata H, Hobara T, Abe N, Higuchi F, Shibata T, et al (2011) Impaired hippocampal spinogenesis and neurogenesis and altered affective behavior in mice lacking heat shock factor 1. Proc Natl Acad Sci. 108:1681-1686 
Unterberger U, Höftberger R, Gelpi E, Flicker H, Budka H, Voigtländer T (2006) Endoplasmic reticulum stress features are prominent in Alzheimer disease but not in prion diseases in vivo. J Neuropathol Exp Neurol 65:348-357.

Verma P, Pfister JA, Mallick S, D'Mello, SR (2014) HSF1 protects neurons through a novel trimerization- and HSP-in- dependent mechanism. J. Neurosci., 34, 1599-1612.

Vigh L, Literati PN, Horvath I, Torok Z, Balogh G, Glatz A, Kovács E, Boros I, Ferdinándy P, Farkas B, et al. (1997) Bimoclomol: a nontoxic, hydroxylamine derivative with stress protein-inducing activity and cytoprotective effects. Nat Med.

1997;3(10):1150-1154

Vogelsberg-Ragaglia V, Bruce J, Richter-Landsberg C, Zhang B, Hong M, Trojanowski JQ, et al. (2000) Distinct FTDP-17 missense mutations in tau produce tau aggregates and other pathological phenotypes in transfected CHO cells. Mol Biol Cell 27:4093-4104.

Voss OH, Batra S, Kolattukudy SJ, Gonzalez-Mejia ME, Smith JB, Doseff AI (2007) Binding of caspase-3 prodomain to heat shock protein 27 regulates monocyte apoptosis by inhibiting caspase-3 proteolytic activation. J Biol Chem. 282:25088-25099.

Wang B, Liu Y, Huang L, Chen J, Li JJ, Wang R, et al. (2016) A CNS-permeable Hsp90 inhibitor rescues synaptic dysfunction and memory loss in APP-overexpressing Alzheimer's mouse model via an HSF1-mediated mechanism. Mol Psychiatry. doi: 10.1038/mp.2016.104. [Epub ahead of print]

Wang X, Khaleque MA, Zhao MJ, Zhong R, Gaestel M, Calderwood SK (2006) Phosphorylation of HSF 1 by MAPK-activated protein kinase 2 on serine 121, inhibits transcriptional activity and promotes HSP90 binding. J Biol Chem 281:782-791

Wang XZ, Lawson B, Brewer JW, Zinszner H, Sanjay A, Mi LJ, et al. (1996) Signals from the stressed endoplasmic reticulum induce C/EBP-homologous protein (CHOP/GADD153). Mol Cell Biol 16:4273-4280

Weindling E, Bar-Nun S (2015) Sir2 links the unfolded protein response and the heat shock response in a stress response network. Biochem Biophys Res Commun. 457:473478

Westerheide SD, Anckar J, Stevens SM Jr, Sistonen L, Morimoto RI (2009) Stressinducible regulation of heat shock factor 1 by the deacetylase SIRT1. Science. 323:10631066.

Wiep Scheper, Jeroen J. M. Hoozemans (2015) The unfolded protein response in neurodegenerative diseases: a neuropathological perspective. Acta Neuropathol 130: $315-331$. 
Wilhelmus MM, Boelens WC, Otte-Höller I, Kamps B, de Waal RM, Verbeek MM (2006) Small heat shock proteins inhibit amyloid-beta protein aggregation and cerebrovascular amyloid-beta protein toxicity. Brain Res. 1089:67-78

Wu G, Wang X, Feng X, Zhang A, Li J, Gu K, Huang J, Pang S, Dong H, Gao H, Yan B (2011) Altered expression of autophagic genes in the peripheral leukocytes of patients with sporadic Parkinson's disease. Brain Res. 1394:105-111

Wu JW, Hussaini SA, Bastille IM, Rodriguez GA, Mrejeru A, Rilett K, Sanders DW, Cook C, Fu H, Boonen RA, et al. (2016) Neuronal activity enhances tau propagation and tau pathology in vivo. Nat Neurosci. 19:1085-1092

Xiao H, Perisic O, Lis JT. 1991. Cooperative binding of Drosophila heat shock factor to arrays of a conserved 5 bp unit. Cell 64:585-93

Xu P, Duong DM, Seyfried NT, Cheng D, Xie Y, Robert J et alQuantitative proteomics reveals the function of unconventional ubiquitin chains in proteasomal degradation. Cell 2009; 137: 133-145

Yang B, and Kumar S (2010). Nedd4 and Nedd4-2: closely related ubiquitin-protein ligases with distinct physiological functions. Cell Death Differ 17, 68-77.

Yang J, Bridges K, Chen KY, Liu AY (2008) Riluzole increases the amount of latent HSF1 for an amplified heat shock response and cytoprotection. PLoS One 3:e2864

Yoshiyama Y, Higuchi M, Zhang B, Huang SM, Iwata N, Saido TC, Maeda J, Suhara T, Trojanowski JQ, Lee VM (2007) Synapse loss and microglial activation precede tangles in a P301S tauopathy mouse model. Neuron. 53:337-351.

Zhao Y, Gong S, Shunmei E, Zou J (2009) Induction of macroautophagy by heat. Mol Biol Rep 36:2323-2327

Zhu X, Cheng M, Peng M, Xiao X, Yao S, Zhang X (2008) xd. Behav Brain Res. 193:225-229.

Zinszner H, Kuroda M, Wang X, Batchvarova N, Lightfoot RT, Remotti H, et al. (1998) CHOP is implicated in programmed cell death in response to impaired function of the endoplasmic reticulum. Genes Dev 12:982-995.

Zou J, Guo Y, Guettouche T, Smith DF, Voellmy R (1998) Repression of heat shock transcription factor HSF1 ac- tivation by HSP90 (HSP90 complex) that forms a stresssensi- tive complex with HSF1. Cell, 94, 471-480.

Zourlidou A, Gidalevitz T, Kristiansen M, Landles C, Woodman B, Wells DJ, Latchman DS, de Belleroche J, Tabrizi SJ, Morimoto RI, et al. (2007) Hsp27 overexpression in the 
R6/2 mouse model of Huntington's disease: chronic neurodegeneration does not induce Hsp27 activation. Hum Mol Genet. 16:1078-1090. 


\section{VITA}

Eunhee Kim was born in Seoul, Korea in 1988. She received her Bachelor of Science degree in Life Science from Hanyang University in Seoul, Korea in 2012. She joined the neuroscience track of interdisciplinary program at The University of Tennessee Health Science Center in August 2012. She began her research project in the laboratory of Dr. Francesca-Fang Liao in July 2013 and has conducted research involving molecular mechanisms of neurodegenerative diseases.

Ms. Kim's publications include:

- Kim E, Wang B, Sastry N, Masliah E, Nelson PT, Cai H, Liao F NEDD4-mediated HSF1 degradation underlies $\alpha$-synucleinopathy. Hum Mol Genet 2016; 25: 211-222.

- Wang B, Liu Y, Huang L, Chen J, Li JJ, Wang R, Kim E, Chen Y, Justicia C, Sakata K, Chen H, Planas A, Ostrom RS, Li W, Yang G, McDonald MP, Chen R, Heck DH, Liao F A CNS-permeable Hsp90 inhibitor rescues synaptic dysfunction and memory loss in APP- overexpressing Alzheimer's mouse model via an HSF1-mediated mechanism. Mol Psychiatry 2016 doi: 10.1038/mp.2016.104. [Epub ahead of print].

Minor revision in PLOS Genetics

- Kim E*, Nelson PT, Sakata K, Liao F* Bidirectional interplay of HSF1 degradation and UPR activation promotes tau hyperphosphorylation. (* : co-corresponding authors). 